THEORETICAL STUDIES OF RUPTURE PROCESSES IN GEOLOGICAL MATERIALS

\author{
J. W. Rudnicki \\ Department of Theoretical and Applied Mechanics \\ 216 Talbot Laboratory \\ University of Illinois \\ 104 S. Wright Street \\ Urbana, IL 61801
}

USGS CONTRACT NO. 14-08-0001-19146

Supported by the EARTHQUAKE HAZARDS REDUCTION PROGRAM

OPEN-FILE NO. 81-956

U.S. Geological Survey

OPEN FILE REPORT

This report was prepared under contract to the U.S. Geological Survey and has not been reviewed for conformity with USGS editorial standards and stratigraphic nomenclature. Opinions and conclusions expressed herein do not necessarily represent those of the USGS. Any use of trade names is for descriptive purposes only and does not imply endorsement by the USGS. 
THEORETICAL STUDIES OF RUPTURE PROCESSES

IN GEOLOGICAL MATERIALS

14-08-0001-19146

J. W. Rudnicki

Department of Theoretical and

Applied Mechanics

University of I1linois

Urbana, IL 61801

Summary of Accomplishments

During the year May 21, 1980 to May 20, 1981 we have accomplished some work in each of the three areas proposed for study: (i) interaction of slipping zones on faults; (ii) inception of shear rupture; and (iii) effects of coupling between deformation and pore fluid diffusion on rupture.

\section{Interaction of Slipping Zones}

Rudnicki and Kanamori (1981) have used existing quasi-static solutions for collinear shear cracks to examine quantitatively the effects of fault slip zone interaction on determinations of moment, stress drop, and static strain energy release. In addition, these calculations provide specific numerical examples of the general relations between moment and stress drop derived by Madariaga (JGR, 84, 2243-2250, 1979) and illustrate the extent to which small strong asperities or barriers can control the pattern of stress release.

As a preliminary to an examination of the dynamic effects of slip zone interaction, Rudnicki and Freund (1981) have reexamined the relationships among various expressions for the energy radiated by elastodynamic seismic sources. Both farfield representations and Kostrov's (Izv. Earth Physics, $1,23-40,1974)$ representation of radiated energy in terms of fault surface traction and particle velocity are examined. In particular, Kostrov's representation is arranged in various forms to reveal the source of radiated energy as the deviations of the fault surface tractions and particle velocities from the values that would occur during quasistatic fault motion between the same end states. Moreover, the excess of the static strain energy change over the work done by the fault surface tractions, called $W_{0}$ by Kanamori (JGR, 82, 2981-2987, 1977), is shown to be a good approximation to the radiated energy when fault propagation speed is near the Rayleigh wave velocity and the time rate of change of fault surface tractions is small. Because Kanamori has shown that for large earthquakes $W_{0}$ is approximately equal to the Gutenberg-Richter energy, one possible inference is that the conditions for which $W_{0}$ is approximately equal to the radiated energy are satisfied for large earthquakes. 
Our work in this area has concentrated on an extension of Rice's (JGR, $80,1531-1536,1975)$ analysis of the stability of dilatant hardening for a layer subjected to simple shear. In particular, we are examining the response in the case of a finite-size nonuniformity in the form of a sublayer already weakened, perhaps by past faulting. An analysis that linearizes the response of the weakened layer and that of the surrounding material about undrained (no change in fluid mass content) homogeneous deformation demonstrates the competing effects of increasing tectonic stress and pore fluid diffusion in aggravating and mitigating, respectively, the tendency for the deformation to concentrate in the weakened layer. A graduate student, G. L. Bowers, has been formulating a direct numerical solution of the problem. Numerical solutions demonstrate that dilatant hardening can delay failure by comparison with its occurrence in dry rock. Moreover, although Rice's (1975) analysis demonstrated that homogeneous undrained response became unstable, in the sense that local nonuniformities grow exponentially in time when the underlying drained response passes peak, the numerical results suggest that at higher rates of boundary displacement the material can be driven to a load maximum instability before significant growth of these nonuniformities can occur. However, for imposed strain-rates representative of tectonic processes $\left(\sim 10^{-14} \mathrm{sec}^{-1}\right)$ essentially drained conditions prevail until very near instability and dilatant hardening appears to be ineffective at increasing either the maximum stress or the maximum average strain of the layer. Consequently, the analysis does not yield evidence that dilatant hardening of an existing fault zone can transfer inelastic deformation and cracking to a much larger volume and cause accompanying precursory changes in material properties.

\section{Coupled-Deformation Diffusion Effects}

In addition to the above-mentioned work on dilatant hardening, which also involves coupled deformation diffusion effects, we have accomplished some additional work in this area.

Rudnicki (1980) has reviewed predictions for processes preparatory to earthquakes based on an inclusion model of earth faulting (Rudnicki, JGR, 82, 844-854, 1977; Rice and Rudnicki, JGR, 84, 2177-2193, 1979) for both dry and fluid-saturated rock masses. This review includes an interpretation of recent experimental results by Martin (GRL, $7,404-406,1980$ ) on the stabilization by dilatant hardening of failure in Westerly granite.

Rudnicki (1981) has also obtained a useful rearrangement of cleary's (Int. J. Solids Struc., 13,785-808, 1977) solution for the sudden application of a point force at the origin in a linear fluid-infiltrated porous elastic solid. In particular, the stress and displacement fields are demonstrated to comprise a time independent portion, the classical elastic solution based on the undrained (short-time) moduli, and a time dependent portion, the solution for a continuous fluid mass dipole. A corollary of this result is that the time dependent functions entering the point force solution can be obtained from a single function entering the displacement solution for a fluid mass source. Rudnicki has used singlular solutions constructed from the point force solution to reconstruct, by placing appropriate point singularities at the origin, the solution of Rice, Rudnicki, and Simons (Int. J. Solids Struic., 
14, 289-303, 1978) for the response of a fluid-infiltrated porous elastic solid containing a spherical cavity or inclusion. It is hoped that this technique may be used to obtain solutions for more complex geometries.

\section{Publications}

Rudnicki, J. W. and H. Kanamori, Effects of fault interaction on moment, stress drop, and strain energy release, J. Geophy. Res., 86, 1785-1793, 1981.

Rudnicki, J. W. and L. B. Freund, On energy radiation from seismic sources, BSSA, 71, 583-596, 1981.

Rudnicki, J. W., An inclusion model for processes preparatory to earthquake faulting. In Solid Earth Geophysics and Geotechnology, edited by $S$. Nemat-Nasser, Applied Mechanics Division, Vol. 42, Am. Soc. Mech. Engn., New York, pp. 39-52, 1980.

Rudnicki, J. W., On "Fundamental solutions for a fluid-saturated porous solid", by M. P. Cleary, Int. J. Solids Struc., 17, 855-857, 1981.

Rudnicki, J. W., Discussion of "On Finite Plastic Flows of Compressible Materials with Internal Friction", by S. Nemat-Nasser and A. Shokooh, Int. J. Solids Struc., to appear, 1981. 


\section{INTRODUCTION}

The purpose of these studies is to examine theoretical models for the rupture of geological materials in order to better understand the physical processes that precede and accompany earthquakes. Particular attention is given to processes that may make it possible to anticipate the occurrence of earthquake faulting. Specific studies in the following three areas are underway: (i) localization of deformation as a model for the inception of shear rupture; ( $i i)$ transient stabilization of rupture by coupling of deformation with diffusion of an infiltrating pore fluid; and (iii) interaction of slipping zones on faults and frictional surfaces. This report describes our work in each of these areas during the year May 20, 1980 to May 20, 1981. Some of the work in area ( $i i i$ ) involved the completion of two studies (Rudnicki and Kanamori (1981) and Rudnicki and Freund (1981)) that were begun in the interim between submission of the original proposal (February, 1979) and the beginning of funding (June, 1980). Onty a brief summary of this work is given in the text; a complete description is given in Appendix III and Appendix IV which reproduce the published manuscripts. Our main effort during the last six months of the contract has been in area ( $i$ ) where we have been investigating coupled deformation diffusion effects on localization of deformation. 
INCEPTION OF SHEAR RUPTURE

Because most earthquakes seem to occur on existing fault systems, many models of the earthquake processes assume that inelastic deformation is confined to the fault itself. However, many observations of changes in physical properties that have been suggested as possible precursors to earthquakes, for example, changes in resistivity, radon emission or seismic wave speed, imply cracking due to inelastic deformation over a region that is not confined to the vicinity of the fault. One possible means by which inelastic deformation can be transferred laterally from an existing fault zone has been suggested by Rice (1975): if ground water is present, dilatant hardening of the fault zone could cause adjacent regions to accommodate inelastic deformation. In order to investigate this possiblity, work in this area has focused on an extension of Rice's (1975) analysis of the stability of dilatant hardening for a fluid-saturated rock layer deformed in shear. Rice demonstrated that the dilatantly hardened response became unstable in the sense that infinitesimal nonuniformities grow exponentially in time when the underlying drained (constant pore fluid pressure) response satisfies the conditions for localization of deformation (Rudnicki and Rice, 1975). Our extension of this analysis considers the effect of a finite-size nonuniformity in the form of a sublayer with material properties that differ slightly from those of the surrounding material. In this case there are competing effects due to the rate of increase in applied shear (assumed to idealize tectonic loading) and the rate of pore fluid diffusion. The former tends to further concentrate the deformation in the initially weakened layer whereas the latter tends to transfer deformation from the initially weakened layer to adjacent material. In addition to the direct relevance of this problem to precursory processes, we hope that our analysis will make possible quantitative comparison of the theoretical 
predictions with experiments on dilatant hardening by Brace and Martin (1968) and by Martin (1980). Rudnicki (1980) has recently reviewed the results of the experiments by Martin (1980) in the context of predictions by Rice and Rudnicki (1979) on the stabilization of failure by dilatant hardening for an inclusion model of earthquake faulting (see Appendix I).

For simplicity, we have begun our analysis by studying the same geometric configuration analyzed by Rice (1975): one dimensional deformation of a layer (Figure 1). Because displacements depend only on $y$, the only non-zero strains are

$$
\gamma(y, t)=\partial u_{x}(y, t) / \partial y, \quad \varepsilon(y, t)=\partial u_{y}(y, t) / \partial y
$$

The stresses conjugate to the strains are $\tau$ and $\sigma$ (positive in compression), respectively, and, as shown by Rice (1975), equilibrium in the absence of body forces requires that these stresses be only a function of time $t$. We also adopt the constitutive rate 1 aw proposed by Rice (1975). For drained response, that is, constant pore fluid pressure response, this law is as follows:

$$
\begin{aligned}
& \dot{\gamma}=\dot{\tau} / G+(\dot{\tau}-\mu \dot{\sigma}) / H \\
& \dot{\varepsilon}=-\dot{\sigma} / M+B(\dot{\tau}-\mu \dot{\sigma}) / H
\end{aligned}
$$

$G$ and $M$ are elastic moduli and hence the first term in each equation is the elastic strain rate. The second term in each equation is the inelastic contribution and these terms are dropped for deformation increments that tend to cause elastic unloading, that is, deformation increments for which $\dot{\tau}<\mu \dot{\sigma}$. The parameter $\mu$ is a friction coefficient expressing the inhibiting effect of hydrostatic compression on inelastic deformation, $\beta$ is a dilatancy factor equal to the ratio of inelastic increments of volume strain to shear strain, and $H$ is an inelastic modulus termed hardening if positive or softening if negative. The modulus $H$ is related to the slope of the $\tau$ versus $\gamma$ curve at constant $\sigma$ by 


$$
H=\frac{d \tau}{d \gamma}\left(1+\frac{d \tau}{d \gamma}\right)^{-1} \quad(\sigma=\text { constant })
$$

To describe the response during arbitrary variations in the pore fluid pressure, one simply replaces the stress $\sigma$ by the effective stress $\sigma_{e}=\sigma-p$, where $p$ is the pore fluid pressure. This form of the effective stress is appropriate for inelastic deformation arising from frictional sliding on fissure surfaces and microcracking from the tips of sharp flaws (Rice, 1977) and for elastic deformation if the solid and fluid constituents are incompressible. Here we will specialize to the case of incompressible constituents in order to simplify the notation although the case of compressible constituents can be treated in the same manner. Thus, replacing $\sigma$ by $\sigma-p$ in (2) yields

$$
\begin{aligned}
& \dot{\gamma}=\dot{\tau} / G+[\dot{\tau}-\mu(\dot{\sigma}-\dot{p})] / H \\
& \dot{\varepsilon}=-(\dot{\sigma}-\dot{p}) / M+B[\dot{\tau}-\mu(\dot{\sigma}-\dot{p})] / H .
\end{aligned}
$$

An additional constitutive equation is Darcy's law which, in the absence of body forces, can be written as

$$
q=-\rho \kappa \partial p / \partial y
$$

where $\rho$ is the density of fluid mass, $k$ is a permeability, and $q$ is the mass flow rate per unit area in the $y$ direction. As noted earlier, the equilibrium equations simply require that $\tau$ and $\sigma$ be functions only of time. The remaining relevant field equation is fluid mass conservation. In the case of incompressible constituents, fluid mass conservation requires that

$$
\frac{\partial q}{\partial y}+\rho \frac{\partial \varepsilon}{\partial t}=0
$$

Substituting Darcy's law (6) into this equation yields

$$
\frac{\partial}{\partial y}\left(\rho \kappa \frac{\partial p}{\partial y}\right)=\rho \frac{\partial \varepsilon}{\partial t}
$$


or, for constant $\rho$ and $k$,

$$
\kappa \frac{\partial^{2} p}{\partial y^{2}}=\frac{\partial \varepsilon}{\partial t}
$$

For undrained (no change in fluid mass content) homogeneous deformation, $\partial \varepsilon / \partial t=0$ and, hence, solving (5) for $\dot{p}$ yields

$$
\dot{p}=\frac{-\beta M \dot{\tau}}{H+\mu \beta M}
$$

where, for convenience, $\sigma(t)$ has been assumed constant. Substituting (9) into (4) reveals that the effective value of the hardening modulus has been augmented form $H$ to $H+\mu \beta M$ for undrained response. This effective stiffening of the response is referred to as dilatant hardening and occurs as a result of' the tendency of dilatancy to decrease the pore fluid pressure (9). If the decrease of pore fluid pressure occurs faster than it can be alleviated by fluid mass diffusion, the effective compressive stress increases and this increase inhibits further inelastic response.

Our studies have considered the response to the equations described above when there is an initial nonuniformity in the form of a sublayer having inelastic material properties differing slightly from those of the remainder of the material. One approach examines perturbations about the undrained homogeneous response. That is, the layer is assumed to be deformed to some point in homogeneous undrained fashion and then the subsequent response is considered when the layer is divided into two sublayers with slightly different hardening moduli. The response in each sublayer is then linearized. This analysis has been described in our Semi-Annual Technical Report No. 1 (January 9, 1981) and will not be repeated here.

Numerical Approach

A second approach to this problem is a direct numerical solution by a 
graduate student, G. L. Bowers, as a portion of his Ph.D. thesis. The geometry is shown in Figure 1 where, because of symmetry, only the upper half is drawn. The equations can be recast in a form more suitable for numerical analysis by substituting (5) into (8) and using (4) to eliminate $\dot{\tau}$. The result is

$$
M_{K} \frac{(H+G)}{H+G+\mu \beta M} \frac{\partial^{2} p}{\partial y^{2}}-\frac{\partial p}{\partial t}=\frac{M G}{H+G+\mu \beta M} \frac{\partial \gamma}{\partial t}
$$

where, for convenience, it has been assumed that $\dot{\sigma}=0$. Because the hardening modulus $H$ depends on the strain, (10) must be solved simultaneously with the relation (4) for $\dot{\tau}$ subject to the equilibrium condition that

$$
\frac{\partial \tau}{\partial y}=0 \rightarrow \tau=\tau(t)
$$

Note that the coefficient of the first term in (10) is a diffusivity that depends on the deformation through $H$. The quantity $c=M_{k}$ is the diffusivity appropriate for elastic deformation $(H \rightarrow \infty)$. By choosing the length scale as the half-layer thickness $L$ and the time scale as $L / \dot{U}$, the inverse of the average shear strain rate, (10) can be rewritten as

$$
\left(\frac{C}{\dot{U} L}\right) \frac{H+G}{H+G+\mu \beta M} \frac{\partial^{2} p}{\partial(y / L)^{2}}-\frac{\partial p}{\partial(\dot{U} t / L)}=\frac{\beta M G}{H+G+\mu \beta M}-\frac{\partial \gamma}{\partial(\dot{U} t / L)}
$$

This form of the equation reveals the significance of the nondimensional parameter

$$
\eta=\frac{\dot{U} L}{c}
$$

which is the product of the average shear strain rate $\dot{U} / L$ and a diffusion time for elastic deformation $t_{D}=L^{2} / c$, and, hence, is a measure of the relative time scales associated with boundary straining and pore fluid diffusion. For rapid straining $n$ will be large, the coefficient of the first term in (12) will be small (unless $H$ is negative and so large in magnitude 
that it approaches $\mu \beta M$ ) and the effects of diffusion will be relatively insignificant. Conversely, for smaller values of $\eta$ diffusion will be more important.

Symmetry requires that the boundary conditions on $y=0$ be

$$
u_{x}=0 \text { and } q=-\kappa \frac{\partial p}{\partial y}=0 \text {. }
$$

At $y=L$ the boundary conditions are

$$
q=0, \quad \dot{u}_{x}=\dot{U}
$$

In addition, the displacements, pore fluid pressure and fluid mass flux must be continuous at $y=\ell_{1}$ where the material properties were assumed to change. (The stresses are already required by equilibrium to be continuous.) The initial conditions are

$$
p=0, \quad u_{x}=0, \quad \text { and } \tau=0, \text { at } t=0 .
$$

Solution Method

Numerical solution was obtained using the second order-accurate (in space and time) Crank-Nicholson (C-N) version of the finite difference method. Although the $C-N$ method determines the solution at the new time $t_{n+1}$, it requires that the coefficients be evaluated at the time $t_{n+1 / 2}$, which is halfway between $t_{n+1}$ and the current time, $t_{n}$. Thus, the method is implicit, and the following iterative scheme was employed (Van Rosenberg, 1976):

1) Using the known values of the coefficients at $t_{n}$, the time was incremented by $\Delta t / 2$, (half the normal time step size), and the equations were solved for first estimates of $u_{x}$ and $\sigma_{e}$ at $t_{n+1 / 2}$.

2) The results of Step 1 were used to evaluate the coefficients at $t_{n+1 / 2}$, and solutions were computed at the time $t_{n+1}$.

3) By averaging the results at $t_{n+1}$ and $t_{n}$, new estimates were foünd 
for $u_{x}$ and $\sigma_{e}$ at $t_{n+1 / 2}$, and these were used to recalculate the coefficients.

4) Using the coefficients from Step 3, the equations were again solved at the time $t_{n+1}$, and Steps 3 and 4 were repeated until the results from two consecutive iterations converged to within an allowable error. Normally, it was found that convergence occurred after onty one iteration (of Steps 3 and 4).

The C-N method is unconditionally stable for all choices of time step sizes and nodal spacings. However, it is known to be oscillatory for the homogeneous diffusion equation if the quantity $c \Delta t / \Delta y^{2}$ is large and pressure gradients are steep; where $c$ is the diffusivity, $\Delta t$ the time step, and $\Delta y$ the distance between nodes (Desai and Johnson, 1973). Because, as the weakened zone dilates, local flows could induce steepening pressure gradients, and the solution is very sensitive to perturbations in pressure near peak stress, the computer program was written so that the time step size was decreased as the stress approached peak.

Results

For the purposes of calculation, the shear stress vs. shear strain curve, at constant effective normal stress, was assumed to be the same as that used by Rice (1979) and by Rice and Rudnicki (1979): a parabola connected smoothly at the elastic limit $\tau_{0}$ to a linear segment of slope $G$. This relation is shown in Figure 2 and is described by

$$
\begin{aligned}
& \tau=G_{\gamma}, \tau<\tau_{0} \\
& \tau=\tau_{p}-\frac{G}{2 \lambda}\left(\gamma-\gamma_{p}\right)^{2}, \quad \tau>\tau_{0}
\end{aligned}
$$

where $\gamma_{p}$ is the strain at peak stress $\tau_{p}$ and $\lambda$ is the difference between 
$\gamma_{p}$ and the strain at the elastic limit. The stress at the elastic limit is related to that at peak by

$$
\tau_{0}=\tau_{p}-\frac{1}{2} G \lambda
$$

The peak stress of the stronger sublayer $\left(l_{1}<y \leq L\right)$ was assumed to have a value $\tau_{\mathrm{p} 2}=1.25 \mathrm{~kb}$ whereas that of the weaker sublayer $\left(0 \leq \dot{y} \leq \ell_{\gamma}\right)$ was $\tau_{p l}=0.97 \mathrm{~kb}$. For both layers, $\lambda=2.5 \times 10^{-3}$ and $G=200 \mathrm{~kb}$. Consequently, $\tau_{01}=0.72 \mathrm{~kb}$ for the weaker sublayer and $\tau_{02}=1.00 \mathrm{~kb}$ for the stronger sublayer. Other material parameters were assumed to be the same for both sub1ayers:

$$
\mu=0.6, \quad B=0.3, \quad M=534 \mathrm{~kb}, \quad c=0.1 \mathrm{~m}^{2} / \mathrm{sec} .
$$

In all the calculations, the thickness of the initially weaker sublayer was one-tenth the thickness of the initially stronger sublayer.

Calculations were made for values of (U் $/ \mathrm{C})$ from 1.2 to $1.2 \times 10^{-4}$. The results are shown in Figures 3 to 5 . Figure 3 shows the strain history of the first point in the layer to reach instability as a function of the average strain $U / L$ for three values of $\dot{U} L / c$. Also shown for comparison is the strain history for dry rock, where the curve is drawn for an infinitesimal weakened sublayer thickness. The corresponding stress strain curves are shown in Figure 4. Figure 5 shows the distribution of the pore fluid pressure decrease in the weaker sublayer immediately before instability, as identified from Figure 3. Values of the nondimensional time $\dot{U} t_{\max } / L$ at which instability occurred are listed in Table I. It is evident from Figure 5 that the weakest point of the layer occurs at the interface between the sublayers where $-p$ is lowest (lowest effective stress). This point was used to plot Figures 3 and 4. 
For the highest loading rate $\dot{U} \mathrm{~L} / \mathrm{c}=1.2$, Figure 3 indicates that almost half of the weakened sublayer is at undrained conditions. As expected, the magnitude of the pore fluid pressure decrease diminishes as the interface between the sublayers is approached and drops rapidly in the stronger sublayer. As $\dot{U} L / C$ decreases, diffusion becomes more effective in maintaining uniform pressure distributions of lower magnitude. Because the total fluid mass of the layer is finite, the average fluid pressure must decrease as dilation occurs. Consequently, it is not possible to achieve perfectly drained conditions $(\dot{p}=0)$ even at very 'slow rates of deformation.

Table I tabulates some results from Figures 3 and 4 . Shown is a comparison between the tangent modulus $H_{\tan }=H /(7+H / G)$ at instability for the weakest point in the layer and the tangent modulus $H_{\tan }^{d}$ for the underlying drained response at the current value of the effective stress. At the higher rates of deformation, instability occurred when the actual stress strain curve was at its peak $\left(H_{\tan }=0\right)$. However, at slower rates instability occurred when $H_{\text {tan }}$ was positive, but the underlying drained response was at its peak, $H_{\tan }^{\mathrm{d}}=0$. Recall that Rice's (1975) linearized perturbation analysis indicated that perturbations would grow exponentially in time when the underlying drained response exceeds peak. However, the results here suggest that at higher deformation rates, the material is driven to a load maximum instability before such perturbations can cause failure. At slower rates rapid growth of perturbations triggered by $H_{\tan }^{d}=0$ evidently cause instability at this point. Also shown in Table I are the maximum average strain of the layer $\left(U_{\max } / L\right)$ and the maximum shear stress attained.

Table II summarizes the influence of coupled deformation - diffusion effects on the strength of the material and on precursory deformation. The 
ratio $U_{\max } / U_{p}^{d}$, where $U_{p}^{d}$ is the surface dipslacement at which dry rock reaches peak stress, indicates that diffusion effects increase the total average strain that can be achieved before instability. The third column indicates amount by which the maximum stress will exceed the peak stress in the weaker sublayer under drained conditions and the last two columns demonstrate the extent to which instability is delayed.

The precursor time calculations for the two slowest deformation rates involved a revised value for the dry rock time $t_{p}^{D}$. As was discussed earlier, because the second layer of material was not an infinite source of fluid made it impossible for slow deformation rates to ever achieve drained conditions $\left(\sigma_{e}=\right.$ constant $\left.=0\right)$. This limitation of the model caused additional, artificial, dilatant hardening effects due to the mean pressure decreasing. Consequently, the pressure at the $y=L$ was used as a measure of how much the effective stress had been artifically enhanced. Hence, instead of comparing the results for the two slowest deformation rates with dry rock, for which $\sigma_{e}=0$, they were compared with drained rock, but at an effective stress equal to the effective stress at $y=L$.

Concluding Discussion

The results presented here indicate that dilatant hardening can delay failure by comparison to its occurrence in dry rock. Moreover, although Rice's (1975) analysis demonstrated that homogeneous undrained response became unstable, in the sense that local nonuniformities grow exponentially in time when the underlying drained response passed peak, the results here suggest that at higher rates of deformation the material can be driven to a load maximum instability before significant growth of these nonuniformities occur. However, for a representative tectonic strain rate $\frac{\dot{U}}{L}=10^{-14} / \mathrm{sec}$ (corresponding to 
0.1 bar/year using $G=200 \mathrm{~kb}), L=5 \mathrm{~km}$ and $c=0.1 \mathrm{~m}^{2} / \mathrm{sec}, \dot{U} L / c=2.5 \times 10^{-6}$. For this rate, the results here suggest essentially drained conditions prevail and instability is controlled by the drained response. Moreover, at such slow rates dilatant hardening appears to be ineffective at increasing either the maximum stress or average strain of the layer. Consequentiy, it is not anicicipated that precursory effects due to widespread cracking or dilatancy would be observed.

Even for this simple geometry, direct numerical solution has proven to be quite expensive. This is the result of having to choose small time steps in order to resolve instability even for small values of $\dot{U} L / c$. Consequently, we are currently pursuing an approach which, although it still requires numerical solution, simplifies the diffusion aspects of the problem and, as a result, will be more economical. A brief description of this approach is given in our SemiAnnual Technical Report, and we intend to investigate this approach further during the next year.

Table I: Data for Layer at the Onset of Instability

\begin{tabular}{lcccc}
\hline UL/C & $\begin{array}{c}\mathrm{H}_{\tan } / \mathrm{G} \text { at } \\
\text { Failure }\end{array}$ & $\begin{array}{c}\mathrm{H}_{\tan }^{\mathrm{d}} / \mathrm{G} \text { at } \\
\text { Failure }\end{array}$ & $\begin{array}{c}\mathrm{U}_{\max } / \mathrm{L} \\
\left(10^{-3}\right)\end{array}$ & $\tau_{\max }$ \\
\hline 1.2 & 0 & -0.074 & 5.133 & 1.013 \\
$1.2 \times 10^{-1}$ & 0 & -0.053 & 5.127 & 1.010 \\
$1.2 \times 10^{-2}$ & 0.028 & 0 & 5.089 & 1.000 \\
$1.2 \times 10^{-3}$ & 0.023 & 0 & 5.036 & 0.987 \\
$1.2 \times 10^{-4}$ & 0.014 & 0 & 5.017 & 0.982 \\
\hline
\end{tabular}


Table II: Comparison between Dry Rock and Saturated Rock for Different Rates of Deformation

\begin{tabular}{ccccc}
\hline$\dot{U} L / c$ & $U_{\max } / U_{p}^{d}$ & $\tau_{\max } / \tau_{p}^{d}$ & $\begin{array}{c}\left(t_{\max }-t_{p}^{D}\right) \\
\left(L^{2} / c\right)\end{array}$ & $\begin{array}{c}\left(t_{\max }-t_{p}^{d}\right) / t_{p}^{d} \\
(\%)\end{array}$ \\
\hline 1.2 & 1.034 & 1.044 & $1.4 \times 10^{-4}$ & 3.412 \\
$1.2 \times 10^{-1}$ & 1.033 & 1.042 & $1.4 \times 10^{-3}$ & 3.280 \\
$1.2 \times 10^{-2}$ & 1.025 & 1.031 & $1.02 \times 10^{-2}$ & 2.496 \\
$1.2 \times 10^{-3}$ & 1.009 & 1.012 & $3.69 \times 10^{-2^{*}}$ & $0.894^{*}$ \\
$1.2 \times 10^{-4}$ & 1.001 & 1.002 & $4.89 \times 10^{-2^{*}}$ & $0.112^{*}$ \\
\hline
\end{tabular}

*Note: Compensation was made for additional dilatant hardening effects which were caused by the limitations of the model (see text). 
COUPLED DEFORMATION - PORE FLUID DIFFUSION EFFECTS

In addition to our work described in the previous section, which also involves coupled deformation diffusion effects, we have been exploring the use of singular solutions for fluid-infiltrated elastic solids to solve boundary value problems. The goal of these studies is to obtain solutions analogous to that of Rice, Rudnicki and Simons (1978) for spherical cavities or inclusions for other geometries, in particular, axisymmetric ellipsoids. This would make possible a study of the effects of geometry on the earthquake precursory processes examined by Rice and Rudnicki (1979) for the spherical inclusions.

The basis for this work is Rudnicki's (1981) rearrangement of Cleary's (1977) fundamental solution for linear fluid-infiltrated elastic solids. This rearrangement and the correction of a minor algebraic error by cleary is described in Appendix II. The main result is that the response to sudden application of a point force can be written as the sum of the time-independent classical elasticity solution based on the undrained (short-time) elastic constants and the solution to a continuous fluid mass dipole. More specifically, the displacement $u_{i}$ and stress $\sigma_{i j}$ due to sudden application of a point force $P_{\alpha}$ at the origin are given by (12) and (13) (Noting (32) - (36)) of Appendix II.

This rearrangement of the fundamental solution may be useful in solving certain boundary value problems. In order to investigate this possibility, we have attempted to reconstruct the solutions of Rice and Cleary (1976) and of Rice, Rudnicki, and Simons (1978) for loading of a spherical cavity by means of a singularity technique: appropriate singularities derived from the point force solution are placed at the center of the cavity and their strengths 
are chosen to satisfy the boundary conditions. This technique is analogous to that used by Timoshenko and Goodier (1970, art. 137) to solve the equivalent elasticity problem of a spherical cavity loaded by remote tension (The solution by Rice et a1. (1978) is analogous to the approach of Love (1944, article 173)). Although the procedure is straightforward, the detailed algebra is tedious. We will outline the solution for a spherical cavity loaded by radial tractions and pore fluid pressure at the cavity boundary. This problem has, of course, been solved elsewhere (see, for example, Rice and Cleary (1976)) but, nevertheless, it will suffice to illustrate the technique.

The singular solution needed to solve the elasticity problem of radial loading of a spherical cavity is the center of dilation. If the displacements due to a point force $P_{\alpha}$ at $\underset{\sim}{x}=\underset{\sim}{x}$ are given by

$$
u_{\alpha}(\underset{\sim}{x})=P_{\beta} g_{\alpha \beta}\left(\underset{\sim}{x}-\underline{\sim}^{\prime}\right),
$$

the displacements due to a center of dilation are given by

$$
u_{\alpha}(\underset{\sim}{x})=-m \frac{\partial g_{\alpha \beta}}{\partial x_{\beta}}\left(x-x^{\prime}\right) .
$$

where $m$ is the strength of the singularity. Consequently, we begin with a center of dilation in a linear fluid-infiltrated elastic solid. The stress $\sigma_{\alpha \beta}$ and pore fluid pressure $p$ due to a center-of-dilation of magnitude $m$ are

$$
\begin{aligned}
\sigma_{\alpha \beta}(\underset{\sim}{x}, t)= & \frac{m}{2 \pi r^{3}} \frac{\mu}{\left(\lambda_{u}+2 \mu\right)}\left[\delta_{\alpha \beta}-\frac{3 x_{\alpha} x_{B}}{r^{2}}\right] \\
& +\frac{\left(\lambda_{u}-\lambda\right) \mu}{\left(\lambda_{u}+2 \mu\right)(\lambda+2 \mu)} \frac{m}{2 \pi r^{3}} \Gamma_{\alpha \beta}(\underset{\sim}{x}, \xi) \\
p(x, t)= & -\frac{\left(\lambda_{u}-\lambda\right)}{\zeta\left(\lambda_{u}+2 \mu\right)} \frac{m}{2 \pi r^{3}} \xi h_{j}
\end{aligned}
$$


where

$$
\Gamma_{\alpha \beta}(\underset{\sim}{x}, \xi)=\delta_{\alpha \beta}\left[1-\left(h_{1}-\xi h_{1}^{\prime}\right)\right]-\frac{3 x_{\alpha} x_{\beta}}{r^{2}}\left[1-\left(h_{1}-\frac{1}{3} \xi h_{j}^{\prime}\right)\right]
$$

and the notation otherwise is the same as that in Appendix II. A second, purely diffusive, singularity is also needed. By noting the correspondence between elastic singular solutions and pure diffusion singular solutions, the appropriate singularity is found to be an instantaneous source of fluid mass. The stress and pore fluid pressure fields due to instantaneous injection of an amount $Q$ of fluid mass at the origin $t=0$ are given by (20) and (21) of Appendix II. For our purpose here, it is more convenient to use this solution minus their long-time values. The results are

$$
\begin{aligned}
& \sigma_{\alpha \beta}=-\frac{Q}{\rho_{0}} \frac{\mu\left(\lambda_{u}-\lambda\right)}{2 \pi r^{3} \zeta\left(\lambda_{u}+2 \mu\right)} \Gamma_{\alpha \beta}(\underset{\sim}{x}, \xi) \\
& p=\frac{\left(\lambda_{u}-\lambda\right)(\lambda+2 \mu)}{\zeta^{2}\left(\lambda_{u}+2 \mu\right)} \frac{Q}{\rho_{0}} \frac{1}{4 \pi r^{3}} \xi h_{j}^{\prime}
\end{aligned}
$$

where, again, the notation follows Appendix II.

The solutions due to time-dependent source strengths $m(t)$ and $Q(t)$ can easily be constructed by superposition. The results are

$$
\begin{aligned}
& \sigma_{\alpha \beta}(\underset{\sim}{x}, t)= \frac{\mu}{\left(\lambda_{u}+2 \mu\right)} \frac{m(t)}{2 \pi r^{3}} *\left[\delta_{\alpha \beta}-\frac{3 x_{\alpha} x_{\beta}}{r^{2}}\right] \\
&+\frac{\left(\lambda_{u}-\lambda\right)}{\left(\lambda_{u}+2 \mu\right)} \mu \frac{F(t)}{2 \pi r^{3}} * \Gamma_{\alpha \beta}(\underset{\sim}{x}, \xi) \\
& p(\underset{\sim}{x}, t)=-\frac{\left(\lambda_{u}-\lambda\right)(\lambda+2 \mu)}{\zeta\left(\lambda_{u}+2 \mu\right)} \frac{F(t)}{4 \pi r^{3}} * \xi h_{1}^{\prime}
\end{aligned}
$$

where $F(t)=\frac{m(t)}{(\lambda+2 \mu)}-\frac{Q(t)}{\zeta \rho_{0}}$ 
and * denotes the convolution operation defined by

$$
f(t) * g(t)=\int_{0}^{t} f\left(t-t^{\prime}\right) g\left(t^{\prime}\right) d t^{\prime}
$$

The time-dependent strengths must be chosen to satisfy the following boundary conditions on the surface $r=a$ :

$$
\begin{aligned}
& p(r=a, t)=\underline{p}_{0} \\
& \frac{x_{\alpha}}{a} \sigma_{\alpha \beta}=-\sigma_{0} \frac{x_{\beta}}{a}
\end{aligned}
$$

where $\underline{P}_{0}$ and $\sigma_{0}$ are constants. Substituting (22) and (23) into (25a) and (25b) yield

$$
\begin{aligned}
-\sigma_{0} & =\frac{-1}{\pi a^{3}} \frac{\mu}{(\lambda+2 \mu)} \int_{0}^{t} m\left(t^{\prime}\right) H\left(t-t^{\prime}\right) d t^{\prime} \\
& -\frac{\mu\left(\lambda_{u}-\lambda\right)}{\left(\lambda_{u}+2 \mu\right)} \frac{1}{\pi a^{3}} F(t) *\left[1-h_{1}(a / \sqrt{c t})\right] \\
\underline{p}_{0}= & \frac{\left(\lambda_{u}-\lambda\right)(\lambda+2 \mu)}{\zeta\left(\lambda_{u}+2 \mu\right)} \frac{1}{4 \pi a^{3}} F(t) *(a \sqrt{c t}) h_{1}^{\prime}(a / \sqrt{c t})
\end{aligned}
$$

where $H(t)$ is the unit step function. These are simple integral equations of convolution type for $m(t)$ and $Q(t)$. Solution can be accomplished by using the Laplace transform

$$
\hat{f}(s)=L\{f(t)\}=\int_{0}^{\infty} e^{-s t} f(t) d t
$$

and noting the property

$$
L\{f * g\}=\hat{f} \hat{g} .
$$

The transforms of the functions $h_{1}(\xi)$ and $\xi h_{j}(\xi)$ are easily shown to be

$$
\hat{h}_{1}(s)=\frac{1}{s}\left[1-e^{-r \sqrt{s / c}}(1+r \sqrt{s / c})\right]
$$


and

$$
L\left\{\xi h_{j}(\xi)\right\}=\frac{r^{2}}{c} e^{-r \sqrt{s / c}} .
$$

Thus, the transformed boundary conditions become

$$
\begin{aligned}
\sigma_{0} \pi a^{3} s^{-1} & =\frac{\mu}{\left(\lambda_{u}+2 \mu\right)} \hat{m}(s) s^{-1} \\
& +\frac{\left(\lambda_{u}-\lambda\right)}{\left(\lambda_{u}+2 \mu\right)} F(s) e^{-a \sqrt{s / c}} s^{-1}(1+a \sqrt{s / c}) \\
4 \pi a^{3} \underline{p}_{0} s^{-1} & =-\frac{\left(\lambda_{u}-\lambda\right)}{\zeta\left(\lambda_{u}+2 \mu\right)}(\lambda+2 \mu) F(s) \frac{a^{2}}{c} e^{-a \sqrt{s / c}}
\end{aligned}
$$

Solving for $\hat{m}(s)$ and $\hat{F}(s)$ yields

$$
\begin{gathered}
\hat{F}(s)=-p_{0} \frac{\zeta\left(\lambda_{u}+2 \mu\right)}{\left(\lambda_{u}-\lambda\right)(\lambda+2 \mu)} 4 \pi a^{3} \frac{e^{a \sqrt{s / c}}}{s a^{2} / c} \\
\hat{m}(s)=a^{3} \frac{\left(\lambda_{u}+2 \mu\right)}{\mu} \sigma_{0}+\underline{p}_{0} \frac{\left(\lambda_{u}+2 \mu\right)}{(\lambda+2 \mu)} 4 \pi a^{3} \frac{(1+a \sqrt{s / c})}{\mathrm{sa}^{2} / \mathrm{c}}
\end{gathered}
$$

Note that the inversion of $\hat{F}(s)$ will not converge (because of the exponential $\mathrm{e}^{\mathrm{a} \sqrt{\mathrm{s} / \mathrm{c}}}$ ). Nevertheless, taking the transforms of (22) and (23), substituting from (28) and (29) and inverting does yield the solution given by Rice and Cleary (1976).

This procedure has been used to reconstruct the solution of Rice et al. (1978) for shear loading of a spherical cavity, but the algebra is more complicated. One difficulty in applying this technique to other geometries is that the appropriate position of the singularities is not known a priori. Nevertheless, for axisymmetric ellipsoids, it seems that a solution can be obtained by distributing the singularities along the center line. 
INTERACTION OF FAULT SLIP ZONES

A full description of our work in this area is given in Appendixes III and IV which reproduce published manuscripts. Appendix III (Rudnicki and Kanamori, 1981) describes the use of solutions for collinear shear cracks to examine quantitatively the effects of slip zone interactions on seismic moment, static stress drop and strain energy release. These calculations were purely quasistatic, but an effort to obtain similar results for dynamic conditions led to an investigation of the concept of radiated energy. This investigation is described in Appendix IV (Rudnicki and Freund, 1981). Various expressions, in terms of far-field quantities and fault surface tractions and particle velocities, are given for the radiated energy. In addition, the relationship of radiated energy to other energies involved in earthquake faulting is clarified. 


\section{BIBLIOGRAPHY}

Brace, W. F. and R. J. Martin, A test of the law of effective stress for crystalline rocks of low porosity, Int. J. Rock Mech. Min. Sci., $\underline{5}$, 415-426, 1968.

Cleary, M. P., Fundamental solutions for a fluid-saturated porous solid, Int. J. Solids Structures, 13, 785-808, 1977.

Desai, C. S. and L. D. Johnson, Evaluation of some numerical schemes for consolidation, Int. J. Num. Method Engrg. 7, 243-254, 1973.

Love, A. E. H., A Treatise on the Mathematical Theory of Elasticity, 4th edition, Dover, New York, 641 pp., 1941.

Martin, R. J., Pore pressure stabilization of failure in Westerly granite, Geophys. Res. Letters, 7, 404-406, 1980.

Rice, J. R., On the stability of dilatant hardening for saturated rock masses, J.G.R., 80, 1531-1536, 1975.

Rice, J. R., Pore pressure effects in inelastic constitutive formulations for fissured rock masses, in Advances in Civil Engineering through Engineering Mechanics, ASCE, New York, 1977, pp. 360-363.

Rice, J. R., Theory of precursory processes in the inception of earthquake rupture, Gerländs Beitr. Geophys., 88, 91-127, 1979.

Rice, J. R. and M. P. Cleary, Some basic stress-diffusion solutions for fluid-saturated porous media with compressible constituents, Rev. Geophys. Space Physics, 14, 227-241, 1976.

Rice, J. R., J. W. Rudnicki, and D. A. Simons, Deformation of spherical cavities and inclusions in fluid-infiltrated elastic materials, Int. J. Solids Struct., 14, 289-303, 1978.

Rice, J. R. and J. W. Rudnicki, Earthquake precursory effects due to pore fluid stabilization of a weakening fault zone, J.G.R., 84, 2177-2193, 1979.

Rudnicki, J. W., An inclusion model for processes preparatory to earthquake faulting, in Solid Earth Geophysics and Geotechnology, edited by S. Nemat-Nasser, pp. 39-52, Applied Mechanics Division, Vol. 42, American Society of Mechanical Engineers, New York, 1980.

Rudnicki, J. W., On "Fundamental Solutions for a Fluid-Saturated Porous Solids" by M. P. Cleary, Int. J. Solids Struc., 17, 855-857, 1981.

Rudnicki, J.W. and J. R. Rice, Conditions for the localization of deformation in pressure-sensitive dilatant materials, J. Mech. Phys. Solids, 23, . $371-394,1975$. 
Rudnicki, J. W. and L. B. Freund, on energy radiation from seismic sources, BSSA, 71, 583-596, 1981.

Rudnicki, J. W. and H. Kanamori, Effects of fault interaction on moment, stress drop, and strain energy release, J.G.R., 86, 1785-1793, 1981.

Timoshenko, S. P. and J. N. Goodier, Theory of Elasticity, 3rd edition, McGrawHi11, New York, 567 pp. 1951.

Van Rosenberg, D. U., Methods for the Numerical Solution of Partial Differential Equations, American Elsevier, New York, 1969. 


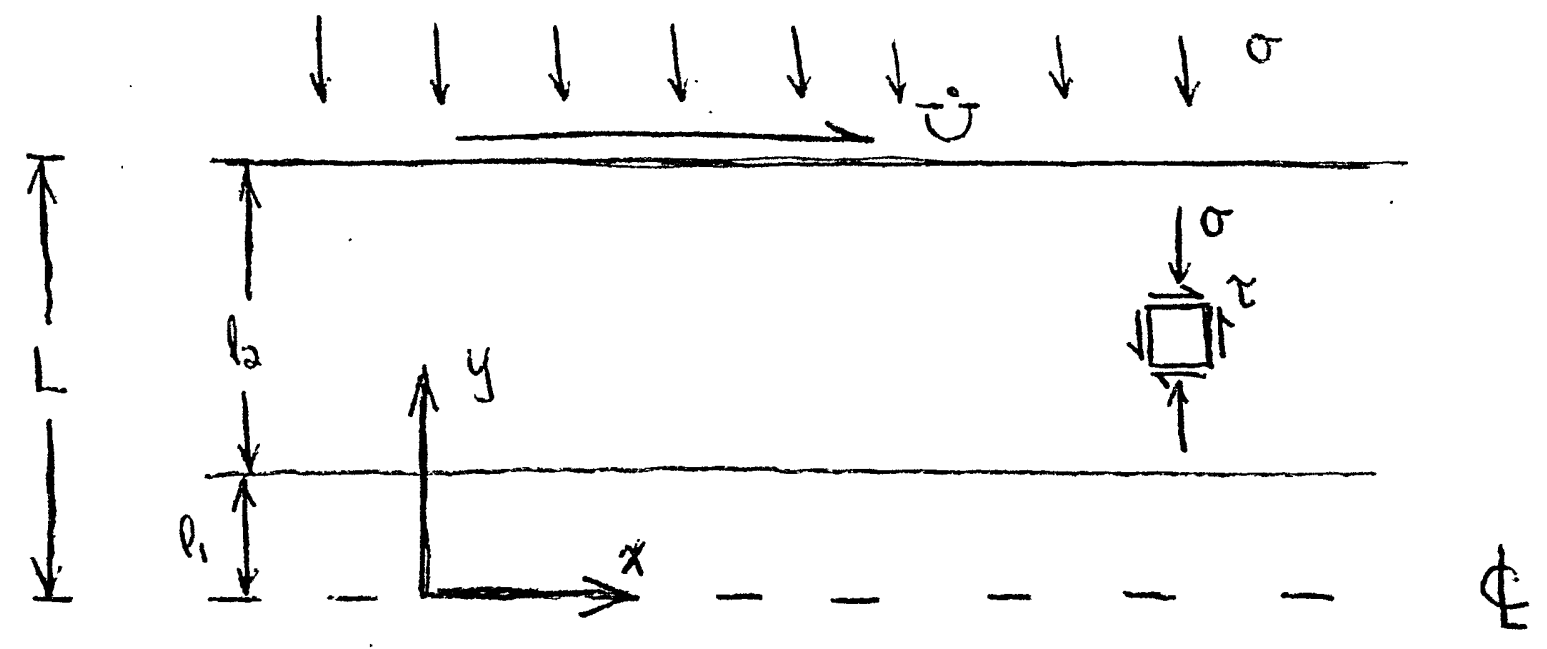

Figure 1

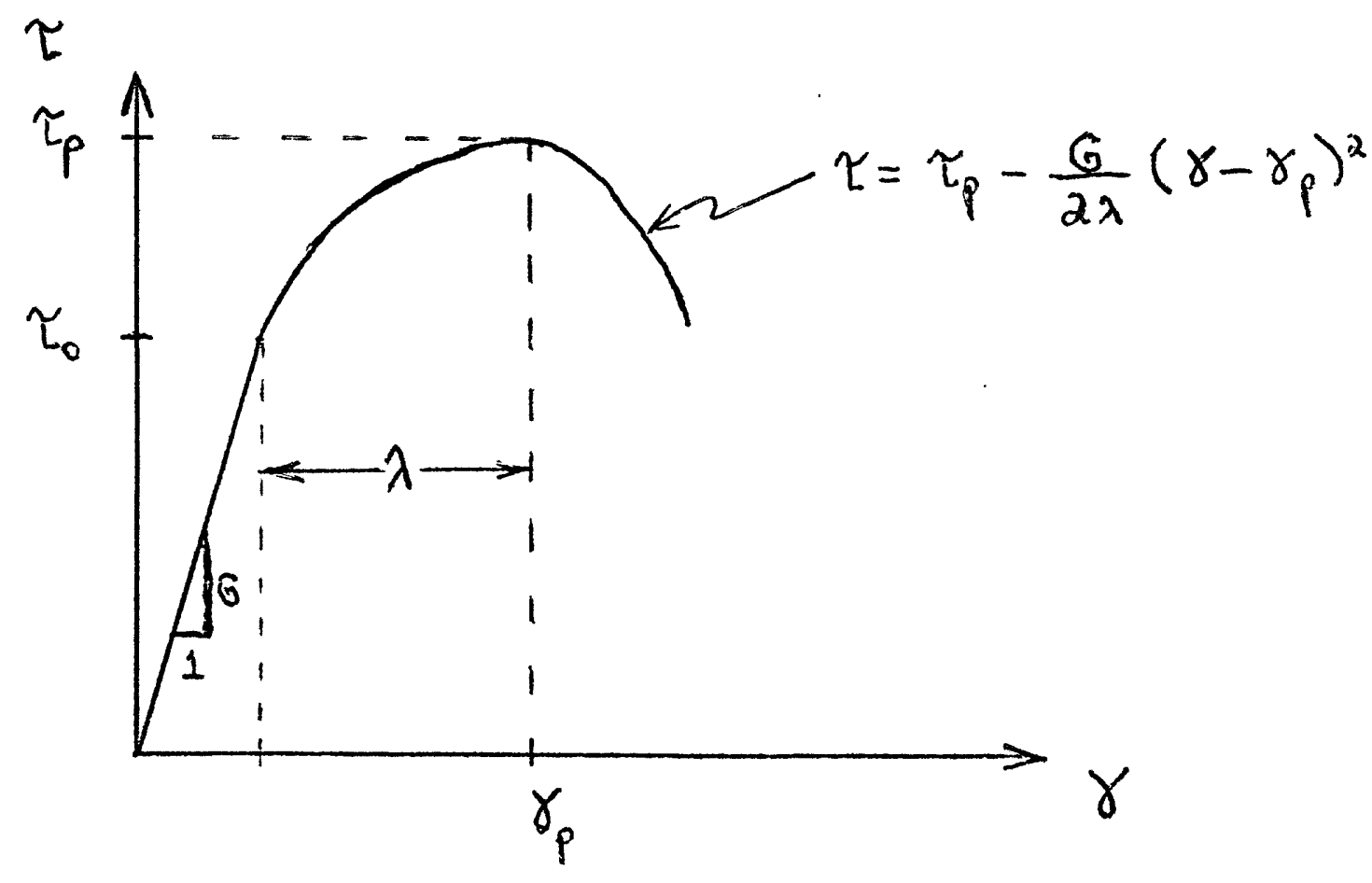

Figure 2 


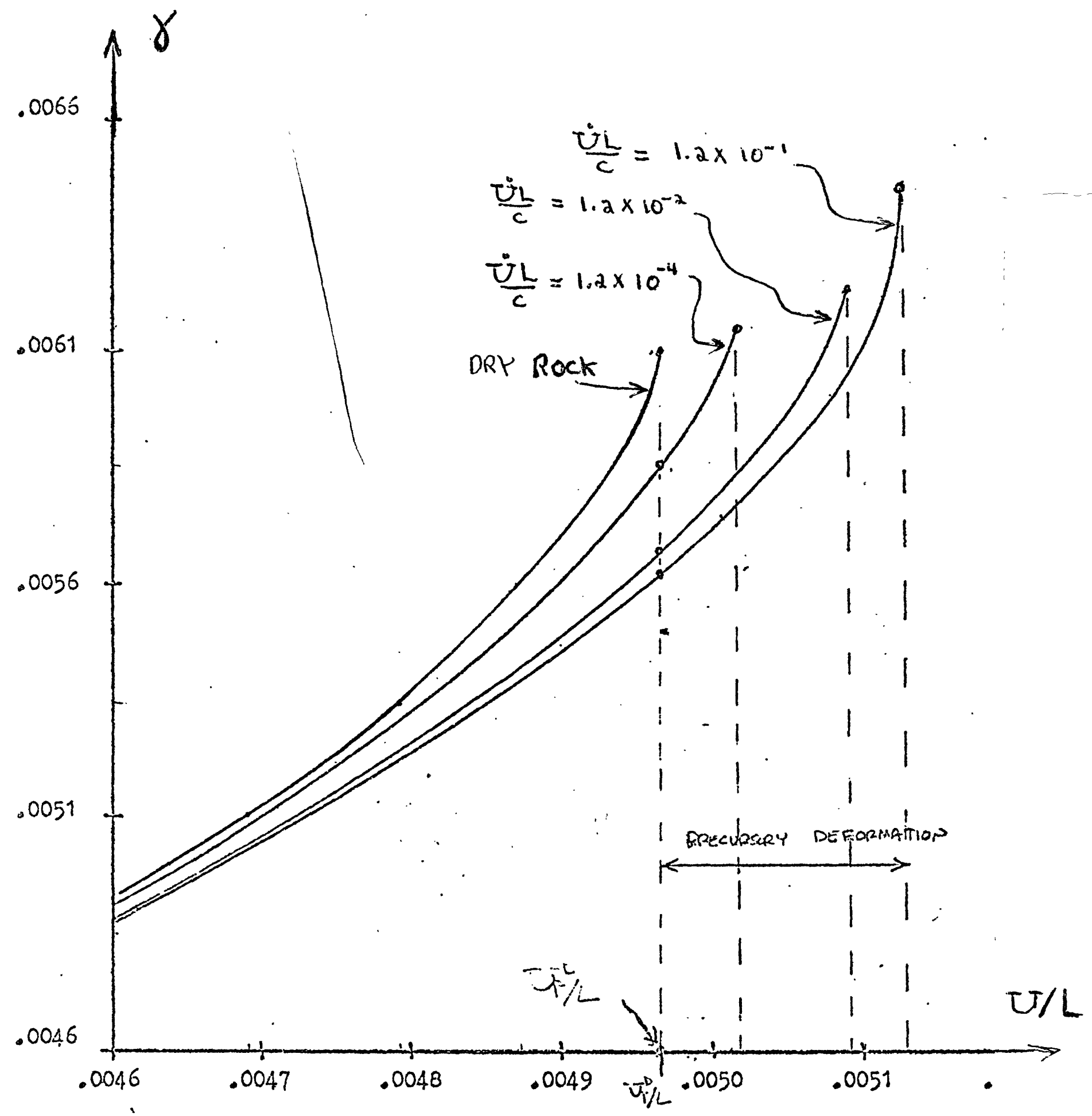

Figure 3 


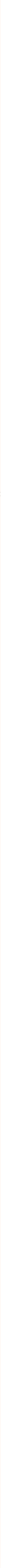




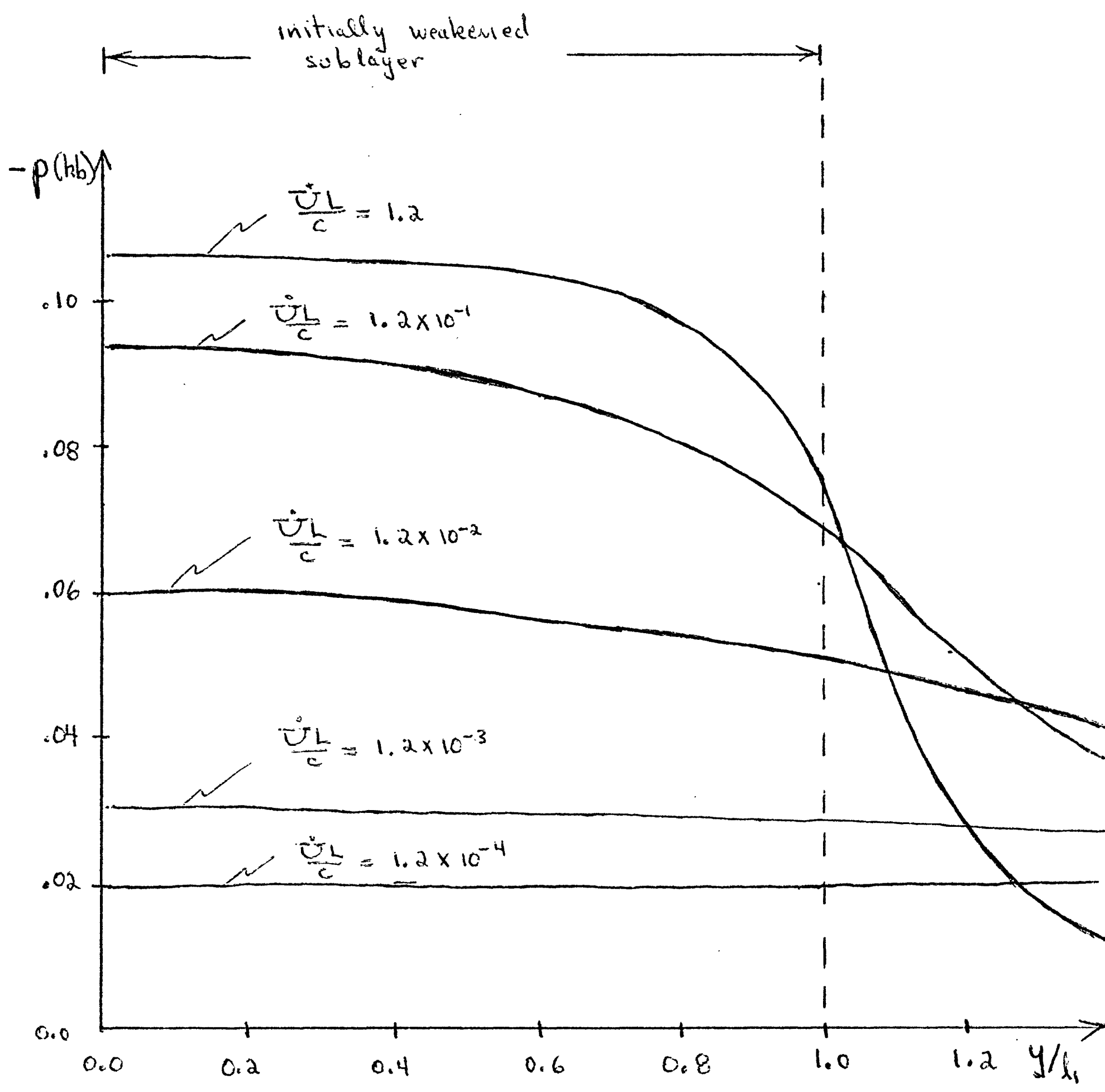

Figure 5 
Appendix I

AN INCLUSION MODEL FOR PROCESSES

PREPARATORY TO EARTHQUAKE FAULTING

John W. Rudnicki

Department of. Theoretical and Applied Mechanics University of Illinois at Urbana-Champaign 61801

June, 1980

To be published in Solid Earth Geophysics and Geotechnology, edited by S. Nemat-Nasser, Applied Mechanics Division Volume, American Society of Mechanical Engineers, New York, 1980. 


\section{ABSTRACT}

Predictions for processes preparatory to earthquakes based on an inclusion model of faulting are reviewed. The inclusion material is assumed to have properties representative of the response of brittle rock in compression; specifically, the inelastic response is strain softening, inhibited by hydrostatic compression and exhibits volume increase (dilation) due to shear. Strain softening of the inclusion materlal leads to a dynamic runaway of inclusion shear strain which is interpreted as the occurrence of an earthquake. For both dry and fluid-saturated rock masses, the model predicts that runaway instability is preceded by a period during which the rate of inclusion straln accelerates relative to the far-field strain rate. However, in a fluid-infiltrated rock mass, the coupling of the deformation with pore fluid diffusion causes a much more pronounced pertod of accelerating inclusion strain and delays the onset of instability beyond its occurrence in a dry rock mass. This transient stabilization arises from two mechanisms: the time dependent elastic response of the fluid-infiltrated material surrounding the inclusion and the dilatant hardening of the inelastic response of the inclusion material. The results for stabilization by dilatant hardening are shown to be consistent with recent laboratory experiments. The analysis is based on generalizations of Eshelby's results for inclusions to nonlinearly deforming inclusions and to a spherical inclusion embedded in a linear fluid-infiltrated elastic solid.

\section{NOMENCLATURE}

a

b

c

$d^{P} \gamma, d_{\varepsilon} P_{\varepsilon} \quad$ inelastic increments of shear and volume strain, respectively

$e_{i j}$

g

G

h

K

$\mathrm{K}_{\mathrm{f}}, \mathrm{k}_{\mathrm{s}}$

$K_{s}^{\prime}$

$K^{\prime}, K_{f}^{\prime}$

major semiaxis of ellipsoldal cavity or inclusion

minor semiaxis of ellipsoidal cavity or inclusion

deviatoric strain

function giving time-dependence of shear strain of spherical cavity wall (see Figure 5)

acceleration of gravity

shear modulus

hardening modulus

drained value of bulk modulus

bulk modulus of pore flufd and solid constituents, respectively

an additional modulus of a porous elastic solid

effective values of bulk moduli due to dilatant hardening

\begin{tabular}{|c|c|}
\hline$k, k, k$ & permeabilities \\
\hline $\mathbf{L}$ & $\begin{array}{l}\text { effective fault length in dislocation model } \\
\text { of ( } 7 \text { ) }\end{array}$ \\
\hline $\mathrm{I}_{1 j \mathrm{k \ell}}$ & tensor of elastic moduli \\
\hline M & elastic modulus, $K /(1+3 K / 4 G)$ \\
\hline m & $\begin{array}{l}\text { fluid mass content per unit volume of por- } \\
\text { ous solid }\end{array}$ \\
\hline$\underline{\mathbf{n}}$ & inward unit normal to cavity boundary \\
\hline $\mathbf{p}$ & pore pressure \\
\hline$q_{1}$ & mass flow rate in $x_{i}$ direction per unit area \\
\hline$Q_{1 j k \ell}$ & $\begin{array}{l}\text { factors relating applied traction to strain } \\
\text { at cavity wall (1) }\end{array}$ \\
\hline Q, R & $\begin{array}{l}\text { nondimensional driving forces proportional } \\
\text { to } i_{\infty}\end{array}$ \\
\hline$s_{\text {Ijkl }}$ & $\begin{array}{l}\text { array of shape factors used in Eshelby's } \\
\text { inclusion solution }\end{array}$ \\
\hline$s_{t j}$ & $\begin{array}{l}\text { deviatoric tensor }\left(S_{k k}=0\right) \\
\text { time }\end{array}$ \\
\hline$t_{D}$ & $\begin{array}{l}\text { diffusion time } a^{2} / c \text { for spherical inclu- } \\
\text { sion }\end{array}$ \\
\hline $\mathbf{v}$ & apparent volume fraction of pore fluid \\
\hline $\mathbf{x}_{1}$ & coordinate \\
\hline$\alpha$ & relaxation constant \\
\hline B & dilatancy factor \\
\hline $\boldsymbol{\gamma}$ & engineering shear strain \\
\hline$\gamma_{p}, \gamma_{0}$ & $\begin{array}{l}\text { strain at peak stress and elastic limit, } \\
\text { see Figure } 2\end{array}$ \\
\hline$\gamma_{B^{\prime}}, \gamma_{D^{\prime}}$ & $\begin{array}{l}\text { values of Inclusion shear strain at runaway } \\
\text { under drained and undrained conditions, } \\
\text { respectively }\end{array}$ \\
\hline$r$ & measure of far-field strain rate \\
\hline$\delta$ & relative slip on frictional fault surface \\
\hline$\delta^{*}$ & $\begin{array}{l}\text { relative slip necessary to reduce shear } \\
\text { stress from } \tau_{p} \text { to } \tau_{r}\end{array}$ \\
\hline$\delta_{1 j}$ & $\begin{array}{l}\text { Kronecker delta, } \delta_{i j}=1 \text { if } i=j, \delta_{i j}=0 \\
\text { if } i \neq j\end{array}$ \\
\hline$\varepsilon_{I j}$ & strain \\
\hline$\varepsilon$ & volume strain, $\varepsilon_{\mathbf{k k}}$ \\
\hline$\mu$ & friction coefficient \\
\hline$\nu_{e}, \nu, \nu_{u}$ & $\begin{array}{l}\text { effective, drained and undrained values of } \\
\text { Poisson's ratios, respectively }\end{array}$ \\
\hline$\lambda$ & $\begin{array}{l}\gamma_{p}-\gamma_{0} \text {, half width of peak in Incluston } \\
\text { stress strain curve }\end{array}$ \\
\hline$\tau$ & shear stress \\
\hline & peak values of shear stress \\
\hline
\end{tabular}


difference between peak and residual values of friction stress

$\rho$

mass density of pore fluld

if stress

$\sigma$ mean normal stress (positive in compression), $\sigma=-\sigma_{\mathbf{k k}} / 3$

$\sigma_{c} \quad$ confining stress in axisymetric compression test

$\bar{\sigma}_{c} \quad$ confining stress minus pore fluid pressure

$\theta$ time non-dimenstonalized by diffusion time

$\xi, \xi_{u} \quad$ shape factors for drained and undrained response

$\zeta \quad 1-K / K_{s}^{\prime}$

Subscripts and superscripts

$\infty$

Inc

denotes value in far fleld

prec

denotes value in inclusion

rev denotes percursor time denotes value in reservoir

\section{INTRODUCTION}

In recent years there has been much attention given to the possibility that earthquakes can be predicted. To determine whether prediction is possible and, if possible, to make predictions on a rational basis, an improved understanding of processes preceding earthquakes is needed. This paper revlews recent results based on an inclusion model of earth faulting which is nechantcally consistent and incorporates material behavior representative of fissured rock. This model predicts that dynamic instability, which is interpreted as the occurrence of an earthquake, is preceded by a period of accelerating strain. Moreover, the coupling of deformation with the diffusion of an infiltrating pore fluid can delay the onset of dynamic instability, set the time scale of the failure process, and cause a more pronounced precursory period of accelerating strain. The time scale and character of this accelerating deformation are such that observable precursors, for example, accelerated strain or tilt of the ground surface, changes in local pore fluld pressure, and in physical properties such as electrical reststivity, radon content of we11s, or selsmic wave speed ratlos, are possible. Some discussion of these results has also been included in recent reviews of the theory of precursory processes in earthquake rupture (1) and the mechanics of earthquake rupture (2).

The inclusion model of earth faulting (3) considers a zone (the inclusion) having mechanical properties which are uniform but different from those of the surrounding material. Specifically, the inclusion is assumed to undergo inelastic deformation representative of brittle rock in compression whereas the surrounding material is regarded as nominally linear elastic. In compression, the stress-strain curve of brittle rock typically exhlbits a peak, 1.e., bxittle rock is strain softening, and inelastic shearing is inhibited by hydrostatic compression and accompanied by inelastic volume increase (dilation). The inclusion may 1dealize either a weakened zone of materlal (3) which, because of past faulting, has a lower threshold to inelastic behavior or a "seismic gap" (1) which is driven into the regime of inelastic behavior because it lags behind the surrounding material in its adjustment to the imposed far field deformation. In elther case, continued loading of the rock mass drives the inclusion material into the strain softening regime. If the slope of the descendIng stress strain curve becomes sufficlently negative, further quasistatic deformation is not possible and a dynamic runaway of inclusion shear strain occurs. This Instability is analogous to that which occurs in a compliant testing machine if the load carrying capacity of the sample decreases more rapidly than the machine can unload.

Rice (1) has pointed out that a general feature of this class of models (and of frictional models based on sliding on a discrete surface in which the friction stress decreases with sliding distance; see, for example, (4, $\underline{5}, 6, \underline{7})$ and the article by Stuart in this volume.) is the increase, prior to instability, of the ratio of inclusion strain increment to far-fleld strain increment. Although this increase may give rise to observable precursors, Rice et a1. (8) and Rice and Rudnickl (9) (hereafter, this reference will be abbreviated as RR) have shown that if the rock mass is fluid-infiltrated, the coupling of deformation to the diffusion of pore fluid can stablilze the rock mass and give rise to a more pronounced period of accelerated deformation. This stabilization is, however, transient and ultimately culminates in Instability, although its onset is delayed beyond the occurrence in the absence of pore fluid. The stabilization by pore fluid effects results from the fact that both the inelastic response of the incluston and the elastic response of the surrounding material are stiffer for rapid load alterations. More precisely, "rapid" neans too rapld to allow time for fluid mass diffusion from material elements and such conditions are said to be undrained. Alternatively, drained conditions occur when load alterations are slow enough that changes in pore fluld pressure are alleviated by fluid mass diffusion. It is well known from analyses of consolidation in soil mechanics (e.g., (10), (11)) that the stiffness of a fluid-infiltrated, but otherwise elastic, material is greater for undrained conditions than for drained conditions. The effects of this timedependent elastic response on faulting have been discussed by Rice and cleary (12), Booker (13), Rice and Simons (14), Rice et al. (8), and RR (9) (Also, see the article by Rice in this volume), and Ruina (15) has discussed the retardation of hydraulic fracture by this effect. The source of the increased stiffness of inelastic response is the tendency of brittle rock to dilate or Increase its pore space when sheared inelastically. For undrained conditions, this dilation tends to cause a decrease in the local pore fluid pressure and, consequent1y, an increase in the effective compressive stress, that is, the total stress minus the pore fluid pressure. Because the Inelastlcity of brittle rock arises from microscale processes which are frictional in nature, this increase in effective compressive stress inhibits further inelastic deformation. This phenomenon is called dilatant hardening and was first discussed for granular media by Reynolds (16). Frank (17) was the first to discuss its relevance of this effect to faulting and Brace and Martin (18) and Martin (19) have observed dilatant hardening in laboratory deformation of rock. Further analyses of dilatant hardening have been given by Rice $(20,1)$, Rudnicki $(21)$, and $R R$.

The analysis of the inclusion model of faulting is based on generalizations of Eshelby's (22, 23) results for ellipsoidal inclusions in linear elastic solids. In the absence of coupling of the deformation with pore fluid diffusion, the generalization is the modest one of recognizing that Eshelby's results can be used to relate the stress and strain in the inclusion to the stress and strain applied in the far-field regardless of whether the inclusion is elastic or inelastic so long as it is homogeneous. For the coupled deformation diffusion problem, the generalization is a more substantial one and to date has been accomplished only for 
spherical inclusions by Rice, Rudnicki and Simons (). Their derivation is based on the solution for a spherical cavity in a fluid infiltrated solid loaded by tractions at the cavity boundary.

of course, the inclusion model of faulting will not be appropriate for all clrcumstances. Because of kinematic constraints or continued faulting in the same region, inelastic deformation associated with faulting may be confined to a narrow zone or fault surface. In this case, a model of faulting as a spreading crack-11ke zone of slip may be more appropriate (e.g., (14), (1), Rice, this volume). Nevertheless, even though faulting is often 1dealized as slip on a distinct surface or intense shear of a narrow zone, this may be the result of localization of inelastic deformation in a much larger volume. Indeed observations of changes of physical properties prior to earthquakes at relatively large distances from the fault suggest such an extent of inelastic deformation.

The review first considers the inclusion model and the application of Eshelby's results to the analysis of runaway instability. Then the effects of fluid-infiltration are reviewed and the recent experimental observations of Martin (19) are discussed in terms of the analysis of $R R$.

\section{INCLUSION MODEL AND RUNAWAY INSTABILITY}

First the case in which the rock mass is dry or deforms under drained conditions is considered. As a1ready mentioned, under drained conditions the deformation occurs so slowly that alterations of pore fluid pressure are alleviated by diffusive mass flux. of course, if the rock mass is fluld-Infiltrated, such conditions will not apply near instability.

The analysis of runaway instablitty is based on recognizing that Eshelby's (22) results, although originally obtained for elastic Inclusions, can be used to relate the stress and strain in an inelastic inclusion to the stress and strain applied in the far field. This result can be obtained by first considering a cavity in an infinite linear elastic solld having moduli $L_{\text {ife. }}$ The solid is loaded at the cavity boundary by unfform tractions $n_{i} \sigma_{i j}$ where $n_{i}$ are the components of the unit normal directed inward from the cavity boundary. Eshelby (22) demonstrated that if the cavity is ellipsoidal, the cavity boundary deforms as if the interior had undergone a uniform strain $\varepsilon_{i j}$ which is given by

$$
\varepsilon_{i j}=Q_{i j k \ell} \sigma_{k \ell}
$$

where the $Q_{i j k \ell}$ depend on the elastic constants and the geometry of the cavity.

Eshelby (22) actually obtained this result by a different route. He first considered an ellipsoidal region ("the incluston") of a linear elastic solid to undergo a change of size and shape which, in the absence of constraint by the surrounding matrix, would be given by the homogeneous stress-free transformation strain $\varepsilon_{i j}^{\mathrm{T}}$. He then showed that the strain which results in the inclusion due to the presence of the constraint by the matrix is also homogeneous and is related to $\varepsilon_{1 j}^{T}$ by

$$
\varepsilon_{i j}=s_{1 j k \ell} \varepsilon_{k \ell}^{T}
$$

where the $S_{\text {ifke }}$ are factors which depend on the shape of the ellipsoid and on ratios of the elastic constants of the matrix. Because the resulting strain of the inclusion is homogeneous, Eshelby (22) was also able to solve problems for inclusions having elastic constants different from those of the matrix and, hence, for cavitles. The $Q_{i j k \ell}$ are related to the $S_{i j k \ell}$ by the following expression (21)

$$
1=\mathrm{L}_{1 j \mathrm{pq}}\left[\delta_{\mathrm{pk}} \delta_{\mathrm{q} \ell}-\mathrm{s}_{\mathrm{pqk} \ell}^{-1}\right] Q_{\mathrm{k} \ell \mathrm{mn}}
$$

where $s_{p q k \ell}^{-1} s_{k \ell 1 j}=\delta_{p l} \delta_{q j}$. General expressions for $s_{1 j k l}$ have been given by Eshelby $(22,23)$ and these have been recorded by Rudnick1 (3). Rudnick1 (3) has also recorded the expressions for the $s_{1 j k \ell}$ in the special case of axisymetric ellipsolds and has tabulated their values for varlous aspect ratios.

The solution for an ellipsoidal inclusion of homogeneous material which is embedded in an infintte 11near elastic body subfected to unfform stress or strain at infinity can be constructed by using (1) and superposition. In particular, the fleld outside the inclusion is identical to that which resuits from the sum of the following: (i) untform stress $\sigma_{i f}^{\infty}$ and the strain associated with $\sigma_{1 j}$ by

$$
\sigma_{i j}^{\infty}=L_{1 j k \ell} \varepsilon_{k \ell}^{\infty}
$$

and (1i) fields which result from loading the cavity boundary by the following tractions

$$
n_{f} \sigma_{f 1}=n_{j}\left(\sigma_{j 1}^{\infty}-\sigma_{j 1}^{\text {lnc }}\right) \text {. }
$$

The displacements of the cavity boundary which result from (11) must be consistent with uniform strain of the interior given by

$$
\varepsilon_{1 j}=\varepsilon_{i j}^{\text {inc }}-\varepsilon_{i j}^{\infty}
$$

Because these strains must be related to the loading (3) by (1), the result is the following "Eshelby relation"

$$
\varepsilon_{i f}^{\text {Inc }}-\varepsilon_{i f}^{\infty}=Q_{i j k \ell}\left(\sigma_{k \ell}^{\infty}-\sigma_{k \ell}^{i n c}\right)
$$

where $\varepsilon_{i j}^{i n c}$ and $\sigma_{i j}^{\text {Inc }}$ can now be identifled as the strain and stress of the inclusion material. Note that (5) has been constructed without reference to the constitutive law of the inclusion material and requires only that the inclusion material be homogeneous, the inclusion shape be ellipsoidal, and the surrounding matrix be Iinearly elastic.

Runaway instability is best illustrated by considering the special case of pure shear and using a graph1cal construction due to Rice $(\underline{1}, \underline{8})$. The component of (5) for pure shear is

$$
\gamma_{\text {inc }}-\gamma_{\infty}=(\xi / G)\left[\tau_{\infty}-\tau_{\text {inc }}\right]
$$

where $\gamma$ is the engineering shear strain, $G$ is the shear modulus of the surrounding material (assumed, for conventence, to be isotropic) and $\xi$ is a shape factor which depends on Poisson's ratio and the geometry of the inclusion.

The value of $\xi$ for a spherical inclusion is (22)

$$
\xi=2(4-5 v) /(7-5 v) \text {, }
$$

where $v$ is Poisson's ratio for the matrix materfal. For flat axtsymmetric and cylindrical inclusions of aspect ratio $a / b \gg 1$, the values of $\xi$ are

$$
\xi=(a / b)[4(1-v) / \pi(2-v)]
$$




$$
\xi=(a / b)(1-v) / \pi
$$

respectively ((3) with correction noted in (1)). For $\nu=0.2, \xi=1.0$ for the spherical inclusion and $\xi=10$ for the axisymetric inclusion with $a / b=18$. Equation (6) is plotted in Figure 1 as the Eshelby Iine of slope $-G / \xi$. Also shown is the (linear elast1c) constitutive law for the surrounding material $\tau_{\infty}=G \gamma_{\infty}$ and a relation for the inclusion shear stress $\tau_{\text {Inc }}$ as a function of the inclusion shear strain $\gamma_{A n}$. The latter is drawn to be representative of the behavfor of brittle rock in compression. For a given value of $\tau_{\infty}$, corresponding to point $A$ in Figure 1 , the corresponding values of $\tau_{\text {fnc }}$ and $\gamma_{\text {inc }}$ are given by the intersection ( $A^{\prime}$ ) of the Eshelby line through point A with the inclusion stress-strain curve. As $\tau_{\infty}$ is Increased, the Eshelby IIne moves outward parallel to itself unt1l it is tangent to the inclusion stress strain curve at $B^{\prime}$. At this point, any further increase in $\tau_{\infty}$ cannot be accommodated by quasistatic deformation and a dynamic runaway of inclusion shear strain occurs. Hence, the condition for runaway instability is the following:

$$
\mathrm{d}_{\text {Inc }} / \mathrm{d} \gamma_{\text {Inc }}=-\mathrm{G} / \xi
$$

Equation (6) can be expressed in differential form as

$d \gamma_{1 n c} / d \gamma_{\infty}=(1+\xi)\left[1+(\xi / G) d \tau_{i n c} / d \gamma_{1 n c}\right]^{-1}$

and, in this form, it is clear that the condition (8) corresponds to the ratio $\mathrm{d} \gamma_{\text {inc }} / \mathrm{d} \gamma_{\infty}$ becoming unbounded. Furthermore, if $\mathrm{d} \tau_{\text {inc }} / \mathrm{d} \gamma_{\text {inc }}$ ingereases continuously, the approach to instability will be marked by a continuous increase in the ratio on the left hand side of (8a).

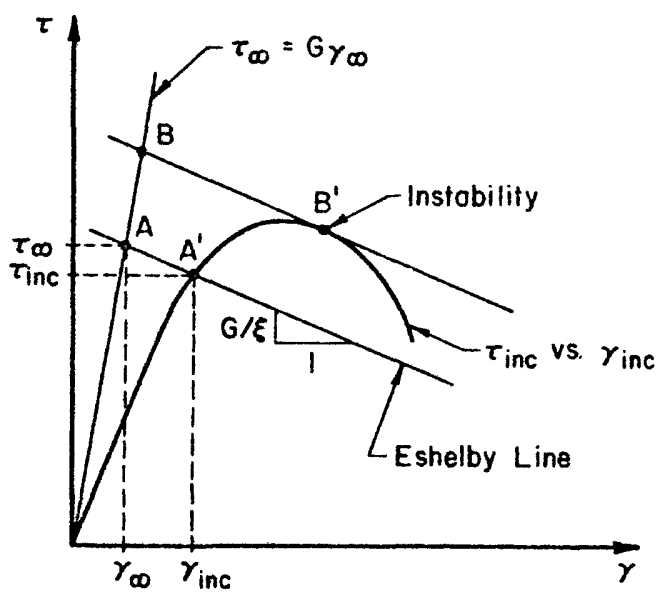

Figure 1. Graphical 1llustration of runaway instabillty. (After Rice (1)).

As a specific example, consider the following relation for $\tau$ versus $\gamma_{\text {inc }}$ which was used by $R R$ in their numerical calculations:

$$
\begin{aligned}
& \tau_{\text {Inc }}={ }^{G} \gamma_{\text {Inc }}, \quad \gamma_{\text {Inc }} \leq \gamma_{0} \\
& \tau_{\text {Inc }}=\tau_{p}-G\left(\gamma_{\text {inc }}-\gamma_{p}\right)^{2} / 2 \lambda, \quad \gamma_{\text {inc }} \geq \gamma_{0}
\end{aligned}
$$

where $\gamma_{0}$ is the strain at the elastic 1fmit, and $\gamma_{p}=\gamma_{0}+\lambda$ is the strain at peak stress $\tau_{p}$ (Figure 2). Using (9) and $\tau_{\infty}=G \gamma_{\infty}$ in (6) ylelds an expression for $\gamma_{\text {inc }}$ as a function of $\gamma_{\infty}$. In Figure 2 the postpeak variation of $\gamma_{\text {inc }}$ (divided by 1 ts value at instability, $\left.\gamma_{\text {inc }}=\gamma_{p}+\lambda / \xi\right)$ is plotted against the following measure of far fleld strain:

$$
r=2 \xi\left[\gamma_{\infty}(1+\xi)-\xi \tau \tau_{p} / G-\gamma_{p}\right] / \lambda
$$

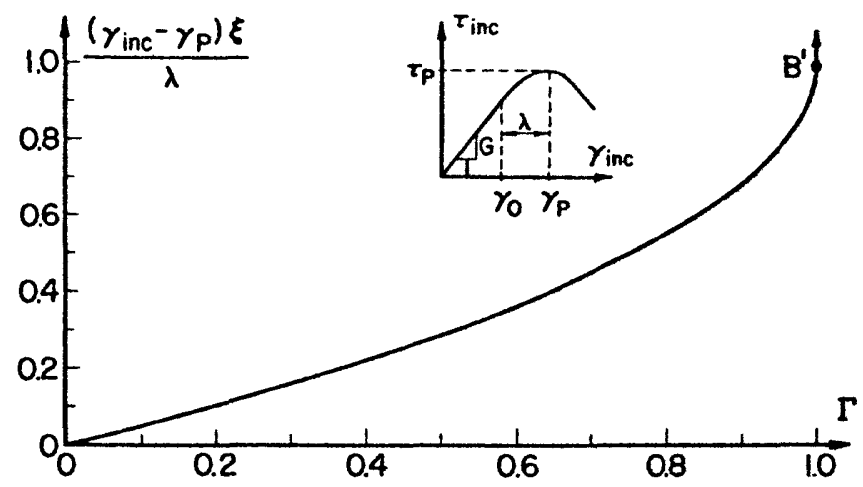

Figure 2. Postpeak variation of inclusion strain as a function of far fleld strain (as measured by r) for inclusion stress strain curve used by $R R$ (see (9)) and shown as inset.

Clearly, the ratio $\mathrm{d} \gamma_{1 n c} / \mathrm{d} \gamma_{\infty}$ increases as instability is approached and as noted by Rice (1), this feature is a general precursory effect for strain softening models. of course, in the earth's crust $\gamma_{\infty}$ changes extremely slowly (In California, measured strain rates are typ1cally less than $1 \mu$ strain per year $(24,25)$ ) and hence the acceleration of inclusion strain may not be detectable until very near instability.

In the model of (3) the inclusion was regarded as weaker than the surrounding material $\left(\tau_{\text {Inc }}<\tau_{\infty}\right.$ ) and Figure 1 has been drawn to be compatible with this interpretation. However, Rice (1) has shown that simllar constructions can be applied to a selsmic gap, that is, an inclusion zone which has lagged behind the surrounding material in adjusting to the far fleld deformation, and to sliding on frictional surfaces for which the friction stress is a function of the amount of relative sliding (also see (7)).

Because brittle rock tends to dilate when sheared inelastically, and because dilation of the inclusion materlal must occur against the constraint of the surrounding matr1x, the response in shear is in general coupled to that for hydrostatic compression. Consequently, the $\tau_{\text {inc }}$ versus $\gamma_{\text {inc }}$ curve which is illustrated schematicaily in Figure 1 should be regarded as that appropriate for in situ behavior. Rudnicki (3), in his treatment of runaway instability for arbitrary ellipsoidal inclusions and general loading conditions, has also given a full analysis of the effects of this coupling between shear and hydrostatic compression. Rudnick1 (3) used a version of the constitutive law proposed by Rudnickf and Rice (26) to model the behavfor of brittle rock and the next section reviews this law for the simple deformation state which is a combination of pure shear and hydrostatic compression. This constitutive law will then be used to illustrate the Inhibiting effects of pressure sensitive and dilatant behavior on runaway instability for a spherical inclusion.

Const1tutive Law for Inclusion Material

The constitutive relation discussed here is a variation of that developed by Rice (20) for an analysis of the stability of dilatant hardening and generallzed by Rudnicki and Rice (26) to multiaxial loading and arbitrary deformation magnitudes. Consider a small element 
of the inclusion material which is subjected to a combination of hydrostatic stress $\sigma$ (In compression) and shear stress $\tau$. If the next increment of deformation tends to cause elastic unloading, the strain increments which are work conjugate to $\sigma$ and $\tau$ are given by

$$
\mathrm{d} \gamma=\mathrm{d} \tau / \mathrm{G}, \quad \mathrm{d} \varepsilon=-\mathrm{d} \sigma / \mathrm{K}
$$

where $G$ and $K$ are the elast1c shear and bulk modu11, respectively. If instead the next increment of deformation tends to cause continued inelastic deformation, the Inelastic increments of strain must be added to those of $(10)$. For constant hydrostatic stress $(d \sigma=0)$, the Inelastic increment of shear strain 18

$$
\mathrm{d}^{\mathrm{P}} \mathrm{\gamma}=\mathrm{d \tau} / \mathrm{h}
$$

where the modulus $h$ may be positive (hardening) or negative (softening) and is related to the slope of the $\tau$ versus $\gamma$ curve by

$$
d \tau / d \gamma=h /(1+h / G) \text { for } d \sigma=0
$$

Because the inelasticity of brittle rock is primarily due to microscale processes which are frictional in nature (e.g., frictional sliding on fissure surfaces and microcracking from the tips of pre-existing fissures), inelasticity is inhibited by an increase in hydrostatic compression. Consequently, for increments In which $d \sigma \neq 0, d \tau$ is replaced in (11) by $d \tau-\mu d \sigma$ where $\mu$ is a coefficient of friction which expresses the elevation of the flow stress due to hydrostatic compression. Uplift in sliding over asperities and tensile cracking from the tips of existing fissures give rise to macroscopic inelastic volume increase (dilatancy) accompanying inelastic shear strain. Hence, the ratio between inelastic increments of volume strain and shear strain is presumed to be fixed by the current stress state:

$$
{ }_{d}^{p} \varepsilon=B d^{p} \gamma
$$

where $\beta$ is the dilatancy factor. In terms of a "yield surface" in $\tau$ versus $\sigma$ space, $\mu$ is the local slope of the yleld surface and $\tan ^{-1}(\beta-\mu)$ is the angle between the inelastic strain increment plotted as a vector $\left(-\mathrm{dP}_{\varepsilon}, \mathrm{dP}_{\gamma}\right)$ and the normal to the yleld surface. Rice (20), Rudnicki and Rice (26), and Rudnicki (3) have estimated values of $\mu$ in the range 0.5 to $1 . \overline{0}$ and $B$ in the range 0.2 to 0.5 from experimental results. In general, all the parameters of the constitutive law may change with deformation but the variation in $h$ is typically most rapid. Cleary and Rudnick1 (27) have given an expression for the tangent modulus in axisymmetric compression in terms of $h, \mu, \beta$ and elastic moduli.

The constitutive law, now written in rate form, for continued inelastic deformation is

$$
\begin{aligned}
& \dot{\gamma}_{\text {inc }}=\dot{\tau}_{\text {inc }} / G+\left(\dot{\tau}_{\text {Inc }}-\mu \dot{\sigma}_{\text {inc }}\right) / h \\
& \dot{\varepsilon}_{\text {Inc }}=-\dot{\sigma}_{\text {Inc }} / K+B\left(\dot{\tau}_{\text {Inc }}-\mu \dot{\sigma}_{\text {Inc }}\right) / h
\end{aligned}
$$

where the designation "Inc" has been included explicit1y. Deformation which tends $t_{0}$ make $\tau_{1 n c}>\mu \dot{\sigma}_{\text {inc }}$ corresponds to continued inelastic deformation whereas that which tends to make $\dot{\tau}_{\text {inc }}<\mu \dot{\sigma}_{1 \text { nc }}$ corresponds to elastic unloading.

Runaway Instability for a Spherical Inclusion

For a spherical inclusion, the Eshelby relations (5) for deviatoric and hydrostatic components decouple.
These equations reduce to the following stmple expresslons

$$
\begin{aligned}
& \dot{\varepsilon}_{\text {Inc }}-\dot{\varepsilon}_{\infty}=(3 / 4 G)\left[\dot{\sigma}_{\text {Inc }}-\dot{\sigma}_{\infty}\right] \\
& \dot{\gamma}_{\text {Inc }}-\dot{\gamma}_{\infty}=(\xi / G)\left[\dot{\tau}_{\infty}-\dot{\tau}_{\text {Inc }}\right]
\end{aligned}
$$

where $\xi=2(4-5 v) /(7-5 v), \sigma=-\sigma_{4 k} / 3, \varepsilon=\varepsilon$ and it has been assumed following Rudnlkki (3) that can be used to relate rates of stress and strain. (15) and (16) to eliminate $\sigma_{\text {inc from (17) yields }}$

$$
\dot{\gamma}_{\text {Inc }}=\dot{i}_{\text {inc }} / G+\dot{\tau}_{\text {inc }} /(h+\mu B M)
$$

where $M=K /(1+3 K / 4 G)$ and, for conventence, it has been as sumed that $\dot{\varepsilon}_{\infty}=0$. This equation makes clear the inhibiting effect that the constraint of the surrounding material has on inelastic deformation of the inclusion: Comparison with (11) reveals that the hardening modulus is elevated from $h$ to an effective value $h+\mu B M$. The condition for runaway instability is easily obtained from (8):

$$
h=-G /(1+\xi)-\mu \beta K /(1+3 K / 4 G) .
$$

It is evident from (19) that for a spherfcal inclusion runaway instability is predicted to occur well after peak stress, that is, when the hardening modulus is less than zero and comparable in magnitude to the elastic shear modulus. Although, as mentioned earlier, runaway is predicted to occur closer to peak stress for narrow inclusions, this calculation and the more general analysis of (3) Indicate the importance of strainsoftening behavior in giving rise to Instability. It has sometimes been suggested, primarily on the basis of a comparison by Walsh $(28)$ of the effective stiffness of a loading apparatus with that of the material surrounding a fault, that such postpeak deformation would not be realized in situ. However, the calculation by Walsh (28) of the effective stiffness of a fault assumed slip on a crack-11ke surface and elastic properties of the fault zone. Although the analysts of (3) agrees with that of (28) in the limiting case of an infinftesimally thin inclusion of nondilatant properties, it is clear that more generally dilatancy, pressure-sensitivity of inelastic behavior, and a finite thickness of the inclusion contribute to the requirement that runaway instabllity occur after peak stress.

Equation (19) assumes that the elastic moduli of the inclusion are equal to those of the surrounding material. However, the microcracking which gives rise to inelastic deformation of brittle rock also causes a degradation of the elastic moduli to 50 to $90 \%$ of their inttial values (3). If the elastic moduli of the inclusion are $G^{*}$ and $K^{*}$, then Rudicki (21) has shown that the value of $h$ at nunaway instabllity is

$$
h=-G^{*} /\left(1+\xi G^{*} / G\right)-\mu S K^{*} /\left(1+3 K^{*} / 4 G\right) \text {. }
$$

For $\mathrm{G}^{*} \ll G$ and $\mathrm{K}^{*} \ll \mathrm{K}$, the expression in the denominator of each term reduces to unity. Rudnick1 (21) also considered the 1 imiting case of an extremely anisotropic spherical inclusion which could deform only by shear and compression or extension relative to a single direction. In this case runaway instability was predicted always to occur after that for the isotropic inclusion.

Rudnick1 (3) also used the analysts of (26) to consider the possibility that deformation in the inclusion would cease to be homogeneous and further deformation would be concentrated in a narrow zone. Rudnicki (3) concluded that conditions for localization of 
deformation would, In general, be met prior to those for runaway instabllity although for very narrow zones both conditions may be satisfied nearly simultaneously. This concluston was based primarlly on the observation that for nearly spherical inclusions the mode of deformation in the inclusion, for example, plane strain versus axisymmetric compression, would be nearly the same as that imposed in the far field whereas for very narrow inclusions the mode of deformation is constrained to be essentially a combination of pure shear and uniaxtal compression. A more detalled analysis of conditions for locallzation of deformation in general ellipsoidal inclusions would be useful. Because conditions for locallzation of deformation are typically met prior to runaway instablilty, the constitutive law of the inclusion material should be regarded as reflecting the overall response of the inclusion rather than that of any speciflc homogeneous material.

\section{EFFECTS OF COUPLED DEFORMATION AND DIFFUSION}

If near surface crustal rock is infiltrated with ground water, predictions of runaway instability based on drained conditions will be altered by coupling between deformation and pore fluid diffusion. More specifically runaway instabllity may be stabilized by the two mechanisms which were mentloned earlfer: time dependent stiffness of the materlal surrounding the inclusion and dilatant hardening. The stabilizing effects of these mechanisms can be 1llustrated in terms of the graphical construction in Figure 1 (1).

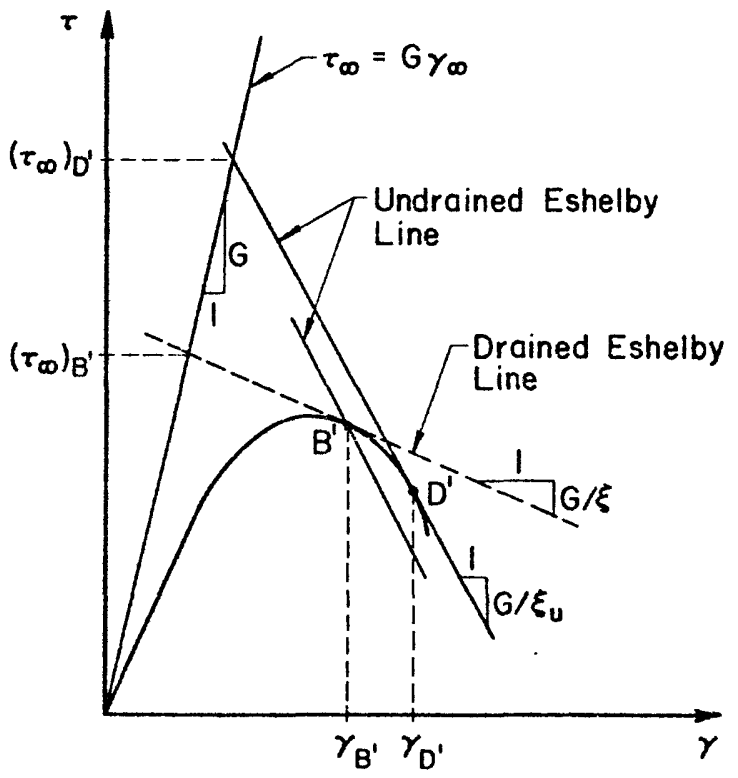

Figure 3. Graphical 1llustration of stabilization by time dependent stiffness of materlal surrounding the inclusion. Instability is delayed from $B^{\prime}$ to $D^{\prime}$. Stiffness changes are greatly exaggerated for illustration.

Figure 3 illustrates stabilization by the t1me dependent stiffness effect. The Eshelby line for drained response is shown as the dashed line of slope $-G / \xi$ which is tangent to the inclusion stress strain curve at 'B'. However, the rapid acceleration of inclusion strain which occurs as $B^{\prime}$ is approached (as 11lustrated in Figure 2) w111 elic1t undrained response. Because the surrounding materlal is elastically stiffer for undrained response, the corresponding Eshelby line is steeper and is not tangent to the inclusion stress strain curve at $\mathrm{B}^{\prime}$. Consequently, instability w1ll not occur at $B^{\prime}$. but instead, is delayed to $D^{\prime}$ where continued softening of the inclusion materfal has caused the stress strain curve to become tangent to the Eshelby Iine for undrained response. During this perlod the inclusion strain increases from $\gamma_{B}$, to $\gamma_{D}$ '.

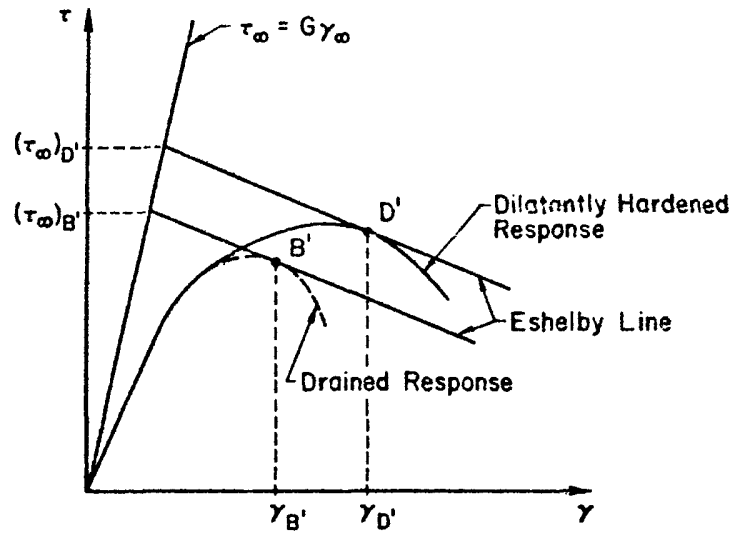

F1gure 4. Graphical 11lustration of stabilization by dilatant hardening of incluston material. Instability is delayed from $B^{\prime}$ to $D^{\prime}$. Magnitude of the dilatant hardening effect is greatly exaggerated for lllustration.

Figure 4 illustrates stabilization by dilatant hardening. Here the slope of the Eshelby line is regarded as constant corresponding to neglecting the time dependent stiffness of the surrounding material. As the inclusion strain approaches $\gamma_{B}$, the instability point predicted by the drained analysis, rapld deformation induces undrained response of the Inclusion matertal. Consequently, the inclusion material does not follow the stress strain curve for drained response (dashed curve) but rather that for undralned response; instability is delayed from $B^{\prime}$ to $D^{\prime}$ where the tangent to the dilatantly hardened response curve has fallen to the slope of the Eshelby 11ne.

Although both Figure 3 and Figure 4 indicate an increase in $\tau_{\infty}$ during the delay from point $B$ ' to point $D^{\prime}$ (The stiffness changes and increases of $\tau_{\infty}$ are greatly exaggerated for 1llustration), the deformation is self-driving once the inclusion straln has exceeded $\gamma_{B}$; that 15 , after this point, the inclusion strain will accelerate to instability at $D^{\prime}$ even if $\tau_{\infty}$ is held constant $(1,21)$. The time which elapses during this period is defined as the precursor time. There is, of course, some arbitrariness in this defini$t$ ion but it is unambiguous in the context of this model and the definition is motivated by the fact that during this perfod the deformation w11l be much more rapid than the tectonic strain rate. Consequently, the detection of precursory phenomena may be possible during this period.

To predict the precursor time and to analyze quantitatively the increase of inclusion strain toward instability, it is necessary to obtain Eshelby relations analogous to (5) for a linear porous elastic fluidinfiltrated ("Biot") solld. The governing equations for such a solid were first established by Biot (11) and these are reviewed in the next section. The Eshelby relations can, in principle, be obtained by the same steps outlined in the derivation of (5): First, the problem of an ellipsoldal cavity loaded at its boundary is solved; if the resulting (now time-dependent) deformation of the cavity wall is compatible with homogeneors strain of the interlor, then the solution for the inclusion can be obtained by superposition. Thus far, however, only the solution for a spherical cavity has been obtalned (8). This solution, its use in obtaining 
Eshelby relations, and the application of these relations to the analysis of transient stabilization of runaway instability (9) will be reviewed in the following subsections.

Governing Equations for a Biot Solid

Although the governing equations for a linear porous elastic fluid-infiltrated solid were first derived by Biot (11), Rice and Cleary (12) have given a more elucidating rearrangement of these equations. Rice and Cleary (12) demonstrated that in the limits of drained and undrained response, the total stress $\sigma_{i j}$ is related to the strain of the solid matrix $\varepsilon_{i j}$ by the usual relation of linear elasticity; that is

$$
\sigma_{i j}=2 G\left[\varepsilon_{i j}+v_{e} \varepsilon_{k k} \delta_{i j} /\left(1-2 v_{e}\right)\right]
$$

where $\delta_{1 f}$ is the Kronecker delta, $G$ is the shear modulus, and $v$ is an effective value of Poisson's ratio. This Polsson's ratio has the value $v$ for drained response and $v_{u}$ for undrained response where $v \leq v_{u} \leq 1 / 2$. The upper 1 imit is attained for separately incompressible constituents and the lower for highly compressible pore fluid. Thus, the source of the elastically stiffer response for undrained conditions, and hence the stabilizing effect, is an increase in the effective value of Poisson's ratio.

More generally, the stress $\sigma_{i j}$ and the change in fluid mass content per unit volume $m$ are related linearly to the strain $\varepsilon_{i j}$ and the alteration in pore fluid pressure $p$. The pote fluid pressure is defined precisely as the value of the pressure in a reservoir of homogeneous pore fluid which, when connected to a small element of porous material, would equilibrate any fluid mass flux from the element. Such an element, although it may contain several interconnected pores or fissures, would be regarded in this continuum formulation as a "point". Very rapid load alterations, for example, on the time scale of wave propagation, would cause the pressures in these pores or fissures, to be different and, under these circumstances, the characterization of the pore fluid by a single pressure would be inadequate. Cleary (29) and $0^{\prime}$ Connell and Budiansky (30) have discussed the response in this dynamic regime. Hence, the undrained response which is regarded as instantaneous in the Biot formulation assumes there is sufficient time for pressure equilibration among cavities occupying a single "point".

The complete set of constitutive equations is the following:

$$
\begin{aligned}
& \sigma_{i j}=2 G\left[\varepsilon_{i j}+v \varepsilon_{k k} \delta_{i j} /(1-2 v)\right]-\zeta p \delta_{i j} \\
& m-m_{0}=\zeta \rho\left[\varepsilon_{k k}+\zeta p(1-2 v)\left(1-2 v_{u}\right) / 2 G\left(v_{u}-v\right)\right] \\
& q_{i}=-\rho \kappa \partial p / \partial x_{i}
\end{aligned}
$$

where $m_{0}$ is the value of $m$ in the unloaded state, $p$ is the mass density of the pore fluid, and (23) is Darcy's law which relates $q_{1}$, the mass flow rate in the $x_{1}$ direction per unit area, to the gradient of pore fluid pressure; $K$ is a permeability which is of ten expressed as $k=k / \mu$ where $\mu$ is the fluid viscosity and $k$ has the dimensions of length squared (usually measured in millidarcies; $1 \mathrm{md}=10^{-11} \mathrm{~cm}^{2}$ ) or as $k=\bar{k} / \rho_{0} g$ where $g$ is the acceleration due to gravity and $k^{\circ}$ has dimensions of velocity; $\zeta=1-K / K_{s}$ ' where $K(=2 G(1+v) / 3(1-2 v))$ is the bulk modulus of the porous matrix material and $K_{s}^{\prime}$ is an empirical constant which, under conditions stated by Rice and Cleary (12) (also see (31)), is identical to the bulk modulus of the solid constituents.
For drained conditions, any alterations in pore fluid pressure are dissipated, $p=0$, and (21) reduces to (20) with $\nu_{e}=v$. For undrained conditicns, there is insufficient time for fluid mass diffusion from material elements, $m=m_{0}$, and the change in pore fluid pressure is obtained by equating the right hand side of (22) to zero. Substituting the resulting value for $p$ into (21) again reduces this equation to (20) with $v_{e}=v_{u}$.

These constitutive equations must be combined with the strain displacement relation

$$
\varepsilon_{i j}=\left(\partial u_{i} / \partial x_{j}+\partial u_{j} / \partial x_{i}\right) / 2
$$

and field equations expressing equilibrium

$$
\partial \sigma_{i j} / \partial x_{i}=0
$$

and conservation of fluid mass

$$
\underline{\nabla} \cdot \underline{q}+\partial \mathrm{m} / \partial \mathrm{t}=0
$$

Substituting Darcy's law (23) in this last equation (26) and rearranging by using (25), (21), and (22) yields a homogeneous diffusion equation for the fluid mass content $m(\underline{12})$

$$
\nabla^{2} \mathrm{~m}=\frac{1}{\mathrm{c}} \frac{\partial \mathrm{m}}{\partial \mathrm{t}}
$$

where $\nabla^{2}(\ldots)=\partial^{2}(\ldots) / \partial x_{k} \partial x_{k}$. The diffusivity $c$ can be expressed as (12)

$$
c=\left[2 G \frac{(1-v)}{(1-2 v)}\right]\left[\frac{\kappa\left(v_{u}-v\right)}{\zeta^{2}(1-2 v)\left(1-v_{u}\right)}\right]
$$

where the first term in square brackets is the modulus governing one dimensional straining and is usually called the consolidation coefficient in soil mechanics. Note that the diffusivity $c$ rather than the permeability $K$ is the material parameter which sets the time scale of diffusion. Although these quantities are proportional, the diffusivities corresponding to the same value of permeability may be quite different. For example, Rice and Simons (14) give a diffusivity of $10^{-2} \mathrm{~cm}^{2} / \mathrm{sec}$ as representative of the elastic behavior of clay corresponding to a permeability $\bar{k}=10^{-7}$ $\mathrm{cm} / \mathrm{s}$ (or $\mathrm{k}=0.1 \mathrm{md}$ ); however, for a Ruhr sandstone with a permeability twice that of the clay, Rice and Cleary (12) give a diffusivity of $53 \mathrm{~cm}^{2} / \mathrm{s}$ which is more than three orders of magnitude larger than that of the clay.

Values of $c$ determined from laboratory measurements (see (12) for a summary) are typically at least one to two orders of magnitude smaller than values estimated from field observations. Obviously, large foints and fissures which can contribute substantially to fluid transport in the field are not present in laboratory size samples. Based on a variety of fluid observations, Anderson and Whitcomb (32) suggested a fleld diffusivity of $1 \mathrm{~m}^{2} / \mathrm{s}$ and Rice (1) has estimated $\mathrm{c}=0.1 \mathrm{~m} / \mathrm{s}$ from measurements by Kovach et al. (33) of water level changes in wells. A recent literature survey by Li (see (34)) has indicated that these values are consistent with published evidence on fleld diffusivities near fault zones.

Rice and Cleary (12) have tabulated values of $v$ and $v_{u}$ ('as well as other parameters) inferred from laboratory tests on rock. However, the presence of large foints and fissures also affects the values of $\nu$ tending to reduce $v$ and increase $v_{u}(\underline{9}, \underline{12}, \underline{30}, \underline{35})$. 
Unfortunately, there appears to be no direct source of field values for these parameters. Neither corresponds to values inferred from selsmic wave speed ratios. Rice and Rudnicki $(\underline{9})$ have given some estimates by using the self-consistent calculations of $O^{\prime}$ Connell and Budlansky (35) and a value of crack density parameter suggested by them on the basis of a comparison of their calculations with observations of wave speed ratlos prior to the San Fernando earthquake.

\section{Eshelby Relations for a Fluid-Infiltrated Solld}

The Eshelby relations for a spherical inclusion in a fluld-infiltrated solid can be constructed by first solving the equations of the last section for sudden application of traction and pore fluld pressure at the wall of a spherical cavity. The solution for sudden application of pore pressure $p_{0}$ and purely radial total stress $\sigma_{0}$ has been given by Rice and cleary (12). As discussed in (8), the displacement of the cavity wall is independent of $p_{0}$ and is compatible with a uniform volume strain of the cavity interlor. This strain is related to the applied stress by

$$
\varepsilon=(3 / 4 G) \sigma_{0}
$$

Comparison with (16) reveals that this agrees exactly with the result for a linear elastic solid. The fluld mass flow out of the cavity can be calculated from the pressure distribution outside the cavity and Darcy's law:

$q(r=a, t)=-\rho \kappa \partial p / \partial r=\rho k\left[1+a /(\pi c t)^{1 / 2}\right] p_{0}$

where $q$ is the radial mass flux and $a$ is the radius of the cavity. RIce et al. (8) show that fluid mass conservation on the surface of the cavity leads to the following expression:

$\dot{m}(t)=-\left(3 p k / a^{2}\right) p_{0}\left[1+a /(\pi c t)^{1 / 2}\right]$

where $\dot{m}(t)$ is now interpreted as the rate at which fluid mass of the cavity increases.

The boundary conditions for sudden application of shear traction to the cavity wall are given by Rice et a1. (8) as

$$
\left.\begin{array}{rl}
x_{i} \sigma_{i j} & =-x_{1} s_{1 j} \\
p & =0
\end{array}\right\} r=\left(x_{1} x_{1}\right)^{1 / 2}=a, t>0 .
$$

where $S_{1 j}$ is an arbitrary deviatoric tensor, 1.e., $\operatorname{tr} \underset{\sim}{S}=s_{k k}^{1 j}=0$, and $x_{1} / a$ is the component of the outward unit normal to the cavity surface. Rice et al. (8) constructed the solution by recognizing that materfal isotropy, spherical symmetry and linearity require the displacement to have the form

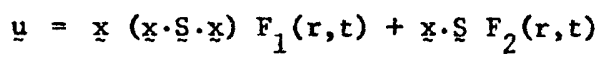

where $\underset{2}{x} \cdot \underline{S} \cdot \underline{x}=x_{1} S_{i f} x_{j}, r=\left(x_{1} x_{1}\right)^{1 / 2}$ and $F_{1}(r, t)$ $F_{2}(r, t)$ are functions to be determined. For the same reasons, the alteration in fluid mass content must have the form

$$
m-m_{0}=(\underset{\sim}{x} \cdot \underline{s} \cdot \underset{\sim}{x}) g(x, t)
$$

unknown functions $F_{1}, F_{2}$, and $g$ can be determined by solving the equations which result from substituting (33) and (34) into the governing equations.

Rice et al. (8) demonstrate that the cavity boundary deforms as if the interior had undergone a uniforn deviatoric strain given by

$2 G e_{1 j}=\left[\xi_{u}+f\left(c t / a^{2}\right)\left(\xi-\xi_{u}\right)\right] s_{1 j}$ at $r=a$

where $\xi=2(4-5 v) /(7-5 v), \xi_{u}=2\left(4-5 v_{u}\right) /\left(7-5 v_{u}\right)$ and the function $f\left(c t / a^{2}\right)$ has the liniting values $f(0)=0$ and $f(\infty)=1$. Hence, the longtime and instantaneous responses are given by the classical elasticity solution with Polsson's ratio equal to its drained and undralned value, respectively. The form of (35) is Independent of the boundary condition on the fluld mass at the cavity wall although the spectfic time-dependence of $f\left(c t / a^{2}\right)$ does depend on this boundary condition. The equation governing the time dependence of the cavity strain is obtained by substituting (34) into the diffusion equation (27)

$$
\frac{\partial^{2} g}{\partial r^{2}}-\frac{4}{r} \frac{\partial g}{\partial r}=\frac{1}{c} \frac{\partial g}{\partial t}
$$

and this must be solved subject to the inftial condition $g(x, t=0)=0$ (because undrained conditions apply at $t=0$ ) and to the boundary condition derived from the second of equations (32). The function $f\left(c t / a^{2}\right)$ is related to $g(a, t)$ through the boundary condition on the traction. For $p=0$ at the cavity boundary, the function $f\left(c t / a^{2}\right)$ is shown in Figure 5 (As shown in (8), $f\left(c t / a^{2}\right)$ exhibits on1y a slight dependence on material properties over the range of interest). Note that $f\left(c t / a^{2}\right)$ reaches more than $50 \%$ of its drained value for $t=0.1 \mathrm{a}^{2} / \mathrm{c}$ so that this time rather than $a^{2} / c$ might be regarded as characteristic.

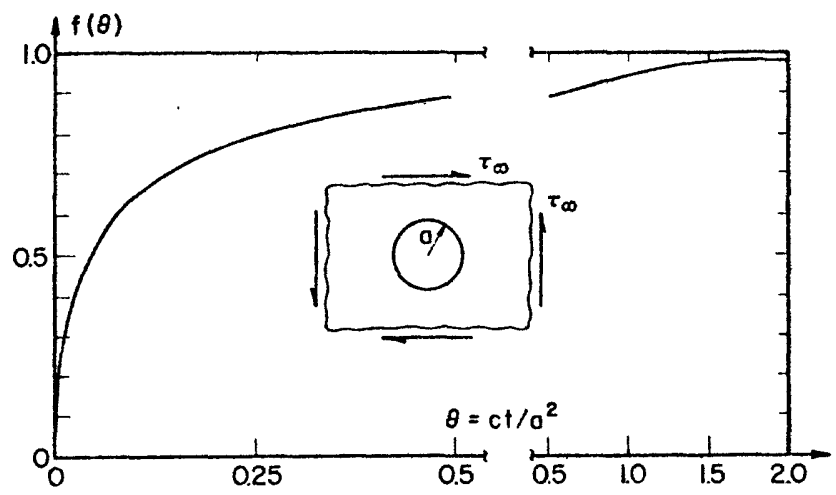

Figure 5. Time dependent shear strain of the cavity wall $(r=a)$ due to sudden application of $\tau$. $f\left(c t / a^{2}\right)=[\gamma(a, t)-\gamma(a, 0)] /[\gamma(a, \infty)-\gamma(a, 0)]$ From ().

The solutions (29), (31), and (35) can be generalIzed by straightforward superposition to arbitrary time varlation of the loads at the cavity boundary. The Eshelby relations for the spherical inclusion are then obtained by combining these solutions with those due to uniform stress and strain in the same manner as was done in deriving (5). The resulting Eshelby relations are

$G\left[\varepsilon_{\text {inc }}(t)-\varepsilon_{\infty}(t)\right]=(3 / 4)\left[\sigma_{\text {inc }}(t)-\sigma_{\infty}(t)\right]$ 


$$
\begin{aligned}
& \dot{\mathrm{m}}_{\text {Inc }}(t)=-\left(3 \rho \mathrm{k} / \mathrm{a}^{2}\right)\left(\mathrm{p}_{\text {Inc }}(t)-\mathrm{p}_{\infty}(t)\right. \\
& \left.+\int_{-\infty}^{t} \frac{a}{\left[\pi c\left(t-t^{\prime}\right)\right]^{1 / 2}}\left[\dot{p}_{\text {inc }}\left(t^{\prime}\right)-\dot{p}_{\infty}\left(t^{\prime}\right)\right] d t^{\prime}\right\} \\
& G\left[\gamma_{\text {inc }}(t)-\gamma_{\infty}(t)\right]=\int_{-\infty}^{t}\left\{\xi_{u}+\left(\xi-\xi_{u}\right) f\left[c\left(t-t^{\prime}\right) / a^{2}\right]\right\} \\
& \text { - }\left[\dot{\tau}_{\infty}\left(t^{\prime}\right)-\dot{\tau}_{\text {Inc }}\left(t^{\prime}\right)\right] d t^{\prime}
\end{aligned}
$$

where the last equation, which was derived from (35), has been speclalized to a single component of shear. In equation (38) for $\dot{\mathrm{m}}_{\text {inc }}(t)$, it has been assumed that $p_{\infty}$ and $\sigma_{\infty}$ are such that $m_{\infty}$ is constant. It has also been assumed that the pore fluid pressure in the inclusion is homogeneous. This cannot, of course, be true exactly but it is likely to be a good approximation if the inclusion is more heavily fissured than the surrounding material as is plausible for the present application.

Equations (37), (38) and (39) relate the state in the inclusion to the state in the far fleld in a manner which is analogous to (5). If constitutive relations for $\tau_{\text {inc }}$, $\sigma_{\text {inc }}$ and $m_{\text {inc }}$ are given in terms of $\gamma_{\text {Inc }}, \varepsilon_{\text {Inc }}$, and $p_{\text {Inc }}$, these equations (with the 1inear elastic relations for the surrounding material) are sufficlent to determine the time-dependent response of the Inclusion in terms of the loads applied in the far field. RR (9) have carried out this procedure to analyze in detai1 the transient stabilization of runaway instability by coupled deformation diffusion effects. In their analysis, $R R$ (9) consider separately stabilization by the time-dependent stiffness of the surrounding material and by dilatant hardening of the inclusion. Their results are reviewed in the succeeding subsections.

\section{Stabilization by Time-Dependent Stiffness of}

\section{Surroundings}

As shown schematically in Figure 3 and analytically by (35), the unloading stiffness of the material surrounding the inclusion is $G / \xi$ for drained conditions and $G / \xi_{u}$ for undrained conditions where $\xi$

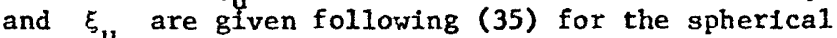
inclusion. Hence, the ratio of drained to undrained response is $\xi / \xi_{\text {and }}$ ance $\xi<\xi$, Instablitty can be delayed as shown in Figure 3. Based on the earlier mentioned estimates of in situ values for $v$ and $v_{u}, R R$ suggest that representative values of $\xi / \xi_{\text {lie }}$ in the range from 1.10 to 1.25 .

If dilatant hardening of the inclusion is neglected, the stabilization by time-dependent stiffness can be addressed with reference to equation (39) without regard to (37) and (38). Specifying a constitutive relation for $\tau_{\text {inc }}$ versus $\gamma_{\text {inc }}$ in (39) yields a nonlinear integral equation for the time-dependent strain in the inclusion. $\mathrm{RR}$ use $(9)$ for $\tau_{\text {inc }}$ versus $\gamma_{\text {inc }}$ and express the result for $\bar{\gamma}_{\text {inc }}$ in nondimensional form as

$$
\frac{\gamma_{\text {Inc }}-\gamma_{p}}{\gamma_{D^{\prime}}-\gamma_{p}}=r\left(\theta ; R, \xi / \xi_{u^{\prime}}, \xi\right)
$$

where $\gamma_{D}$ is the straln at which instability occurs, $\theta=c t / a^{2}$ is time nondimensionalized by the diffusion time and

$$
R=\xi(1+\xi)\left(a^{2} / c\right)\left(\dot{\tau}_{\infty} / G \lambda\right)
$$

Is a nondimensional forcing term which is proportional to the product of the diffusion time $a^{2} / c$ and the far field stress rate $\dot{\tau}_{\infty}$ : Because the tectonic stress rate represented by $\dot{\tau}_{\infty}$ is very small (measurements of strain accumulation in Southern California suggest $\dot{\tau}_{\infty} \approx 10 \mathrm{kPa} /$ year $(0.1 \mathrm{bar} /$ year $\left.)(24,25)\right)$, $\mathrm{R}$ is typ $1-$ cally a small number and $R R$ investigated values ranging from $10^{-4}$ to 3 . The solution was found to be relatively insensitive to the value of $\xi$. A representative result is shown schematically in Figure 6 where the dashed curve shows the response in the absence of pore fluid effects. The nondimensional precursor time $\theta_{\text {prec }}=c t_{\text {prec }} / a^{2}$ is defined as the time which elapses between the point $B^{\prime}$ at which runaway would have occurred in the absence of pore fluid effects and final instability, at $D^{\prime}$. RR give a plot of $\theta_{\text {pec }}$ versus $R$ for $\xi / \xi_{u}=1.10$ and $\xi / \xi_{u}=1.25$. Although the dimensional precursor time $t_{\text {prec }}$ does increase with the size of the inclusion zone, it is not proportional to the diffusion time $a^{2} / c$ : the precursor time increases much more slowly with inclusion size than does the diffusion time. RR give extensive numerical results for representative ranges of parameters. The parameters describing the inclusion stress strain curve are taken to be the following: $\tau_{\mathrm{p}}=100 \mathrm{MPa}(=1 \mathrm{kbar})$, $\lambda=2.5 \times 10^{-3}, \gamma_{p}=6.25 \times 10^{-3}$ and $G=20 \mathrm{GPa}$ $(=200 \mathrm{kbar})$. For inclusion radil of 1 to $5 \mathrm{~km}$, $\dot{\tau}_{\infty}=0.1 \mathrm{MPa} /$ year ( $1 \mathrm{bar} /$ year $), c=0.1 \mathrm{~m}^{2} / \mathrm{sec}$ and $1.0 \mathrm{~m} / \mathrm{sec}$, and $\xi / \xi_{\mathrm{u}}=1.10$ and 1.25 , they find precursor times of 15-400 days based on stabllization by time-dependent stiffness of the surroundings, although they suggest that the lower limit may be more indicative of when precursory vartations could easily be detected.

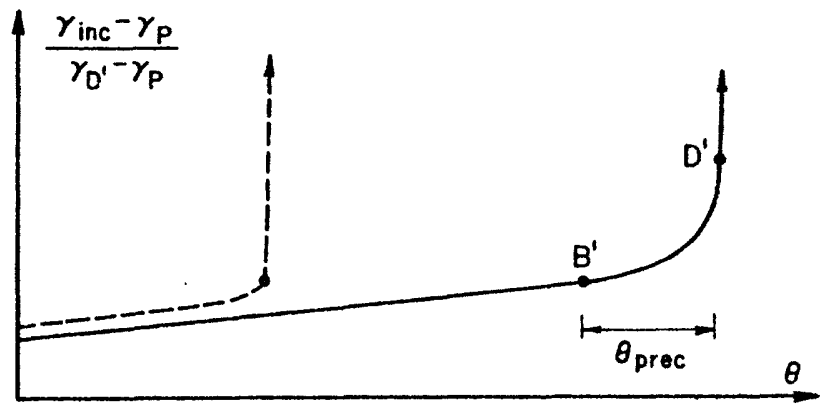

Figure 6. Typical postpeak variation of inclusion strain with $\theta=c t / a^{2}$ as calculated in (9) for stabilization by the time dependent stiffness effect. Far field loading rate is constant. Dashed curve shows drained response (as in Figure 2). Points $B^{\prime}$ and $D^{\prime}$ correspond to points in Figure 3. For numertcal values, see (9).

Rice (1) has also calculated precursor times for the time-dependent stiffness mechanism by approximating the response function $f(\theta)$ in (39) by

$$
f(\theta) \simeq 1-e^{-\alpha \theta}
$$

where $\alpha$ is chosen to give the best fit. This corresponds to approximating the actual response of a fluidInfiltrated solid by that of a standard linear solid with long and short time responses chosen to colncide with drained and undrained behavior, respectively. Although the approximation to $f(\theta)$ is not a particularly good one, the precursor times calculated by Rice (1) agree well with those calculated by $R R$. Because this 
approximation does result in a great saving of computation time, it may be useful in future calculations.

\section{Stab1lization by D1latant Hardening}

For the analysis of dilatant hardening, it is necessary to generalize the constitutive relations (equations (14) and (15)) of the Inclusion to Include the effects of variations in pore fluid pressure. Th1s can be accomplished by using the effective stress princlple: the hydrostatic stress $\sigma$ is to be replaced in (14) and (15) by a linear combination of $\sigma$ and $p$. For elastic deformation Nur and Byerlee (31) (also see (12)) have shown that the correct form of the effective stress is

$$
\sigma-\left(1-\mathrm{K} / \mathrm{K}_{\mathrm{B}}\right) \mathrm{p}
$$

where, as before, $\mathrm{K}$ is the bulk modulus of the matrix material and $K_{s}$ is the bulk modulus of the solid constituents. This form of the effective stress assumes isotroplc behavior, but Carroll (36) has given the generalization for anisotropy. Rice (37) has shown that for inelastic deformation typlcal of brittle rock, that is, inelastic deformation arising from frictional sliding at sharp asperity contacts and microcracking from sharp-tipped fissures, the appropriate form of the effective stress is $\sigma-p$. Making these replacements In (14) and (15) ylelds

$$
\begin{aligned}
& \dot{\gamma}=\dot{\tau} / G+[\dot{\tau}-\mu(\dot{\sigma}-\dot{\mathrm{p}})] / \mathrm{h} \\
& \dot{\varepsilon}=-\left[\dot{\sigma}-\left(1-\mathrm{K} / \mathrm{K}_{\mathrm{s}}\right) \dot{\mathrm{p}}\right] / \mathrm{K}+\beta[\dot{\tau}-\mu(\dot{\sigma}-\dot{\mathrm{p}})] / \mathrm{h}
\end{aligned}
$$

where the subscript "Inc" has been omitted. One add1tional constitutive equation is needed for $m$ the change in fluld mass content per unlt volume. The fluid mass content is related to the apparent volume fraction $v$ by $m=\rho v$. Linearizing this relation ylelds

$$
\mathrm{dm}=\mathrm{v}_{\mathrm{o}} \mathrm{d} \rho+\rho_{0} \mathrm{dv}
$$

where the subscript " 0 " denoting the reference value will be implicit in the subsequent equations. The change in density is given

$$
\mathrm{d} \rho=\rho \frac{\mathrm{d} p}{\mathrm{~K}_{\mathrm{f}}}
$$

where $K_{f}$ is the bulk modulus of the pore fluid. The increment of apparent volume fraction can be written as the sum of elastic and Inelastic portions: The elastic portion can be expressed in terms of stresses by using reciprocity relations $(38,20)$ and $R$ ice $(20,37)$ has shown that $\mathrm{dP}_{\mathrm{V}}=\mathrm{dP}_{\varepsilon}$ under the same circumstances for which $\sigma-p$ is the appropriate form of the effective stress. The final result is

$\frac{\dot{m}}{\rho}=\frac{\mathrm{v} \dot{\mathrm{p}}}{\mathrm{K}_{\mathrm{f}}}-\left[\frac{1}{\mathrm{~K}}-\frac{1}{\mathrm{~K}_{\mathrm{s}}}\right](\dot{\sigma}-\dot{\mathrm{p}})-\frac{\mathrm{v}}{\mathrm{K}_{\mathrm{s}}} \dot{\mathrm{p}}+\beta[\dot{\mathrm{\tau}}-\mu(\dot{\sigma}-\dot{\mathrm{p}})] / \mathrm{h}$

The stabilizing effect of dilatant hardening can be illustrated simply from (41), (42) and (45) before considering the more complicated results of the inclusion model. For drained conditions, $p=0$ and $d \tau / d \gamma$ is given by (12) (with $\dot{\sigma}=0$, for convenience). If, however, the response is undrained, $\dot{m}=0$ and the resulting value of $p$ can be calculated from (45):

$$
\dot{\mathrm{p}}=-\beta \mathrm{K}^{\prime} \dot{\mathrm{\tau}} /\left(\mathrm{h}+\mu \beta \mathrm{K}^{\prime}\right)
$$

where again for conventence, $\dot{\sigma}=0$ and the modulus $K^{\prime}$
Is defined by the following expression:

$$
\frac{1}{K^{\prime}}=\frac{1}{K}+\frac{v}{K_{f}}-\frac{1+v}{K_{s}}
$$

Substituting (46) 1nto (41) with $\dot{\sigma}=0$ reveals that $\mathrm{d} \tau / \mathrm{d} \gamma$ is given by an expression analogous to (12) but with the hardening modulus $h$ replaced by the augmentec value $h+\mu B K^{\prime}$, that $i_{s}$,

$$
(\mathrm{d} \tau / \mathrm{d} \gamma)_{\text {undrained }}=\frac{h+\mu \beta K^{\prime}}{1+\left(h+\mu \beta K^{\prime}\right) / G}
$$

For representative values of $\mu, \beta$ and $K^{\prime}$ this increase in $h$ can be significant. For heavily fissured rock in which most of the porosity is due to long narrow cracks and a pore flutd bulk modulus comparable to that of Iiquid water $\left(K_{f}=2.2 \mathrm{GPa}(=22 \mathrm{kbar})\right), \mathrm{K}^{\prime} \simeq \mathrm{K}$. If, however, $K_{f}$ is reduced by h1gh temperatures, low pore pressure or entrapped gases $K^{\prime} \simeq K_{f} / v$. of course for the incluston model, the above result is modified by the effects due to the constraint of the surrounding material. In particular, $R R$ show that for the 1ncluston model the undrained response is given by (48) with $\mathrm{K}^{\prime}$ replaced by

$$
K_{f}^{\prime}=M\left[1+(K / M)\left[\left(v K / K_{f}\right)\left(M_{s} / M\right)\left(1-K_{f} / K_{s}\right)+\left(1-K / K_{s}\right)\right]^{-1}\right\}
$$

where $M=K /(1+3 K / 4 G), \quad M_{s}=K_{s} /\left(1+3 K_{s} / 4 G\right)$ and $K_{f}^{\prime}$ exhibits the same limiting behavior as $K^{\prime}$.

$R R$ give a full analysis of dilatant hardening fol the inclusion and the detalls will not be discussed her Because they neglect the time-dependent stiffness effects which were discussed earlier, they use (6) rather than (39) as the Eshelby relation for shear. Equation (6) is used with the other Eshelby relations (37) and (38) and with the constitutive relations (41), (42), and (45) to obtain a set of two coupled equations, an ord1nary differential equation and an integral-differential equation, which describe the time dependent response of the inclusion material. The $\tau_{\text {inc }}$ versus $\gamma_{\text {inc }}$ curve is again chosen to be that given by (9) and because this $1 \mathrm{~s}$ assumed to be the relation appropriate for in situ response, the slope of this curve is related to $h$ as 1ndicated following (18), that is

$$
\frac{d \tau_{\text {inc }}}{d \gamma_{\text {Inc }}}=\frac{h+\mu \beta M}{1+(h+\mu \beta M) / G}
$$

A schematic representation of the results for the time variation of postpeak inclusion strain is shown in Figure 7. Also shown in Figure 7 is the decrease of inclusion pore fluid pressure from its value at peak stress. As shown, the decrease is extremely slow until very near instability. In both cases plotted by RR, $p$ has decreased by less than $1 \mathrm{MPa}$ (10 bars) by the time at which instability would have occurred in the absence of pore fluid effects. This result suggests that precursory effects due to the decrease in pore fluid pressure (e.g., a change in wave speed ratios due to vaporization of extsting pore fluid (32)) may be observable only very near to instability.

RR also give a plot of $\theta_{\mathrm{prec}}$ versus the nondimensional strain-rate $\dot{\gamma}_{\infty} t_{D}$. ${ }^{\text {As }}$ in the case of stab1lization by the time dependent stiffness effect, the length of the precursor time Increases with increasing size of the inclusion although agatn the precursor time is not proportional to $a^{2}$. For the same $\tau_{1 n c}$ versus $\gamma_{\text {inc }}$ curve, the precursor times predicted for stabiliation by dilatant hardening are generally greater than 
those predicted for the t1me-dependent stiffness effects. However, RR have suggested that the dilatant hardening calculations may produce upper estimates of the precursor time because the effects of nonlinear fluld compressibility at low pressure were neglected and the values of the dilatancy factor inferred from laboratory results may be much larger than those in situ. Nevertheless, the calculations do Indicate that even very small amounts of dilatancy (too small to cause observable changes in seismlc wave speed travel times) can have a dramatic effect on the evolution of runaway instability.

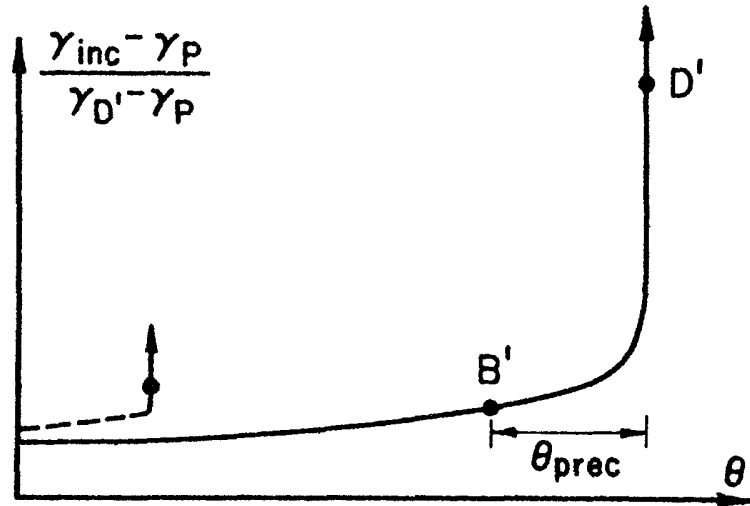

(a)

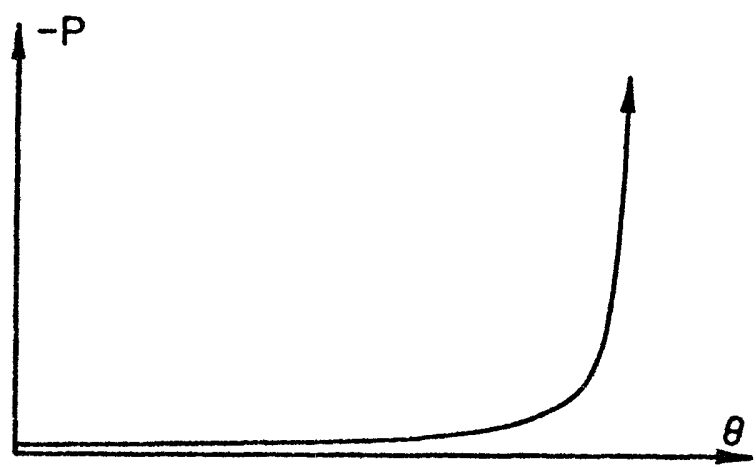

(b)

Figure 7. (a) Typical variation of inclusion strain with $\theta=c t / a^{2}$ as calculated in (9) for stabilization by dilatant hardening effect. Far fleld loading rate is constant. Dashed curve shows drained response (as in Figure 2). Points $B^{\prime}$ and $D^{\prime}$ correspond to points in Figure 4. (b) Decrease in inclusion pore pressure as instability is approached. For numerical values, see (9).

Experiments on Dilatant Hardening. Reynolds (16) has described a number of simple laboratory expertments which illustrate the dilatant hardening of granular materials and Brace and Martin (18) have observed dilatant hardening in axisymetric compression tests on brittle rock. The experiment of Brace and Martin (18) was actually concerned with investigating the "effective stress principle" which, in this context, states that the deviatoric stress at which failure occurs is unaltered by increases of equal amounts in the confining stress and pore fluid pressure. They observed agreement with this principle at low strain-rates but at higher strain-rates they observed a strengthening which they attributed to dilatant hardening. However, the analysts of RR Indicates that the coupling of dilatancy and pore fluid diffusion, in addition to delaying the onset of Instability, or strengthening the rock mass, can set the time scale of the fallure proces3. Recently, Martin (19) has examined more closely this aspect of stabilization by pore fluid effects in axisymetric compression tests on Westerly granite. Martin's (19) results w111 be reviewed here and discussed in the context of the predictions of $R R$.

Martin (19) conducted axisymmetric compression tests on fluid saturated samples of Westerly granite at different strain-rates, confining pressures, and porefluid pressures. Each sample was deformed at constant confining pressure and constant load rate although near instabilfty (within several minutes of fallure) the actual loading rate deviated from the prescribed value because of the inabtlity of the loading apparatus to keep up with the rapid deformation of the sample. During each experlment, the pore fluid pressure in a reservoir attached to one end of the sample was held constant. (of course, the actual pore flutd pressure in the sample will differ from that in the reservoir if dilatancy occurs at a rate faster than pore fluld can diffuse into the sample). Typical results for the time varlation of nominal axtal strain are shown in Figure 8 for different values of the effective confining stress $\bar{\sigma}_{c}$ (confining stress minus pore fluid pressure) and ratios of reservoir pore fluid pressure Prev to confining stress $\sigma_{\text {c }}$. Figure 8 has been replotted from Figure 1 of Martin (19) in terms of the nominal axial strain (axial displacement divided by the sample length $\ell=3.81 \mathrm{~cm}$ ) and time nondimenstonalized by the diffusion time $\ell^{2} / \mathrm{c}$ where $c=0.22 \mathrm{~cm} / \mathrm{sec}$, given by Rice and Cleary (12) for Westerly granite. Martin (19) discusses the results in terms of a qualitative classification of the curves as stable, unstable (Martin uses "dynamic"), or transitional. Curve (a) (for $\bar{\sigma}_{\mathrm{c}}=10^{2} \mathrm{MPa}\left(1 \mathrm{kbar}\right.$ ) and $\mathrm{p}_{\mathrm{rev}} / \sigma_{\mathrm{c}}=0.20$ ) represents unstable behavior: there is an abrupt acceleration of strain terminated by an audible fracture (indicated by the dashed line in Figure 8). Curve (c) (for $\left.\bar{\sigma}_{c}=50 \mathrm{MPa}(500 \mathrm{bars}), p_{\mathrm{rev}} / \sigma_{c}=0.64\right)$ represents stable behavior: there is a relatively long pertod of accelerating strain followed by inaudible fracture. Curve (b) (for $\bar{\sigma}_{c}=10^{2} \mathrm{MPa}$ ( $1 \mathrm{kbar}$ ), $\mathrm{p}_{\mathrm{rev}}$ ' $\left.\sigma_{c}=0.6\right)$ illustrates transitional behavior.

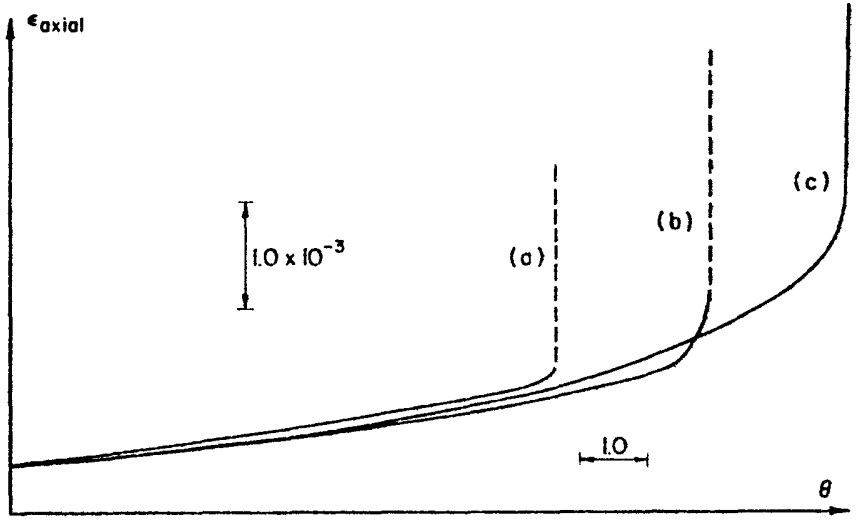

Figure 8. Variation of nominal axial strain $\varepsilon$ axial with $\theta=c t / l^{2}\left(c=0.22 \mathrm{~cm}^{2} / \mathrm{sec}, l=3.81 \mathrm{~cm}\right)$ observed by Martin (19). Redrawn from Figure 1 of (19) so that curves coincide at left hand edge. 
In spite of obvious differences between the experimental configuration and the model analyzed by $R R$, the time variation of nominal axial strain in Figure 8 is qualitatively very similar to the variation of inclusion shear strain predicted by RR. Martin's qualitative classffication of stable or unstable corresponds roughly to the length of the precursor time: short or nonexistent precursor times would be classtfied as unstable; long precursor times correspond to stable behavior (Obviously, the classification depends to some extent on the time scale of interest).

Martin (19) also plots the stress difference at fallure against the ratio $p_{r e v} / \sigma_{c}$ and finds that the transition from unstable to stable behavior occurs at lower values of the stress difference for higher values

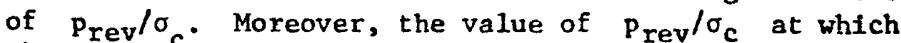
the transition occurs increases with strain-rate. These trends appear to be due to the limit on the pore pressure decrease and, hence, on dilatant hardening, which is imposed by the ambient level of pore fluid pressure in the sample. In the calculations of $R R$, the bulk modulus of the pore fluid was assumed to be constant and consequently the decrease of pore fluld pressure could become unbounded as instability was approached. If, however, effects of nonlinear fluid compressibility had been included, the bulk modulus of the pore fluid $K_{f}$ would vanish when the absolute magnitude of the pore pressure neared zero, that is, when the pore fluid pressure decrease equals in magnitude the ambient value of pore pressure (or, perhaps, well before this point if decreases in pressure cause substantial exsolution of gases). Because the dilatant hardening effect vanishes in the Ilmit as $K_{f}$ goes to zero (see discussion following (48) and (49)), runaway instability would occur at this point and the precursor times would be shorter for decreasing values of the amblent pressure.

\section{Stabillzation of Narrow Inclusion Zones}

The results of $R R$ apply rigorously only for spherical zones. For very narrow zones, the inclusion model may be Inappropriate: the stress concentrations near the ends of the zone may cause the zone to spread. In this case a model of a spreading crack-1tke zone of inelastictty ( $1,2,14$; also, Rice, this volume) may be more suitable. Nevertheless, it is still of interest to consider the inclusion model for narrow zones because, at the very least, the predictions are relevant to assessing conditions for the initiation of narrow spreading zones.

For the stabilization of runaway instability by the time dependent stiffness effect, $R R$ obtained approximate results for narrow inclusion zones simply by using an appropriate value of $\xi$. As mentioned following ( 7$), \xi=10$ for a narrow axisymetric inclusion of aspect ratio $a / b=18$. The predicted time variation of inclusion strain for narrow zones is simflar to that for spherical zones, but RR find that the precursor times for the narrow zone are 10 to 20 times smaller.

Rudnicki (7) has also given a direct plane strain analysis for the stabilization of slip on a frictional surface by the time dependent stiffness effect. He simulated the fault by two edge dislocations of opposite sign with the distance between them chosen so that the relative slip midway between the dislocations is equal to that at the center of a crack of length 2L. Rudnickl (7) obtained an Eshelby relation analogous to (39) by using the plane strain solution of Rice and Cleary (12) for a dislocation suddenly introduced into a Blot soldd (The solution for the spectal case of incompressible solid and fluid constituents was obtained in (13)). The constitutive response was specified as a relation for the shear stress on the fault as a function of the relative slip $\delta$. Qualitatively, the results are simflar to those of the inclusion rodel. Moreover, if the parameters of $\tau$ versus $\gamma$ relation are chosen to be consistent with the $\tau$ versus $\gamma$ relation for a narrow inclusion (Even though, as noted by Rudnicki (7), there seems to be some discrepancy when constitutive parameters estimated from experiments on intact rock are applied to deformation of narrow zones and compared with parameters estinated from frictional sliding experfments) the predictions for the precursor time are in good quantitative agreement. This agreement is in spite of the fact that the calculation of Rudnicki (7) was for plane strain whereas that of $R R$ simulated a narrow axisymetric fault zone. Generally, however, the precursor times predicted by Rudnicki (7) are very short, typically less than a few days fei a plausible range of parameters describing the $\tau$ versus $\delta$ relation, fault lengths of 1 to $5 \mathrm{~km}$., a tectonic stress rate of $10 \mathrm{kPa} /$ year $\left(0, \frac{1}{2} \mathrm{bar} / \mathrm{year}\right)$ and field diffusivities of 0.1 to $1.0 \mathrm{~m}^{2} / \mathrm{sec}$.

One particularly interesting feature of the calculation of Rudnicki (7) for slip on a frictional surface is that the dimensional precursor time tprec can decrease with increasing fault length $L$. Pris result contradicts the expectation that the precursor time should scale roughly as the diffusion time which is proportional to $\mathrm{L} / \mathrm{c}$. Nevertheless, this result can be rationalized by a simple dimensional analysis. The nondimenstonal precursor time $\theta_{\text {prec }}=\mathrm{ct}_{\mathrm{prec}} / \mathrm{L}^{2}$ can be expressed as

$$
\theta_{\text {prec }}=F\left[Q ;(1-v) /\left(1-\nu_{u}\right)\right]
$$

where $Q$ is a nondimensional driving force which is proportional to the far field stress rate $\dot{\tau}_{\infty}$ and may, in addition, be a function of the various nondimensional groups which enter the problem. This dependence can be expressed as

$$
\mathrm{Q}=\mathrm{f}\left[\nu, \nu_{\mathrm{u}}, \dot{\tau}_{\infty} \mathrm{L}^{2} / \mathrm{cG}, \mathrm{L} / \delta^{*}, \Delta \mathrm{t} / \mathrm{G}\right]
$$

where $\Delta \tau$ is the difference between peak and restdual values of friction stress, $\delta^{*}$ is the amount of slip on the fault which is necessary to reduce the shear stress there from the peak value to the residual value, and the remalning quantities have been defined previously. The presence of the nondimensional length $\mathrm{L} / \delta^{*}$ introduces the posstbility that $Q$ will increase faster with $L$ than does the diffusion time. In particular, Rudnicki ( 7 ) assumes a quadratic $\tau$ versus $\delta$ relation and in this case $Q$ has the following form

$$
Q \propto\left(\dot{\tau}_{\infty} L^{2} / C G\right)(\Delta \tau / G)(L / \delta *)^{2}
$$

Because $Q$ is proportional to $\mathrm{L}^{4}$ whereas the diffusion time is proportional to $\mathrm{L}^{2}$, Increasing $\mathrm{L}$ causes the driving force to increase faster than the diffusion time and, consequently, results in a decrease of the precursor time with $L$, at least for a range of paraneters.

As yet, there has been no complete analysis of coupled deformation diffusion effects associated with dilation of a narrow fault zone. Rudnickf (21) (also see (39)) has given some preliminary analysis whtch suggests that the dilatant hardening effect is extremely strong for very narrow zones. This strong effect may, however, be mitigated by the fact that shear of a narrow zone or slip on a surface may be able to generate only a limited anount of dilatancy. In addition to the dilatant hardenting effect, there is also an effect due to the time dependent response of the materfal surroundIng the fault to uplift on the fault surface (This effect did not appear in the analysis of spherical 
Inclustons because the response to hydrostatic stress (see eq. (37)) is the same for drained and undrained conditions). This effect turns ont to be destabilizing in the sense that the effective compressive stress on the fault is less for undrained response than for dralned response. Nevertheless, the preliminary indications are that this effect will be overwhelmed by the stabilizing effect of dilatant hardening.

\section{CONCLUDING DISCUSSION}

This paper has reviewed some predictions for processes preparatory to earthquake faulting based on modeling a fault zone as an embedded inclusion. The model is intended to apply to shallow crustal events for which the rock responds in an essentially brittle fashion and for which the boundary condition at the bottom of the 11thosphere enters only in determining the far fleld stress rate. Because the rock mass has been assumed to be unbounded, the analysis applies strictly to earthquake events which are small enough that effects of the free surface are not significant.

For fault zone material which is straln-softening, as is typical of brittle rock, the model predicts that runaway instability w111 be preceded by an acceleration of Inclusion shear strain. If the deformation of the rock mass is coupled to the diffusion of an infiltrating pore fluid, then instability can be delayed for a length of time, which is defined as the precursor time, and the acceleration of strain prior to instability is more pronounced.

The precursory effects described here are shortterm, on the time scale of days rather than years. For a spherical inclusion zone of radius $1 \mathrm{~km}$., RR give 2 to 10 days as a minimum estimate of the time pertod prior to instability during which precursory effects are likely to be detectable. For very narrow zones, this time period will be shorter although in this case, a spreading crack-like zone of inelasticity is probably a more appropriate model than an inclusion zone.

The precursory acceleration of incluston shear strain may be detectable as an increased rate of deformation (tilt or strain) of the ground surface although the magnitude of the effect will decrease roughly as the Inverse square of distance from the fault zone. Changes in physical properties due to the microcracking which accompantes the inelastic straining may provide more easily detectable precursors. The calculations of RR suggest that relatively large amounts of dilatancy are needed to cause changes in seismic propertfes by the undersaturation of fluid-infiltrated rock. However, transport properties may be affected by smaller amounts of microcracking causing changes in restivity (40) or radon content of wells (41). Moreover, resistivity changes may be augmented by the flow of pore flutd induced by deformation of the inclusion zone.

The mode 1 which has been reviewed here is a simple one and more elaborate models could be constructed. Coupled deformation diffusion effects have been analyzed precisely only for spherical inclusions and the effects of geometry could be considered more carefully. A full analysis of the effect of localization of deformation on the subsequent response of the inclusion has not yet been given. It may be that localization of deformation inftiates a spreading crack-like zone of inelasticity, prior to the onset of runaway, rather than simply modifylng the bulk response of the inclusion material as assumed by $R R$. In any case, the results of Rice and Simons (14) for stabilization of a spreading fault by the time dependent stiffness effect are promising and this is an area that would seem to merit more attention. However, at present, the most pressing need seems to be for more accurate values of material and transport properties, particularly values appropriate to in situ behavior.

\section{ACKNOWLEDGEMENT}

Support during the preparation of this review was provided by the U.S. Geological Survey National Earthquake Hazards Reduction Program and by the Department of Theoretical and Applied Mechantes at the Untversity of Illinois.

\section{REFERENCES}

1. Rice, J. R., "Theory of Precursory Processes In the Inception of Earthquake Rupture," Gerländs Beitr. Geophysics, Vol. 88, 1979, pp. 91-127.

2. Rice, J. R., "The Mechantes of Earthquake Rupture," Proceedings of the International School of Physics "Enrico Fermi", Italian Physical Soctety, Course LXXVIII (1979, Varenna on Lake Como, Italy) on Physics of the Earth's Interlor, edited by E. Boscht, North-Holland Publishing Co., publication expected, 1980-81.

3. Rudnick1, J. W., "The Inception of Faulting in a Rock Mass With a Weakened Zone," Journal of Geophystcal Research, Vol. 82, 1977, pp. 844-854.

4. Stuart, W. D., "Strain Softening Instability Model for the San Fernando Earthquake," Science, Vol. 203, 1979, pp. 907-910.

5. Stuart, W. D., "Strain Softening Prior to TwoDimensional Strike-S1ip Earthquakes," Journal of Geophysical Research, Vo1. 84, 1979, pp. 1063-1070. 6. Stuart, W. D. and Mavko, G. M., "Earthquake Instability on a Strike-Slip Fault," Journal of Geophysical Research, Vol. 84, 1979, pp. 2153-2160.

7. Rudnicki, J. W., "The Stabilization of Slip on a Narrow Weakening Fault Zone by Coupled Deformation Pore Fluid Diffusion," Bulletin of the Seismological Society of America, Vol. 69, 1979, Pp. 1011-1026.

8. Rice, J. R., Rudnick1, J. W., and Stmons, D. A "Deformation of Spherical Cavities and Inclusions in F1u1d-Inf1ltrated Elastic Materlals," International Journal of Solids and Structures, Vol. 14, 1978, pp. 289-303.

9. Rice, J. R. and Rudnick1, J. W., "Earthquake Precursory Effects Due to Pore Fluid Stabilization of a Weakening Fau1t Zone," Journal of Geophysical Research, Vo1. 84, 1979, pp. 2177-2193.

10. Terzaghi, K. and Peck, R. B., Soll Mechanics in Engineerling Practice, 2nd Edition, Wiley, New York 1967.

11. Biot, M. A., "General Theory of Three Dimensional Consolidation," Journal of Applied Physics, Vol. 12, 1941, pp. 155-164.

12. Rice, J. R. and Cleary, M. P., "Some Basic Stress Diffusion Solutions for Fluld-saturated Elastic Porous Media With Compressible Constituents," Reviews of Geophysics and Space Physics, Vol. 14, 1976, pp. 227-241.

13. Booker, J. R., "Time-dependent Strain Following Faulting of a Porous Medium," Journal of Geophysica: Research, Vo1. 79, 1974, pp. 2037-2044.

14. Rice, J. R. and Stmons, D. A., "The Stabiliza. tion of Spreading Shear Faults by Coupled DeformationDiffusion Effects in Fluid-Infiltrated Porous Materials Journal of Geophysical Research, Vol. 81, 1976, pp. 5322-5334.

15. Rulna, A., "Influence of Coupled DefomationDiffusion Effects on Retardation of Hydraulic Fracture, In Proceedings of the 19th U.S. Sympostum on Rock Mechanics, Stateline, Nevada, edited by Y. S. Kim, 1978 pp. 274-282.

16. Reynolds, 0., "On the Dilatancy of Media Composed of Rigid Particles In Contact, With Experimental Illustrations," Philosophical Magazine, 1885. (Reprinted in Papers on Mechanical and Physical Subjects by 0 . Reynolds, Vol. 2, Cambridge University Press, New York, 1901, pp. 203-216. 
17. Frank, F. C., "On Dilatancy in Relation to Seismic Sources," Revlews of Geophysics and Space Physics, Vol. 3., 1964, pp. 485-503.

18. Brace, W. F. and Martin, R. J., "A Test of the Law of Effective Stress for Crystalline Rocks of Low Porosity," International Journal of Rock Mechanics and Mining Sclences, Vol. 5, 1968, pp. 415-426.

19. Martin, R. J., "Pore Pressure Stabilization of Fallure in Westerly Grantte," Geophysical Research Letters, Vol. 7, 1980, in press.

20. Rice, J. R., "On the Stability of Dilatant Hardening for Saturated Rock Masses," Journal of Geophysical Research, Vo1. 80, 1975, pp. 1531-1536.

21. Rudnicki, J. W., "Localization of Deformation, Brittle Rock Fallure, and Model for the Inception of Earth Faulting," Ph.D. Thesis, Brown University, Providence, RI, 1977.

22. Eshelby, J. D., "The Determination of the Elastic Field of an Ellipsoidal Inclusion and Related Problems," Proceedings of the Royal Society of London, Series A, Vol. 241, 1957, pp. 376-396.

23. Eshelby, J. D., "Elastic Inclusions and Inhomogeneities," in Progress in Solid Mechanics, edited by I. N. Sneddon and R. Hill, Vol. 2, North Ho1land, Amsterdam, 1961, pp. 88-140.

24. Prescott, W. H. and Savage, J. C., "Straln Accumulation on the San Andreas Fault Near Palmdale, California," Journal of Geophysical Research, Vol. 81, 1976, pp. 4901-4908.

25. Savage, J. C. and Prescott, W. H., "Geodimeter Measurements of Strain During the Southern Calffornia Uplift," Journal of Geophysical Research, Vo1. 84, 1979, pp. 171-177.

26. Rudnicki, J. W. and Rice, J. R., "Conditions for the Localization of Deformation in Pressuresensitive Dilatant Materials," Journal of the Mechanics and Physics of Solids, Vo1. 23, 1975, pp. 371-394. 27. Cleary, M. P. and Rudnicki, J. W., "The Initiation and Propagation of Dilatant Rupture Zones in Geological Materials," in The Effects of Volds on Material Deformation, edited by S. C. Cowin, Am. Soc. Mech. Engr. App1. Mech. Division, Vol. 16, New York, 1976, pp. 13-30.

28. Walsh, J. B., "Stiffness in Faulting and in Friction Experiments," Journal of Geophyslcal Research, Vol. 76, 1971, pp. 8597-8598.

29. Cleary, M. P., "Heterogenelty and Porosity Effects on the Response of Geomaterials," in Advances in Civil Engineering Through Engineering Mechanics, American Soctety of Civil Engineers, New York, 1977, pp. $360-363$.

30. O'Conne11, R. J. and Budiansky, B., "ViscoElastic Properties of Fluid-saturated cracked solids," Journal of Geophys1cal Research, Vol. 82, 1977, pp. 5719-5735.

31. Nur, A. and Byerlee, J. D., "An Exact Effective Stress Law for Elastic Deformation of Rock With Fluid," Journal of Geophysical Research, Vol. 76, 1971, pp. 6414-6419.

32. Anderson, D. L. and Whitcomb, J. H., "Timedependent Seismology," Journal of Geophysical Research, Vol. 80,1975 , pp. 718-732.

33. Kovach, R. L., Nur, A., Wesson, R. L. and Robinson, R., "Water-level Fluctuations and Earthquakes on the San Andreas Fault Zone," Geology, Vol. 3, 1975, pp. 437-440.

34. Rice, J. R. and Simons, D. A., "Fundamentals of Deformation and Rupture Processes in Porous Geological Materials, Summary of Work on U.S.G.S. contract 14-68-0001-15866 Mod.1 at Brown University," in Summary of Technical Reports of Participants in National Earthquake Hazards Reduction Program, Vol. 4, U.S. Geological Survey, Office of Earthquake Studies, Menlo Park, CA., December, 1978, p. 500.
35. O'Connell, R. J. and Budiansky, B., "Se1smic Velocfties in Dry and Saturated Cracked Solids," Journal of Geophystcal Research, Vo1. 79, 1974, pp. 54125426.

36. Carrol1, M. M., "An Effective Stress Law for Anisotropic Elastic Deformation," Journal of Geophysical Research, Vo1. 84, 1979, pp. 7510-7512.

37. Rice, J. R., "Pore Pressure Effects in Inelastic Constttutive Formulations for Fissured Rock Masses," in Advances in Civil Engineering Through Engtneering Mechanics, American Society of Civil Engineers, New York, 1977, pp. 360-363.

38. Blot, M. A., "Nonlinear and Semllinear Rheology of Porous Solids," Journal of Geophysical Research, Vol. 78, 1973, pp. 4924-4937.

39. Minster, J. B. and Rudnicki, J. W., "Theoretical Studies in the Role of Pore Fluid in Premonitory Phenomena," Final Technical Report on U.S. Geological Survey, Contract No, 14-08-0001-16795 to California Institute of Technology, June, 1979.

40. Mazella, A. and Morrison, H. F., "Electrical Resistivity Variations Associated With Earthquakes on the San Andrea Fault," Sclence, Vol. 185, 1974, pp. 855-857.

41. Wak1ta, H., Nakamura, Y., Notsu, K., Noguchi, M., Asada, T., "Radon Anomaly: A Possible Precursor of the 1978 Izu-Oshima-kınka1 Earthquake,"Science, Vol. 207, 1980, pp. 882-883. 


\section{On Singular Solutions for Linear \\ Fluid Infiltrated Elastic Porous Solids}

$$
\text { by }
$$

John W. Rudnicki

\footnotetext{
Department of Theoretical and Applied Mechanics University of Illinois

Urbana, IL 61801 July, 1980
} 


\section{Abstract}

This note corrects expressions given by Cleary (1977) for fluid mass source and dipole solutions in linear fluid infiltrated porous elastic solids. The correspondence between point force and fluid mass source solutions, which because of an algebraic error in Cleary's analysis is only partially revealed there, is fully exposed here. In particular, the stress and displacement fields due to a suddenly applied point force are demonstrated to comprise a time independent portion, which is the classical elastic solution based on the undrained (shorttime) moduli and a time dependent portion, which is the solution for a continuous fluid mass dipole. A corollary is that the time dependent functions entering the point force solution can be obtained from a single function which enters the solution for a fluid mass source. 


\section{Introduction}

Although the response of near surface earth materials is seldom likely to be precisely linear, the theory of linear fluid infiltrated elastic solids has proven to be very useful in studying a variety of geotechnical and geophysical problems. These include soil consolidation [1, 2], hydraulic fracture [3, 4], and various aspects of earth faulting $[3,5,6,7,8,9,10]$.

The governing equations of a linear fluid infiltrated elastic porous solid are precisely the same as those for the fully coupled theory of linear thermoelasticity. However, the coupling which can be justifiably neglected in thermoelasticity must be retained in the porous media equations. This coupling greatly magnifies the difficulty of analysis and, as a consequence, there are a very limited number of analytical solutions for these equations. This situation was greatly improved by Cleary [11] who, in a elegant and extensive analysis, established the three dimensional fundamental solutions and outlined their use in modelling embedded regions of inelasticity. However, as a result of an algebraic error in the expressions given by cleary [11] for the continuous mass source solution, the full extent of the correspondence between point force and fluid mass source solutions is not revealed. This note fully exposes this correspondence and in so doing corrects the expressions given by cleary [11] and tabulates them in an alternate, more compact form. It is hoped that these results will facilitate the implementation of Cleary's solutions to more complex boundary value problems.

\section{Review of Governing Equations}

The most direct formulation of the governing equations for a linear fluidinfiltrated porous elastic solid, which were first established by Biot [2], has been given by Rice and Cleary [3] and this treatment will be followed here. If the deformation occurs so slowly that the infiltrating fluid mass has sufficient 
time to diffuse from material elements, there will be no alteration of pore fluid pressure. In this limit of longtime deformation, the response is labelled drained and the total stress $\sigma_{i j}$ is related to the displacement gradient $u_{i, j}\left(=\partial u_{i} / \partial x_{j}\right)$ as in an ordinary linear elastic solid:

$$
\sigma_{i j}=\mu\left(u_{i, j}+u_{j, i}\right)+\lambda u_{k, k} \delta_{i j}
$$

where $\lambda$ and $\mu$ are the Lame moduli appropriate for drained response and $\delta_{i j}$ is the Kronecker delta. More generally, a term proportional to the pore fluid pressure must be included in (1):

$$
\sigma_{i j}=\mu\left(u_{i, j}+u_{j, i}\right)+\lambda u_{k, k} \delta_{i j}-\zeta p \delta_{i j}
$$

where $\zeta=1-K_{S}^{\prime}, K(=\lambda+2 \mu / 3)$ is the drained bulk modulus and $K_{S}^{\prime}$ is an empirical constant which, under circumstances stated precisely by Rice and Cleary [3], can be identified with the bulk modulus of the solid constituents. A second constitutive relation is needed for the alteration in $\mathrm{m}$, the fluid mass content per unit volume of porous solid:

$$
m-m_{o}=\zeta \rho_{o}\left[u_{k, k}+\zeta p /\left(\lambda_{u}-\lambda\right)\right]
$$

where $m_{0}$ is the reference value of $m, \rho_{0}$ is the mass density of homogeneous pore fluid and $\lambda_{\mathrm{u}}$ is the Lame modulus for undrained response. Undrained response occurs when load alterations are too rapid to allow time for fluid mass diffusion from material elements. Hence, $m=m_{0}$ for undrained response and solving for $p$ from (3) and substituting into (2) recovers the form of (1) with $\lambda$ replaced by $\lambda_{\mathbf{u}}$. These constants satisfy $\lambda<\lambda_{\mathbf{u}}<\infty$ where the corresponding relation in terms of Poisson's ratio $v(=\lambda / 2(\lambda+\mu))$ is $\nu<v_{\mathrm{u}}<1 / 2$. In both relations the upper limit is attained for separately incompressible 
constituents and the lower for highly compressible pore fluid. Setting $\mathrm{m}^{=} \mathrm{m}_{\mathrm{o}}$ in (3) and using (2) to eliminate $u_{k, k}$ reveals that for undrained response the alteration of pore fluid pressure is given by

$$
\mathrm{p}=-\mathrm{B} \sigma_{\mathrm{kk}} / 3
$$

where $B=\left(\lambda_{u}-\lambda\right) / \zeta\left(\lambda_{u}+2 \mu / 3\right)$ is Skempton's pore pressure coefficient [3]. The constitutive formulation is completed by Darcy's law which, in the absence of body force, is given by

$$
q_{i}=-\rho_{0} \kappa \partial p / \partial x_{i}
$$

where $q_{i}$ is the mass flow rate per unit area in the $x_{i}$ direction and $k$ is a permeability. The permeability is often expressed as $k=k / \gamma$ where $k$ is measured in units of area and $\gamma$ is the fluid viscosity.

The constitutive equations (2), (3), and (5) must be combined with field equations expressing equilibrium and fluid mass conservation. In the absence of body forces, the equilibrium equation is

$$
\sigma_{i j, i}=0
$$

or, if (2) is substituted into this equation, it becomes

$$
(\lambda+\mu) u_{i, i j}+\mu \nabla^{2} u_{j}-\zeta p, j=0
$$

where $\nabla^{2}(. .)=.\partial^{2}(. ..) / \partial x_{k} \partial x_{k}$. Another useful equation can be obtained by forming the divergence of (7). The result is

$$
\nabla^{2}\left[(\lambda+2 \mu) u_{k, k}-\zeta p\right]=0
$$


The equation of fluid mass conservation is

$$
\underset{\sim}{\nabla} \underset{\sim}{q}+\partial m / \partial t=0
$$

where $\underset{\sim}{\nabla} \cdot \underset{\sim}{q}=q_{k, k}$. Substituting Darcy's law (5) into (9) and using (3) and (8) yield a homogeneous diffusion equation for $m$ [3],

$$
c \nabla^{2} \mathrm{~m}=\partial \mathrm{m} / \partial t
$$

where the diffusivity $c$ can be expressed as follows

$$
\mathbf{c}=\kappa \zeta^{-2}\left(\lambda_{\mathbf{u}}-\lambda\right)\left[(\lambda+2 \mu) /\left(\lambda_{\mathbf{u}}+2 \mu\right)\right]
$$

\section{Point Force Solution}

The point force solution given by Cleary [11] will be outlined here for later comparison with the corrected expressions for the fluid mass dipole solutions. In addition, the influence functions will be written in a form which is somewhat more compact than that given by Cleary.

Consider a point force $\mathrm{P}_{\mathrm{k}}$ to be suddenly applied at the origin at $t=0$. This point force may, of course, be regarded as a body force $\mathrm{P}_{\mathrm{k}} \delta(\mathrm{x}) \mathrm{H}(\mathrm{t})$ entering (6) where $\delta(x)$ is the three dimensional Dirac delta function and $H(t)$ is the unit step function. Cleary's [11] construction of the solution begins by recognizing that linearity, isotropy, and dimensional analysis require the resulting pore pressure response to have the following form

$$
p(\underset{\sim}{x}, t)=\frac{P_{k} x_{k}}{r^{3}} g_{1}(r / \sqrt{c t})
$$

where $r=\left(x_{k} x_{k}\right)^{1 / 2}$ and $g_{1}$ (Cleary used $f_{1}$ ) is to be determined. Note that the argument of $g_{1}$ is dimensionless and because drained conditions will be 
attained at $t=\infty, g_{1}(0)=0$. Cleary [11] uses the same considerations to deduce the form of the resulting displacement field

$$
\mu_{i}(\underset{\sim}{x}, t)=\frac{1}{8 \pi r}\left\{\frac{x_{i} x_{j}}{r^{2}} \frac{(\lambda+\mu)}{(\lambda+2 \mu)} g_{2}(\xi)+\delta_{i j} \frac{(\lambda+3 \mu)}{(\lambda+2 \mu)} g_{3}(\xi)\right\} P_{j}
$$

where $g_{2}$ and $g_{3}$ are to be determined and the abbreviation $\xi=r /(c t)^{1 / 2}$ has been introduced. As $t \rightarrow \infty$, the expression (12) must reduce to the classical. elasticity solution based on the drained moduli and, consequently, the coefficients have been chosen such that $g_{2}(\xi=0)=g_{3}(\xi=0)=1$. The functions $f_{2}$ and $\mathrm{f}_{3}$ used by Cleary [11] are related to $g_{2}$ and $g_{3}$

$$
\begin{aligned}
& \mathrm{f}_{2}=[(\lambda+\mu) / 8 \pi(\lambda+2 \mu)] \mathrm{g}_{2} \\
& \mathrm{f}_{3}=[(\lambda+3 \mu) / 8 \pi(\lambda+2 \mu)] \mathrm{g}_{3} .
\end{aligned}
$$

Substituting (11) and (12) into (2) yields the form of the stresses

$$
\begin{aligned}
4 \pi r^{2} \sigma_{i j}= & \frac{\mu}{(\lambda+2 \mu)}\left[\frac{P_{k} x_{k}}{r} \sigma_{i j} G_{3}(\xi)-\frac{P_{i} x_{j}+x_{j} P_{i}}{r} G_{2}(\xi)\right] \\
& -\frac{3(\lambda+\mu)}{(\lambda+2 \mu)} \frac{x_{i} x_{j}}{r^{2}} \frac{x_{k} P_{k}}{r} G_{1}(\xi)
\end{aligned}
$$

where

$$
\begin{aligned}
& 3 G_{1}(\xi)=-\xi^{4} \frac{d}{d \xi}\left(\xi^{-3} g_{2}\right) \\
& 2 \mu G_{2}(\xi)=-(\lambda+3 \mu) \xi^{2} \frac{d}{d \xi}\left(\xi^{-1} g_{3}\right)-(\lambda+\mu) g_{2}
\end{aligned}
$$

and 


$$
\begin{aligned}
2 \mu G_{3}(\xi)= & \mu^{-1}\left[(\lambda+\mu)(\lambda+2 \mu) g_{2}+\lambda(\lambda+\mu) \xi g_{2}^{\prime}+\lambda(\lambda+3 \mu)\left(\xi g_{3}^{\prime}-g_{3}\right)\right] \\
& -8 \pi \zeta(\lambda+2 \mu) g_{1}
\end{aligned}
$$

where (... $)^{\prime}=\mathrm{d}(. ..) / \mathrm{d} \xi$. The functions $\mathrm{G}_{1}, \mathrm{G}_{2}$, and $\mathrm{G}_{3}$ equal unity for $\xi=0(t=\infty)$ and they differ by constant factors from $F_{1}\left(=-3(\lambda+\mu) G_{1} / 4 \pi(\lambda+2 \mu)\right), F_{2}\left(=-\mu G_{2} / 4 \pi(\lambda+2 \mu)\right)$, and $\mathrm{F}_{3}\left(=\mu G_{3} / 4 \pi(\lambda+2 \mu)\right)$ used by Cleary.

Solution then follows from the procedure discussed in detail by cleary [11]: essentially, (6), (8), and (10) are used to obtain ordinary differential equations for $G_{1}, G_{2}, G_{3}$, and $g_{1}$ which can be solved to determine these functions up to a constant term; (14a) and (14b) are then used to determine $g_{2}$ and $g_{3}$ and (14c) yields the value of the constant.

The resulting expressions for $G_{1}, G_{2}$, and $G_{3}$ can be written in compact form as follows:

$$
\begin{aligned}
& G_{1}(\xi)=1+\frac{\mu\left(\lambda_{u}-\lambda\right)}{\left(\lambda_{u}+2 \mu\right)(\lambda+\mu)}\left[h_{1}(\xi)-\frac{5}{3} h_{2}(\xi)\right] \\
& G_{2}(\xi)=1-\frac{\left(\lambda_{u}-\lambda\right)}{\left(\lambda_{u}+2 \mu\right)}\left[h_{1}(\xi)-h_{2}(\xi)\right] \\
& G_{3}(\xi)=1-\frac{\left(\lambda_{u}-\lambda\right)}{\left(\lambda_{u}+2 \mu\right)}\left[h_{1}(\xi)+h_{2}(\xi)\right]
\end{aligned}
$$

where 


$$
\begin{aligned}
& h_{1}(\xi)=\frac{1}{2 \sqrt{\pi}} \int_{0}^{\xi} n^{2} \mathrm{e}^{-\eta^{2} / 4} \mathrm{~d} n=\operatorname{erf}(\xi / 2)-\xi \mathrm{e}^{-\xi^{2} / 4} / \sqrt{\pi} \\
& \mathrm{h}_{2}(\xi)=\frac{\xi^{-2}}{2 \sqrt{\pi}} \int_{0}^{\xi} n^{4} \mathrm{e}^{-\eta^{2} / 4} \mathrm{~d} \eta=6 \xi^{-2} \mathrm{~h}_{1}(\xi)-\xi \mathrm{e}^{-\xi^{2} / 4 / \sqrt{\pi}} \\
& \text { and } \operatorname{erf}(\mathrm{z})=(2 / \sqrt{\pi}) \int_{0}^{z} \mathrm{e}^{-\mathrm{x}^{2}} \mathrm{dx}
\end{aligned}
$$

The function $h_{1}(\xi)$ is zero at $\xi=0(t=\infty)$ and unity at $\xi=\infty(t=0)$ whereas $h_{2}(\xi)$ vanishes at both limits.

The functions $g_{1}, g_{2}$, and $g_{3}$ can be written as follows:

$$
\begin{aligned}
& g_{1}(\xi)=\left[\left(\lambda_{u}-\lambda\right) / 4 \pi \zeta\left(\lambda_{u}+2 \mu\right)\right] h_{1}(\xi) \\
& g_{2}(\xi)=1+\frac{\mu\left(\lambda_{u}-\lambda\right)}{\left(\lambda_{u}+2 \mu\right)(\lambda+\mu)}\left[h_{1}(\xi)-h_{2}(\xi)\right] \\
& g_{3}(\xi)=1-\frac{\mu\left(\lambda_{u}-\lambda\right)}{\left(\lambda_{u}+2 \mu\right)(\lambda+3 \mu)}\left[h_{1}(\xi)-\frac{1}{3} h_{2}(\xi)+(2 / 3 \sqrt{\pi}) \xi e^{-\xi^{2} / 4}\right]
\end{aligned}
$$

(The expression given for $\mathrm{f}_{3}$ by Cleary [11] in his equation (31) contains an extra factor of 2 in the coefficient of $\xi \exp \left(-\xi^{2} / 4\right)$ but the preceding expression in his eq. (29) is correct.)

\section{Fluid Mass Source}

To obtain the solutions for fluid mass sources, Cleary [11] used the procedure of Rice and Cleary [3] for spherically symmetric problems. However, a slightly different approach, analogous to that used by Cleary to obtain the point 
force solution, is used here.

The fundamental source solution of (10) corresponding to the instantaneous injection of an amount $Q$ of fluid mass at the origin at $t=0$ is well known to be $(e \cdot g \cdot[12])$

$$
m(\underset{\sim}{x}, t)=\frac{Q}{(4 \pi c t)^{3 / 2}} \exp \left(-r^{2} / 4 c t\right)
$$

The corresponding displacement field is spherically symmetric and is required by dimensional analysis, linearity, and isotropy to have the following form:

$$
u_{i}(\underset{\sim}{x}, t)=\frac{Q \underset{i}{x_{0}} U(r / \sqrt{c t})}{\rho_{0}}
$$

where the dimensionless function $U$ can be determined by substitution of (17) and (18) into (7) after using (3) to eliminate $\mathrm{p}$ in favor of $\mathrm{m}-\mathrm{m}_{\mathrm{o}}$. Solution of the resulting equation yields

$$
U(\xi)=\left[\left(\lambda_{u}-\lambda\right) / 4 \pi \zeta\left(\lambda_{u}+2 \mu\right)\right] h_{1}(\xi)
$$

where $h_{1}(\xi)$ has been given following (15). The pore pressure can be determined from (3) and the result is

$$
p(\underset{\sim}{x}, t)=\frac{\left(\lambda_{u}-\lambda\right)(\lambda+2 \mu)}{\zeta^{2}\left(\lambda_{u}+2 \mu\right)} \frac{Q}{\rho_{o}} \frac{\exp \left(-r^{2} / 4 c t\right)}{(4 \pi c t)^{3 / 2}}
$$

The stress computed from (2) is

$$
\sigma_{i j}=\frac{Q}{\rho_{0}} \frac{\mu\left(\lambda_{u}-\lambda\right)}{2 \pi r^{3} \zeta\left(\lambda_{u}+2 \mu\right)}\left[\delta_{i j}\left(h_{1}-\xi h_{1}^{\prime}\right)+\frac{x_{i} x_{j}}{r^{2}}\left(\xi h_{1}^{\prime}-3 h_{1}\right)\right]
$$


where the prime again denotes differentiation with respect to the argument.

The solutions for continuous injection of fluid mass at a constant rate $q$ can be obtained by the usual superposition procedure: replace $Q$ by $q \mathrm{dt}^{\prime}$ and $t$ by $t-t^{\prime}$ in (17) and (18) and integrate from $t^{\prime}=0$ to $t^{\prime}=t$. The results are as follows:

$$
m(x, t)=(q / 4 \pi c r) \operatorname{erfc}(\xi / 2)
$$

where $\operatorname{erfc}(z)=1-\operatorname{erf}(z)$ and

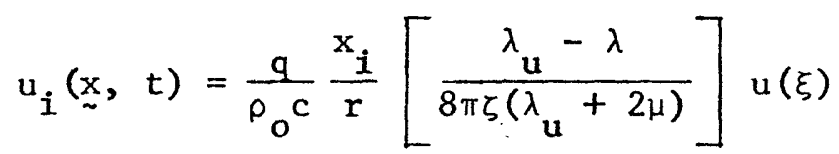

where

$$
u(\xi)=\operatorname{erfc}(\xi / 2)+2 \xi^{-2} h_{1}(\xi)
$$

The pore fluid pressure and stress can then be calculated from (3) and (2). These are

$$
p(\underset{\sim}{x}, t)=\frac{q}{\rho_{0} c} \frac{1}{4 \pi r}\left[\frac{\left(\lambda_{u}-\lambda\right)(\lambda+2 \mu)}{\zeta^{2}\left(\lambda_{u}+2 \mu\right)}\right] \operatorname{erfc}(\xi / 2)
$$

and

$$
\begin{aligned}
\sigma_{i j}= & -\frac{q}{\rho_{o} c} \frac{\left(\lambda_{u}-\lambda\right) \mu}{4 \pi \zeta\left(\lambda_{u}+2 \mu\right)}\left\{\frac{\delta_{i j}}{r}\left[\operatorname{erfc}(\xi / 2)-2 \xi^{-2} h_{1}(\xi)\right]\right. \\
& \left.+\frac{x_{i} x_{j}}{r^{3}}\left[\operatorname{erfc}(\xi / 2)+6 \xi^{-2} h_{1}(\xi)\right]\right\} .
\end{aligned}
$$


The last expression (26) corrects that given in eqn. (39b) of cleary [11]. As mentioned earlier, Cleary [11] solved for the stresses directly by using the procedure of Rice and Cleary [3] for spherically symmetric problems. The stress $\sigma_{r r}$ is given correctly in Cleary's eqn. (39a) but he omits a factor of one-half in the first term of the expression for $\sigma_{\theta \theta}$. Consequently, the cartesian coordinate form of the stress given in eqn. (39b) is incorrect and, because this expression is used to obtain the stress field of a fluid mass dipole, the dipole stress field given in Cleary's [11] eqn. (43) is also incorrect. The correct expression is given in the next section.

\section{Fluid Mass Dipoles}

The solution for fluid mass dipoles can be obtained from the source solutions of the last section by the usual technique: if a particular field quantity is given by $\mathrm{qF}(\underset{\sim}{\mathrm{x}}, t)$ for a source of strength $\mathrm{q}$, then the corresponding quantity for a dipole of strength $q$ and direction $h_{\alpha}$ is given by

$$
F_{\text {dipole }}(\underset{\sim}{x}, t)=-q h_{\alpha} \frac{\partial}{\partial x_{\alpha}} F(\underset{\sim}{x}, t)
$$

Applying this procedure to the fluid mass solution for a continuous source given by (22) yields

$$
m(\underset{\sim}{x}, t)=\frac{q_{\alpha}}{4 \pi c} \frac{x_{\alpha}}{\dot{r}^{3}}\left[1-h_{1}(\xi)\right]
$$

where $\mathrm{q}_{\alpha}=\mathrm{qh}_{\alpha}$. Similarly, the solutions for the pore fluid pressure, displacement, and stress follow from (24), (25), and (26) respectively. These are recorded below: 


$$
\begin{aligned}
p(\underset{\sim}{x}, t)= & \frac{q_{\alpha}}{\rho_{o} c}\left[\frac{\left(\lambda_{u}-\lambda\right)(\lambda+2 \mu)}{4 \pi \zeta^{2}\left(\lambda_{u}+2 \mu\right)}\right] \frac{x_{\alpha}}{r^{3}}\left[1-h_{1}(\xi)\right] \\
u_{i}(\underset{\sim}{x}, t)= & -\frac{q_{\alpha}}{\rho_{0} c}\left[\frac{\left(\lambda_{u}-\lambda\right)}{8 \pi \zeta\left(\lambda_{u}+2 \mu\right)}\right] \frac{1}{r}\left\{\delta_{i \alpha} u(\xi)+\right. \\
& \left.+\frac{x_{i} x_{\alpha}}{r^{2}}\left[\xi u^{\prime}(\xi)-u(\xi)\right]\right\}
\end{aligned}
$$

where $u(\xi)$ is given by (24); and

$$
\begin{aligned}
\sigma_{i j}\left(x_{\sim}^{x}, t\right)= & \frac{q_{\alpha}}{\rho_{o} c}\left[\frac{\left(\lambda_{u}-\lambda\right) \mu}{4 \pi \zeta\left(\lambda_{u}+2 \mu\right)}\right] \frac{1}{3}\left\{x_{\alpha} \delta_{i j}\left(\xi \Sigma_{1}^{\prime}-\Sigma_{1}\right)+\right. \\
& \left.+\left(\delta_{i \alpha} x_{j}+\delta_{j \alpha} x_{i}\right) \Sigma_{2}(\xi)-3 \frac{x_{i} x_{j} x_{\alpha}}{r^{2}}\left(\Sigma_{2}-\frac{1}{3} \xi \Sigma_{2}^{\prime}\right)\right\}
\end{aligned}
$$

where

$$
\begin{aligned}
& \Sigma_{1}(\xi)=\operatorname{erfc}(\xi / 2)-2 \xi^{-2} h_{1}(\xi) \\
& \Sigma_{2}(\xi)=\operatorname{erfc}(\xi / 2)+6 \xi^{-2} h_{1}(\xi)
\end{aligned}
$$

Alternatively, the functions $\Sigma_{1}$ and $\Sigma_{2}$ can be expressed as follows in terms of $u(\xi)$ :

$$
\Sigma_{1}(\xi)=u(\xi)+\xi u^{\prime}(\xi)
$$

and

$$
\Sigma_{2}(\xi)=u(\xi)-\xi u^{\prime}(\xi)
$$


As noted by Cleary [11], the time dependence of the pore fluid pressure $p$ (29) bears an obvious resemblance to the pore pressure field of a suddenly applied point force ((11) with (16a)). Specifically, the value of p in (29) minus its long-time value $\left(t=\infty, \xi=0, h_{1}(\xi=0)=0\right)$ is the pore fluid pressure due to a point force with components given by

$$
P_{\alpha}=q_{\alpha}(\lambda+2 \mu) / \rho_{o} \zeta c
$$

However, because of his error in computing the continuous fluid source stress field and hence the dipole stress field, Cleary [11] did not recognize that the dipole stress and displacement fields bear a similar relationship to the point force solution. In particular, (30) can, after some rearrangement, be rewritten as

$$
\begin{aligned}
\mu_{i}= & \frac{q_{\alpha}}{\rho_{0} c \zeta} \frac{(\lambda+2 \mu)}{8 \pi r}\left\{\delta_{i \alpha}\left[\frac{\left(\lambda_{u}+3 \mu\right)}{\left(\lambda_{u}+2 \mu\right)}-\frac{(\lambda+3 \mu)}{(\lambda+2 \mu)} g_{3}(\xi)\right]+\right. \\
& \left.+\frac{x_{i} x_{\alpha}}{r^{2}}\left[\frac{\left(\lambda_{u}+\mu\right)}{\left(\lambda_{u}+2 \mu\right)}-\frac{(\lambda+\mu)}{(\lambda+2 \mu)} g_{2}(\xi)\right]\right\}
\end{aligned}
$$

This can be recognized as the difference between the time-independent classical elasticity solution based on the undrained moduli for a point force given by (32) and the porous media solution (12) for the same point force. Conversely, the solution (12) for a suddenly applied point force in a fluid infiltrated porous medium comprises the difference between the classical elasticity solution based on the undrained moduli and the response to a continuous fluid mass dipole whose components are given by solving (32) for $\mathrm{q}_{\alpha}$.

A corollary of this result is that the two functions $g_{2}(\xi)$ and $g_{3}(\xi)$ 
((12) and $(16 a, b))$ can be expressed in terms of the single function $u(\xi)$ or, of course, $U(\xi)$ (19) from which it was derived. In particular, these functions are related as follows:

$$
\begin{aligned}
& g_{2}(\xi)=\frac{(\lambda+2 \mu)\left(\lambda_{u}+\mu\right)}{\left(\lambda_{u}+2 \mu\right)(\lambda+\mu)}\left[1-\frac{\mu\left(\lambda_{u}-\lambda\right)}{(\lambda+2 \mu)\left(\lambda_{u}+\mu\right)}\left(u-\xi u^{\prime}\right)\right] \\
& g_{3}(\xi)=\frac{(\lambda+2 \mu)\left(\lambda_{u}+3 \mu\right)}{\left(\lambda_{u}+2 \mu\right)(\lambda+3 \mu)}\left[1+\frac{\mu\left(\lambda_{u}-\lambda\right)}{(\lambda+2 \mu)\left(\lambda_{u}+3 \mu\right)} u(\xi)\right]
\end{aligned}
$$

The stress field of the fluid dipole has the same structure as the displacement field: it is the difference between the classical elasticity solution based on the undrained moduli for a point force and the point source solution given by (13) and (15). The result is recorded below:

$$
\begin{aligned}
4 \pi r^{3} \sigma_{i j}= & \frac{q_{\alpha}}{\rho_{o} c} \frac{(\lambda+2 \mu)}{\tau}\left\{\left[\frac{\mu}{\lambda u+2 \mu}-\frac{\mu}{\lambda+2 \mu} G_{3}(\xi)\right] x_{\alpha} \delta_{i j}\right. \\
& -\left[\frac{\mu}{\lambda_{u}+2 \mu}-\frac{\mu}{\lambda+2 \mu} G_{2}(\xi)\right]\left(\delta_{i \alpha} x_{j}+\delta_{j \alpha} x_{i}\right) \\
& \left.-3 \frac{x_{i} x_{j} x_{\alpha}}{r^{2}}\left[\frac{\lambda_{u}+\mu}{\lambda_{u}+2 \mu}-\frac{\lambda+\mu}{\lambda+2 \mu} G_{1}(\xi)\right]\right\}
\end{aligned}
$$

This expression corrects that given by Cleary [11] in his equation (43). As noted by Cleary [11] the dipole solution gives no net force on any contour surrounding the origin and the contributions from the two portions of (36) must be equal and opposite. It is apparent from the preceding that the functions $G_{1}, G_{2}$, and $G_{3}$ can also be expressed in terms of $u(\xi)$. 
Concluding Discussion

This note has corrected some expressions given by Cleary [11] for fundamental solutions in linear elastic porous fluid infiltrated solids. More importantly, the corrected expressions reveal the full extent of the correspondence between the point source solutions and fluid mass dipole solutions. In retrospect, this correspondence is an inevitable result of the fact that the governing equations reduce to the usual elasticity equations in the long and short-time limits. An additional result of this analysis is that the time dependence of the point force solution is expressible in terms of a single function which arises in the solution for the displacements due to a continuous fluid mass source.

The rearrangement of the point force solution which has been given here may prove advantageous in the construction of solutions to boundary value problems and simulation of anomalous zones of plasticity, as outlined by cleary [11]. Specifically, some simplification may result from the separation of the solution into two components one of which is a solution to the classical elasticity equations with the undrained moduli and one which is a solution to the homogeneous diffusion equation. However, the difficulty in solving boundary value problems inevitably enters in the coupling introduced in the boundary conditions [3, 7] and it is unlikely that this complication can be avoided.

\section{Acknowledgement}

This work arose in connection with investigations supported by the Earthquake Hazards Reduction Program of the U. S. Geological Survey through Contract No. INT 14-08-0001-19146 to the University of Illinois at Urbana-Champaign. 


\section{$\underline{\text { References }}$}

1. J. McNamee and R. E. Gibson, Displacement functions and linear transforms applied to diffusion through porous elastic media. Quart. J. Mech. App. Math. 13, 99-111 (1960).

2. M. A. Biot, General theory of three dimensional consolidation. J. App. Phys. 12, 155-164 (1941).

3. J. R. Rice and M. P. Cleary, Some basic stress-diffusion solutions for fluid-saturated elastic porous media with compressible constituents. Rev. Geophys. Space Phys. 14, 227-241 (1976).

4. A. Ruina, Influence of coupled deformation-diffusion effects on retardation of hydraulic fracture, in Proc. 19th U. S. Symp. Rock Mech., State line, Nev. edited by Y. S. Kim, pp. 243-246 (1978).

5. J. R. Booker, Time-dependent strain following faulting of a porous medium. J. Geophys. Res. 79, 2037-2044 (1974).

6. J. R. Rice and D. A. Simons, The stabilization of spreading shear faults by coupled deformation-diffusion effects in fluid-infiltrated porous materials. J. Geophys. Res. 81, 5322-5334 (1976).

7. J. R. Rice, J. W. Rudnicki, and D. A. Simons, Deformation of spherical cavities and inclusions in fluid-infiltrated elastic materials. Int. J. Solids Structures 14, 289-303 (1978).

8. J. R. Rice and J. W. Rudnicki, Earthquake precursory effects due to pore fluid stabilization of a weakening fault zone. J. Geophys. Res. 84, 2177-2193 (1979).

9. J. W. Rudnicki, The stabilization of slip on a narrow weakening fault zone by coupled deformation - pore fluid diffusion. Bu11. Seism. Soc. Am. 69, 1011-1026 (1979). 
10. J. R. Rice, Pore fluid processes in the mechanics of earthquake rupture, in Solid Earth Geophysics and Geotechnology edited by S. Nemat-Nasser, Applied Mechanics Division Volume, American Society of Mechanical Engineers, New York, Publication expected November, 1980.

11. M. P. Cleary, Fundamental solutions for a fluid-saturated porous solid, Int. J. Solids Structures 13, 785-808 (1977).

12. H. S. Carslaw and J. C. Jaeger, Conduction of Heat in Solids, 2nd edition, Clarendon Press, Oxford, p. 256 (1959). 


\section{Effects of Fault Interaction on Moment, Stress Drop and Strain Energy Release}

by

J. W. Rudnicki ${ }^{\dagger}$

and

H. Kanamori $i^{*}$

Journal of Geophysical Research, 86, 1785-1793

'Department of Theoretical and Applied Mechanics, University of Illinois, Urbana, IL 61801

* Seismological Laboratory, California Institute of Technology, Pasadena, CA 91125 


\title{
Effects of Fault Interaction on Moment, Stress Drop, and Strain Energy Release
}

\author{
J. W. RUDNICKI \\ Department of Theoretical and Applied Mechanics, University of Illinois, Urbana, Illinois 61801 \\ H. KANAMORI \\ Seismological Laboratory, California Institute of Technology, Pasadena, California 91125
}

\begin{abstract}
Solutions for collinear shear cracks are used to examine quantitatively the effects of fault slip zone interaction on determinations of moment, stress drop, and static energy release. Two models, the barrier model and the asperity model, are considered. In the asperity model, the actual distribution of strengths on a fault plane is idealized as a combination of two limiting cases: areas which slip freely at a uniform value of a residual friction stress and unbroken ligaments or 'asperities' across which slip occurs only at the time of a seismic event. In the barrier model, slip zones separated by unbroken ligaments (barriers) are introduced into a uniformly stressed medium to approximate the nonuniform fault propagation proposed by Das and Aki. The strain energy change due to introducing collinear slip zones or due to breaking the asperities between them is shown to be given by the usual formula for an isolated slip zone with the stress drop replaced by the effective stress. Significant interaction between slip zones occurs only if the length of the asperity is less than half the length of the slip zones. For the case of two collinear slip zones, fracture of the asperity between them is shown to cause a large moment primarily because of the additional displacement which is induced on the adjacent slip zones. For example, if the asperity length is $0.05 l$, where $l$ is the length of each adjacent slip zone, then fracture of the asperity causes a moment almost 1.8 times the moment caused by introducing a slip zone of length $l$. For two collinear slip zones, the local stress drop due to fracture of the separating asperity is shown to become unbounded as the asperity length goes to zero, but in the same linit the stress drop averaged over the entire fault length is approximately equal to the apparent stress drop inferred for an isolated fault of the same moment and total fault length. This apparent stress drop is approximately equal (within a factor of 2 or 3 ) to the effective stress and hence can be used in the usual formula to give a good estimate of the strain energy change. For the barrier model, numerical results are given for the ratio of the stress drop calculated on the assumption of an isolated slip zone to the true stress drop. For example, in the case of two collinear slip zones of length $l$ separated by a barrier of length $0.2 l$, this ratio is 0.5 , whereas for a barrier length equal to that of the adjacent slip zones, the ratio is 0.24 . Stress drop estimates become worse with increasing number of fault segments.
\end{abstract}

\section{INTRODUCTION}

Although earthquake faulting is often idealized as smoothly varying slip on an isolated zone, it has become increasingly evident from detailed analysis of seismograms that slip on earthquake faults is very irregular. This irregularity is particularly evident in large earthquakes which have been shown to consist of a number of distinct events [e.g., Imamura, 1937; Miyamura et al., 1964; Wyss and Brune, 1967; Trifunac and Brune, 1970; Nagamune, 1971]. More recent studies on the waveforms of these multiple shocks have unravelled some of the details of the stress release in these complex events [e.g., Kanamori and Stewart, 1978; Rial, 1978]. Also, the occurrence and distribution of foreshocks [Jones and Molnar, 1979; Ishida and Kanamori, 1978, 1980] and doublet earthquakes [Lay and Kanamori, 1980] have been used to infer patterns of fault plane heterogeneity. Thus it seems likely that the distribution of slip and stress on fault planes is very heterogeneous. For faults that have previously undergone large amounts of slip, the stress on much of the fault plane may be equal to a residual value of the friction stress. However, there are also likely to be regions having higher resistance to slip. These regions are usually termed 'asperities' (this term is not meant to be interpreted in the specific sense in which it is used in the physics of friction of referring to a geometric surface roughness [e.g.,

Copyright (C) 1981 by the Anterican Geophysical Union.
Bowden and Tabor, 1973]), and they may be due to either material or geometric effects.

In the interpretation of these multiple shocks, it is often assumed, implicitly or explicitly, that the individual events in the multiple-shock sequence represent failure of such asperities. In the following discussion we refer to this type of faulting as the asperity model. Furthermore, it has been suggested [Kanamori, 1978; Das and Aki, 1977b; Aki, 1979; Mikumo and Miyatake, 1979; Ishida and Kanamori, 1978, 1980; Lay and Kanamori, 1980] that asperities may control the pattern of earthquake occurrence, for example, foreshock-mainshock, swarm, doublet, etc.: as tectonic stress is increased, weaker asperities fail and the resulting slip alters the stress in the remaining stronger asperities.

On the other hand, Das and $A k i[1977 b]$ have suggested, on the basis of numerical experiments in dynamic crack propagation, that a propagating fault may leave behind unbroken barriers, that is, high-strength areas of the fault plane. They called this type of faulting the barrier model. Several authors [Madariaga, 1979; Rice, 1979b; Aki, 1979] have shown that seismic parameters inferred by assuming uniform slip or stress drop can be in considerable error if the earthquake is represented by the barrier model.

Although these two models would represent the two extreme cases of the actual earthquake process, they are useful for characterizing fault plane heterogeneity.

Madariaga [1979] has given an elegant formula relating 


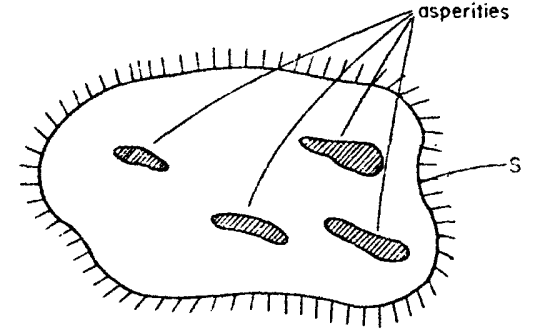

Fig. 1. Schematic idealization of a fault plane. Outside the curve labeled $\mathbf{S}$ there is no relative displacement of the fault surfaces. Inside curve $S$ the fault surfaces slip freely at a residual friction stress except for the cross-hatched areas which represent 'asperities' across which there is relative displacement only during seismic events.

stress drop and moment under general circumstances, and he uses this to give some approximate results for a few special cases. It is, however, a matter of practical interest to determine the magnitude of effects of fault zone interaction on moment, static stress drop, and strain energy change. This paper examines quantitatively these effects primarily by using the asperity model.

We idealize the distribution of strengths on the fault plane as a combination of the following two limiting cases: locked segments which slip only at the time of a seismic event and segments which freely slip at a residual friction stress. As a more specific illustration, consider the idealized fault plane shown in Figure 1. The region outside the curve labeled $S$ is idealized as locked. Inside curve $S$, the cross-hatched areas are also locked and represent asperities or portions of the fault which require a greater level of applied stress in or ter for slip to occur. The remainder of the fault inside curve $S$ sustains a uniform friction stress $\tau_{f}$ but slips freely at stresses larger than $\tau_{f}$. This model can be further idealized as a distribution of plane (or antiplane) strain (i.e., of infinite extent in one direction) shear cracks as shown in Figure 2. The variations along the fault plane of stress and of relative slip are shown schematically. The cracks model slip zones which sustain the uniform friction stress $\tau_{f}$, and the ligaments between the cracks (slip zones) represent asperities.

Although this model is oversimplified, it has the advantage that existing results for collinear cracks can be used to obtain exact, closed-form expressions which make possible a quantitative assessment of the effects due to the interaction of slip zones. Because the idealization is a limiting one, the results given here can reasonably be expected to yield bounds for more realistic situations. Moreover, the results provide some specific numerical examples of the general relations between moment and stress drop derived by Madariaga [1979] and illustrate the extent to which small strong asperities or barriers can control the pattern of stress release. Although many of the general results illustrated here are well known, for example, that stress drop in a small asperity will exceed the average stress drop, the numerical results permit a quantitative assessment of the magnitude of these effects and the extent to which they depend on the separation distances of slip zones and asperities or barriers.

The next section discusses some preliminary considerations and reviews some results for isolated slip zones before considering the interaction of slip zones separated by asperities.

\section{SOME RESULTS FOR ISOLATED SLIP ZONES}

Consider an isolated slip zone of length $l$ which is embedded in an infinite linear elastic body (Figure 3 ). For conve- nience, the slip zone is considered to be of infinite extent in one direction so that plane strain conditions apply. The slip zone sustains a uniform frictional stress $\tau_{\text {, }}$ and the body is loaded in the far field by the shear stress $\tau_{\infty}$. The stresses near the ends of such a slip zone are proportional to $r^{-1 / 2}$, where $r$ is the distance from the edge of the zone, and are characterized by the stress intensity factor K [e.g., Knott, 1973; Rice, 1968], which is defined by the following relation:

$$
K=\lim _{r \rightarrow 0}(2 \pi r)^{1 / 2} \sigma_{x y}
$$

where $\sigma_{x y}$ is the shear stress on the plane ahead of the slip zone. If the slip zone shown in Figure 3 encloses no net dislocation (so that the displacement field outside the zone is single valued), the stress intensity factor is

$$
K_{i}=(\pi l / 2)^{1 / 2}\left(\tau_{\infty}-\tau_{j}\right)
$$

where the subscript denotes the value for an isolated slip zone.

Of course, in any real material, the stresses at the edge of the slip zone will not be singular but instead will be alleviated by processes of inelastic deformation. If, however, the characteristic dimension of this zone of inelasticity or 'breakdown' zone is small by comparison with other length scales in the problem (e.g., length of slip zone, distance to boundaries, etc.), then the intensity of deformation in this zone is characterized by $\boldsymbol{K}$. More precisely, the boundary conditions on the breakdown zone are fixed by the singular stress of the surrounding linear elastic field. In the absence of any detailed information about processes in the breakdown zone, the neartip stress field can be idealized as singular with the understanding that $K$ characterizes the actual near-tip field (Rice [1968]; also see $R$ Rudnicki [1980] for a recent discussion).

Note that a uniform stress $-\tau_{\infty}$ or $-\tau_{\text {, }}$ may be superposed on the stress field in Figure 3 without affecting the relative displacement of the fault surfaces. Hence the relative displacement may be regarded as due to the application of stresses $\tau_{\infty}-\tau_{f}$ to the fault surfaces with zero stress at infinity or to application of stresses $\tau_{\infty}-\tau_{f}$ at infinity with a frictionless fault surface. In any case the relative displacement is

$$
\delta_{i}(x)=2(1-\nu) \tau \mu^{-1}\left[(l / 2)^{2}-x^{2}\right]^{1 / 2}
$$

where $\tau=\tau_{\infty}-\tau_{f}$ is the effective stress (which is equal in this case to the stress drop), $\mu$ is the shear modulus, $\nu$ is Poisson's ratio, and the subscript again denotes the value for an isolated slip zone. The average relative slip is

$$
\delta_{i}(l)=\pi(1-\nu) \tau \mu^{-1} l / 4
$$

The static moment (per unit thickness) is

$$
M=\int_{-1 / 2}^{1 / 2} \mu \delta(x) d x
$$

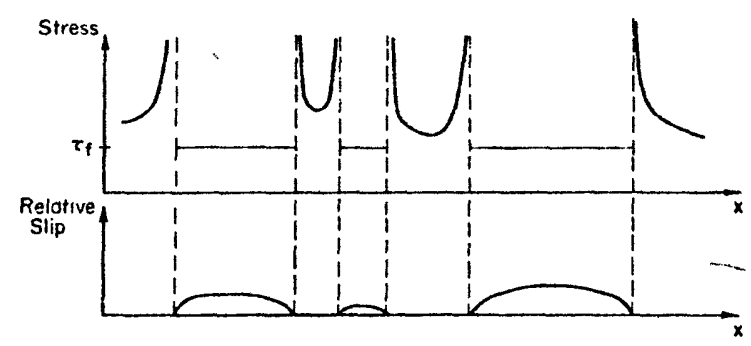

Fig. 2. Plane strain idealization of a fault plane as a combination of areas which freely slip at the friction stress $\tau_{f}$ and areas of asperities which undergo no relative displacement. 
where the integration limits imply that the coordinate origin is at the crack center, or, for constant $\mu$,

$$
M=\mu l \delta
$$

Substituting from (4) yields

$$
M_{i}(l)=(1-\nu) \tau \pi(l / 2)^{2}
$$

for the isolated slip zone. In this formula it is, of course, the seismic moment which is determined most accurately from observations. The stress drop $\tau$ is typically inferred from (7) or a similar formula on the basis of the observed moment and the observed or estimated fault length. As has been discussed in detail by Madariaga [1977], this stress drop is not generally equal to the average of the true stress drop. However, it will be shown that for the geometry considered here the difference between these quantities is not great.

\section{Strain Energy Change}

Although the strain energy change during faulting cannot, in general, be determined (because the friction on the fault is unknown), the strain energy change does provide an upper bound to the energy available for seismic wave radiation. The strain energy change caused by introducing the slip zone can be computed as the negative of the work done by the fault surface tractions in restoring the body to the unslipped state [Rice, 1966]. If the stress on the fault is reduced to the residual friction stress $\tau_{f}$, this work is

$$
W=\tau_{f} \delta l+\frac{1}{2} \int_{-l / 2}^{1 / 2} \Delta \tau(x) \delta(x) d x
$$

where $\Delta \tau(x)$ is the stress drop. For the isolated zone, $\Delta \tau(x)=$ $\tau_{\infty}-\tau_{f}$ and, consequently,

$$
W=\tau, l \delta+\frac{1}{2}\left(\tau_{\infty}-\tau_{f}\right) l \delta
$$

The usual interpretation of this equation is that the first term is equal to the heat generated by faulting [e.g., Orowan, 1960] and the second term is available for seismic energy radiation. Comparison of (6) with (9) reveals that this second term, which Kanamori [1977] calls $W_{0}$, can be expressed in terms of the moment as

$$
W_{0}=\tau M / 2 \mu
$$

where $\tau=\tau_{\infty}-\tau_{f}$. Of course, the actual seismic energy radiated during faulting depends on the deatils of the rupture process (whereas the strain energy change does not; this will be demonstrated below for the specific model considered here) and cannot be determined from comparison of the static

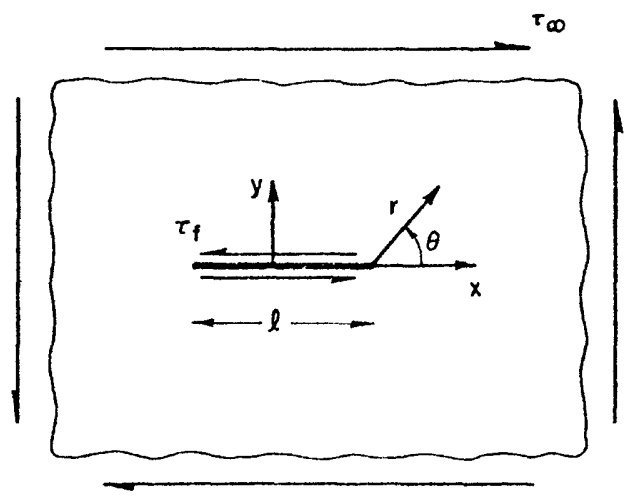

Fig. 3. An isolated slip zone. The resistive frictional stress is $\tau_{f}$, and the slip zone is loaded in the far field by $\tau_{\text {. }}$.

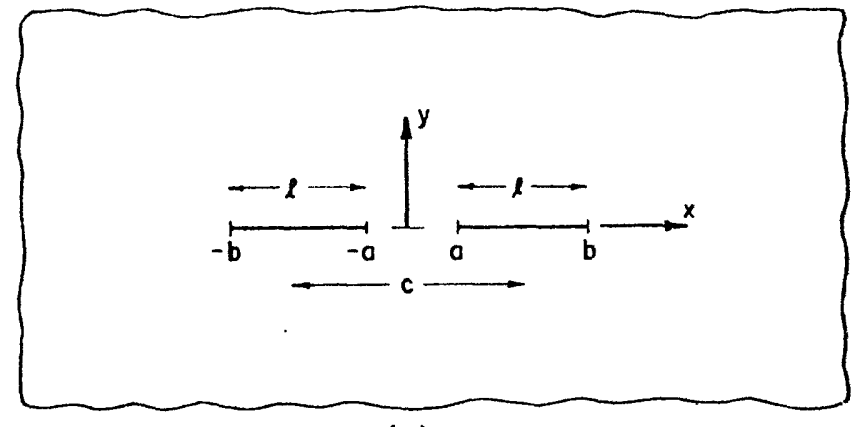

(a)

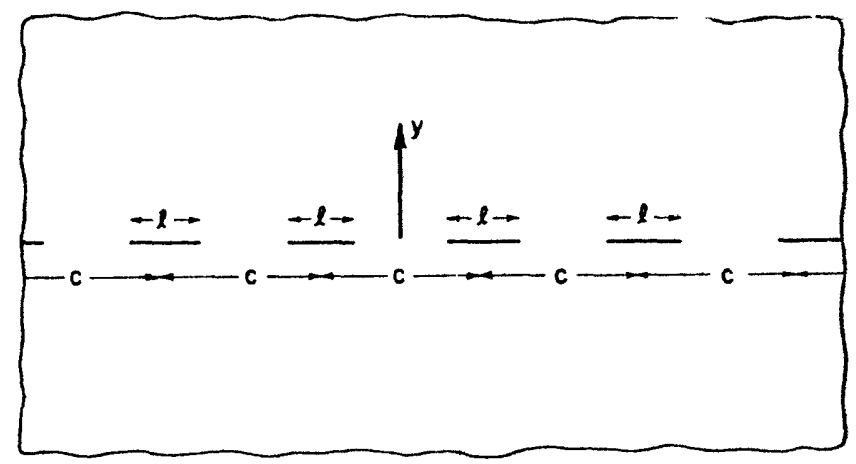

(b)

Fig. 4. (a) Geometry for two collinear slip zones. (b) Geometry for an infinite periodic array of collinear slip zones.

end-states [Kostrov, 1974]. However, Kanamori [1977] has shown that for large earthquakes the energy computed from (10) agrees well with the energy determined by using the observed magnitude and the Gutenberg-Richter relation (See Richter [1958] with correction noted by Kanamori and Anderson [1975].) In the following, we show that the strain energy change is also given by (9) for two specific types of nonuniform faulting.

First, we consider an initially unbroken fault. Then failure occurs on an array of collinear slip zones separated by ligaments as shown schematically in Figure 2. This case corresponds to the barrier model. The strain energy change is still given by (8) if the total length of the fault zone is $l$. Although the slip is very nonuniform, wherever the relative slip is nonzero the stress drop is equal to the effective stress $\left(\tau_{\infty}-\tau_{f}\right)$, and consequently (8) again reduces to (9).

In the second case we suppose that the fault plane is already segmented in the initial state and failure of the asperities causes reduction of the stress to $\tau_{f}$ everywhere on a zone of length $l$. This case corresponds to the asperity model. Again the pattern of stress drop is very complex: it is zero on portions of the fault plane which already sustained the residual friction stress $\tau_{f}$, but it is nonzero and spatially varying where asperities existed. Nevertheless, the strain energy change is again given by (8). Note, however, that wherever the stress drop is nonzero (at positions which were occupied by asperities), the relative slip is exactly equal to the relative displacement for an isolated zone of length $l$. Hence $\delta(x)$ in the second term of $(8)$ may, in this case, be replaced by $\delta_{i}(x)$ from (3). The reciprocal theorem of linear elasticity (e.g., Love [1927]; also see Madariaga [1979] for a more detailed treatment of a similar application of the reciprocal theorem) requires that

$$
\int_{-1 / 2}^{1 / 2}(\Delta \tau), \delta(x) d x=\int_{-1 / 2}^{1 / 2} \Delta \tau(x) \delta_{1}(x) d x
$$




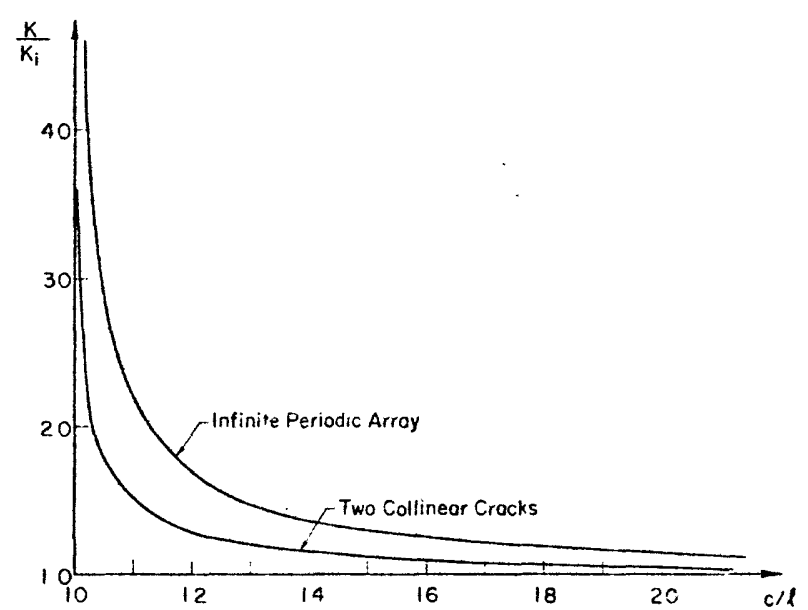

Fig. 5. Plot of stress intensity factor for fault tip at $x= \pm a$ in Figure $4 a$ (equation (11)) and for Figure $4 b$ (equation (12)) as a function of $c / l$.

where the subscript $i$ in the first integral denotes the stress drop for an isolated slip zone. The stress drop for an isolated zone is, however, uniform and equal to the effective stress so that the strain energy change can again be expressed as in (9).

These examples demonstrate that for a fault zone of a given length, which may comprise any rumber of collinear slip zones, the strain energy change is fully deterruined by the effective stress and the average displacement regardless of the detailed distribution of constituent slip zones or of stress drop. Although this result is fully expected from the theory of linear elasticity, it often seems to be overlooked in seismology. The apparent reason for this is that the actual stress drop, which appears in the second term of (8), is not known but must be inferred from formulae like (7). Hence calculations based on (10) reflect possible inaccuracies in determining the stress drop by assuming an isolated slip zone with uniform stress drop.

Before proceeding to specific calculations of moment and stress drop for collinear slip zones, we present some results for the interaction of slip zone stress fields.

\section{INTERACTION OF SLIP ZONE STRESS FIELDS}

Although the solution for an arbitrary configuration of collinear cracks can formally be established using the powerful complex variable methods of Muskhelvishvili [1953], only the limiting cases of two collinear cracks [Willmore, 1949; Tranter, 1961; Barenblatt, 1962; Erdogan, 1962, Lowengrub and Srivastava; 1968] and an infinite periodic array of collinear cracks [Irwin, 1957; Koiter, 1959] need be considered here. The solutions for these cases (and for many others) have been summarized by Tada et al. [1973]. For two collinear cracks, the geometry is shown in Figure $4 a$. The stress intensity factor at the interior fault tip $(x= \pm a)$ is given by

$$
K_{2}=\tau(\pi a)^{1 / 2} \frac{b^{2} E(m) / F(m)-a^{2}}{a\left(b^{2}-a^{2}\right)^{1 / 2}}
$$

where $\tau=\tau_{\infty}-\tau_{f}$ is the excess of the far field stress over the frictional stress, $m^{2}=1-a^{2} / b^{2}$, and $F(m)$ and $E(m)$ are complete elliptic integrals of the first and second kinds, respectively:

$$
\begin{aligned}
& F(m)=\int_{0}^{\pi / 2}\left(1-m^{2} \sin ^{2} \theta\right)^{-1 / 2} d \theta \\
& E(m)=\int_{0}^{\pi / 2}\left(1-m^{2} \sin ^{2} \theta\right)^{1 / 2} d \theta
\end{aligned}
$$

The center-to-center distance between the slip zones is $c=a$ $+b$ and the length of each zone is $l=b-a$. The geometry for the infinite periodic array of slip zones is shown in Figure $4 b$, and the stress intensity factor is

$$
K_{\infty}=\tau[c \tan (\pi l / 2 c)]^{1 / 2}
$$

The interaction between the stress fields can be evaluated by examination of Figure 5 , which plots $K_{2}$ and $K_{\infty}$ divided by $K_{i}$, the stress intensity factor for an isolated crack, as 2 function of $c / l$. Figure 5 makes it clear that interaction is significant only when the slipping zones are very close together. This result is not unexpected, since the stress field near the tip of the slip zone does die off rapidly (as $r^{-1 / 2}$ ) with distance. The elevation of the stress intensity factor over the value of the isolated slip zone is, of course, greatest for the infinite array of slip zones. Even for this configuration, however, the stress intensity factor is greater than $K_{i}$ by only slightly more than $10 \%$ when the zones are separated by a distance equal to their length $(c / l=2)$. Hence for greater separations, say, $c / l>$ 3 , the slip zones are effectively isolated. Although the interaction may be amplified by three-dimensional effects, the results here suggest that interaction is significant only if the asperities or locked portions occupy a small fraction of the fault plane.

A lower limit to the ratio of asperity length to slip zone length can be estimated by requiring that the average stress in the asperity which is available for release in a seismic event does not exceed the ultimate (peak) strength of brittle rock. ( $A k i$ [1979] has made similar estimates within the context of the barrier model.) For an asperity which has a length small by comparison to the lengths of adjacent slip zones, the stress in the asperity can be approximated by the singular term in the crack-tip expansion, that is, $K(2 \pi r)^{-1 / 2}$, where $K$ is the ap-

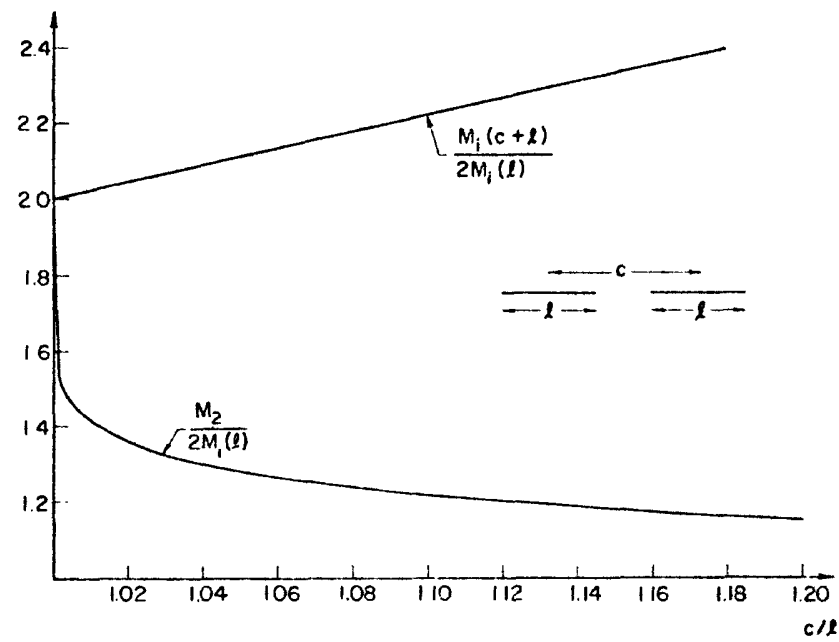

Fig. 6. Moment due to the introduction of two collinear slip zones of length $/$ divided by 2 times the moment due to introduction of an isolated slip zone of length $/$ plotted as a function of $c / l$ (see (16) and (7)). Also shown is the moment for an isolated zone of length $l$ divided by that for an isolated zone of length $c+l$. 
propriate stress intensity factor and $r$ is the distance from the edge of the slip zone. Thus the average stress in an asperity of length $c-l$ is

$$
\bar{\sigma}=\tau\left(K / K_{i}\right)[c / l-1]^{-1 / 2}
$$

where the numerator and denominator have been multiplied by $K_{t}$, the stress intensity factor for an isolated slip zone, and $\tau$ $=\tau_{\infty}-\tau_{f}$ is the effective stress. (Equation (13) actually gives an estimate of the stress in excess of the residual friction value.) Rearranging and setting $\sigma=\sigma_{u l t}$, the ultimate stress, yield

$$
\frac{c}{l}=1+\left(\frac{K}{K_{i}} \frac{\tau}{\sigma_{u l l}}\right)^{2}
$$

although, of course $K / K$ itself depends on $c / l$ as shown in Figure 5. On the basis of laboratory experiments [e.g., Jaeger and Cook, 1976], a representative value for $\sigma_{u l t}$ might be $10^{3}$ bars $\left(=10^{2} \mathrm{MPa}\right)$, and $\tau$ is presumably in the range of typical stress drops, say, $10-100$ bars (1-10 MPa). For $\tau / \sigma_{\text {ull }}$ equal to $10^{-2}$ and $10^{-1}$, (14) yields values of $c / l$ equal to 1.006 and 1.068 , respectively, for the infinite periodic array. The corresponding values of $c / l$ for two collinear cracks are 1.003 and 1.038. Hence $c / l=1.006$ seems to be a reasonable lower limit.

Of course, the above estimates assume that the asperity remains essentially elastic and because of the proximity of the slip zones needed to cause significant interaction, it is worthwhile reexamining the adequacy of this assumption. As mentioned earlier, the formulation which regards the fault-tip stress field as singular is equivalent to one which takes explicit account of departures from linear elasticity in a breakdown zone as long as the characteristic length of this zone is small by comparison with other lengths in the problems. Rice [1979a] has estimated breakdown zone sizes from $11 \mathrm{~mm}$ to 1 $\mathrm{m}$ based on laboratory experiments of sliding friction. If these results are representative, it seems likely that the asperity size will be much greater than the end zone size. For a slip zone length of $1 \mathrm{~km}$ and the lower limit for $c / l=1.006$, the asperity length is $6 \mathrm{~m}$. Rudnicki [1979], however, has suggested, based on model experiments of Barton [1972, 1973], that larger end zone sizes, perhaps of the order of a hundred meters, may occur in situ, and it is conceivable that asperities may be of this size. Unfortunately, there seem to be no accurate estimates of sizes of either asperities or breakdown zones in the field, and Rudnicki [1980] has discussed the difficulties of extrapolating friction experiments to the field. In view of these undertainties, the approximation of a singular stress field seems adequate. In any case, the stress field which is given by the resulting values for the stress intensity factors can be expected to describe adequately the average stress level in the asperity.

\section{MOMENT AND STRESS DROP}

Since the results are very different for the two models, the barrier and the asperity models, we discuss them separately. In the barrier model we compare the calculated moment and stress drop for the introduction of a segmented fault into a uniform state of stress with the corresponding quantities for an isolated fault or slip zone. In the asperity model the moment and stress drop due to failure of an asperity between existing slip zones are calculated. (Here 'failure' means that the stress is reduced to the residual friction value $\tau_{f}$.) Again the results are compared with those for isolated slip zones. As

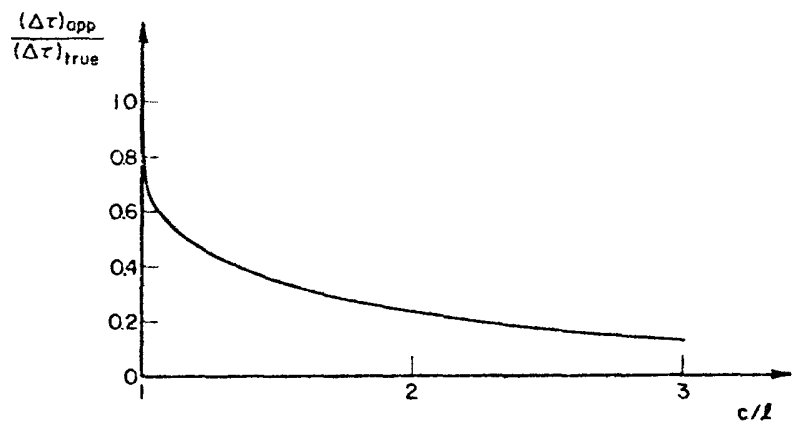

Fig. 7. Apparent stress drop (based on the assumption of an isolated slip zone) divided by actual stress drop for a fault having two segments of length $l$ separated by the center-to-center distance $c$.

demonstrated earlier, the strain energy change and hence $W_{0}$ is completely determined, for a given fault length and effective stress, by the average displacement and consequently by the moment from (10). Thus despite the evident importance of $W_{0}$ as a seismological parameter, the results here have been expressed in terms of moment and stress drop because the moment can be determined more directly from observations and because errors in the estimation of $W_{0}$ (say, from (10)) result from errors in the stress drop.

\section{Barrier Model}

The moment due to introducing two widely separated slip zones of length $l$ is simply $2 M_{i}(l)$, where $M_{i}$ is given by (7). As the two slip zones become closer together, the moment increases and the limiting value occurs when the slip zones are adjacent so that they approach a single zone of length $2 l$. In this limit the moment is $M_{i}(2 l)=4 M_{i}(l)$. For intermediate cases it is necessary to evaluate the moment by using (5). For the two collinear slip zones shown in Figure $4 a$, the relative slip of the fault surfaces can be determined from expressions given by Erdogan [1962] and, as shown in the appendix, the average slip is

$$
\delta_{2}=\pi(1-v) \tau \mu^{-1} b \frac{\left[1+(a / b)^{2}-2 \lambda^{2}\right]}{2(1-a / b)}
$$

where $\lambda^{2}=E(m) / F(m)$ and $m^{2}=1-a^{2} / b^{2}$. Hence from (5) the moment is

$$
M_{2}=2 \pi(1-\nu) \tau(b / 2)^{2}\left[1+(a / b)^{2}-2 \lambda^{2}\right]
$$

$M_{2}$ divided by twice the moment for an isolated zone of length $l=b-a$ is plotted in Figure 6 as a function of $c / l$ (where $c=$ $a+b$ ). As shown in Figure $6, M_{2}=4 M_{i}$ when $c / l=1$ (i.e., $a / b$ $=0$ ) and $M_{2}$ approaches $2 M_{i}$ as $c / l$ approaches infinity (i.e., $a / b=1)$. Also plotted is $M_{i}(c+l) / 2 M_{i}(l)$. Figure 6 demonstrates that the effect of interaction is very slight unless the ratio of asperity size to slip zone length is extremely small. For example, for $c / l=1.2$ (ratio of asperity length to slip zone length is 0.2 ), the moment $M_{2}$ differs by only $15 \%$ from the combined moments of two isolated zones of length $l$; for $c / l=$ 2.0 , the difference is only about $3 \%$.

In calculating the moment it was assumed that the stress drop for each fault segment was $\tau_{\infty}-\tau_{\text {, }}$. However, as noted earlier, the moment is the quantity which can be determined accurately by observations, whereas the stress drop is typically inferred from formulae like (7) which assume that the fault is a single isolated zone. Thus it is perhaps more relevant for observations to determine for a fixed moment and fault length 


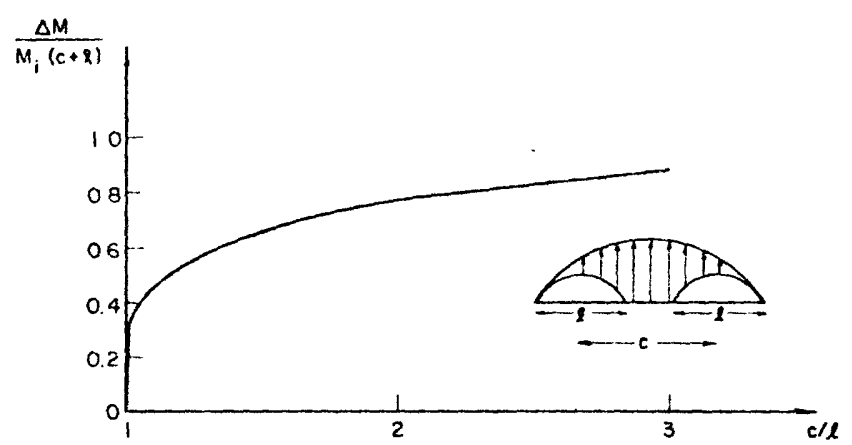

Fig. 8. Moment due to failure of asperity between two slip zones divided by the moment due to uniform stress drop on an isolated slip zone of length $c+l$.

the ratio between the actual stress drop and the apparent stress drop inferred from (7). Equating the moments calculated from (6) by using (15) with $\tau=\Delta \tau_{\text {true }}$ and from (7) with l $=2 b$ and $\tau=\Delta \tau_{a p p}$ yields

$$
\frac{\Delta \tau_{\text {uruc }}}{\Delta \tau_{\text {app }}}=\frac{1}{1+(a / b)^{2}-2 \lambda^{2}}
$$

The reciprocal of this expression is plotted versus $c / l[=(1+$ $a / b) /(1-a / b)]$ in Figure 7 . For very small asperity lengths the two stress drops are approximately equal, but more generally the apparent stress drop can significantly underestimate the true stress drop. For example, if $c / l=1.2$, the true stress drop is roughly twice the apparent value, but if the asperity length is equal to the length of the adjacent slip zones $(c / l=$ 2 ), the apparent stress drop is only $24 \%$ of the true stress drop.

Rice $[1979 b]$ has used results for the infinite periodic array of collinear cracks to obtain a formula corresponding to (17) for a large number of segments. Rice's expression is (in our notation)

$$
\frac{\Delta \tau_{\text {true }}}{\Delta \tau_{\text {app }}}=\frac{n \pi^{2}}{8 \ln \left|\sec \frac{\pi}{2}(1-l / c)\right|}
$$

where $n \gg 1$ is the number of slip zone segments. For $c / l=$ $1.01,1.1,1.2$, and 1.5 this ratio is $0.30 n, 0.63 n, 0.91 n$, and $1.78 n$, respectively. (The numerical values given by $R$ ice [1979b] for $(1-l / c)=0.25,0.1$, and 0.01 are in error and should be replaced by $1.28 n, 0.67 n$, and $0.30 n$, respectively.) Comparison of (18) with (17) reveals that the difference between the actual and apparent stress drops is greater for the larger numbers of slip zone segments. For $c / l=1.2, \Delta \tau_{\text {true }}=2$ $\Delta \tau_{\text {app }}$ for two segments, but for a large number of segments, say, 20-25, the actual stress drop in each segment is 20-25 times the apparent value.

The calculations in this section give specific numerical examples of the general result obtained by Madariaga [1979]: for a given moment and fault length the segmented fault represented by the barrier model has high stress drop than the isolated fault.

\section{Asperity Model}

In this section we demonstrate that the failure of an asperity, which may occupy a very small portion of the fault plane, can cause a relatively large moment because of the additional displacement induced on the adjacent slip zones. Because the body has been assumed to be linearly elastic, this moment is simply equal to the difference

$$
\Delta M=M_{1}(c+l)-M_{2}
$$

where $M_{2}$ is given by (16) and $M_{1}(l)$ is given by (7). This difference divided by $2 M_{\Lambda}(l)$ is the difference between the two curves in Figure 6, and in Figure $8, \Delta M / M_{i}(c+l)$ is plotted as a function of $c / l$. (For fixed stress drop, $M_{2} / M_{i}(c+l)$ is equal to the reciprocal of the right-hand side of (17) so that Figure 7 is also a plot of $1-\Delta M / M_{N}(c+l)$.)

For values of $c / l$ greater than about 2 , the ratio $\Delta M / M_{i}(c+$ $l)$ is roughly unity, and the moment $\Delta M$ differs from $M_{i}(c+l)$ by less than $20 \%$. Thus if the asperity length is greater than about one third of the total length which slips during failure of the asperity, the effect of the preexisting slip zones on the moment is relatively small (Figure 8). Note, however, that the moment due to failure of the asperity can be relatively large even if the asperity is very small. For example, when the asperity length is only $5 \%$ of the preexisting slip zone length (for $c /$ $l=1.05$, the asperity length is $2 \%$ of the total length which slips during failure of the asperity), the moment due to failure of the asperity is 1.8 times the moment due to creating an isolated slip zone of length $l$ or about $40 \%$ of the moment due to breaking the entire slip zone of length $c+l$. As mentioned earlier, this relatively large moment is due to the additional displacement induced on the already existing slip zones. Because the strength of these zones has already been reduced to the residual friction level $\tau_{f}$, these portions of the fault plane undergo zero stress drop during the failure of the asperity. The effect of this on the average stress drop will be examined next.

If the slip zones are widely separated, the stress on the asperity will be roughly equal to the value of the stress applied in the far field $\left(\tau_{\infty}\right)$ except very near the edges of the slip zones. Consequently, failure of the asperity will be accompanied by a stress drop approximately equal to $\tau_{\infty}-\tau_{f}$. If, however, the slip zones are closer together, the average stress on the asperity will exceed $\tau_{\infty}$ (as demonstrated by Figure 5), and the corresponding stress drop due to failure of the asperity is increased. Of course, the case of most interest is when the asperity between the slip zones is small by comparison to the slip zone length because this is the case for which the presence of the neighboring slip zones is likely to induce failure of the asperity. Specifically, the calculations will demonstrate that the stress drop averaged over the area of a small asperity can be very large, whereas the average over the entire zone which slips (during failure of the asperity) is small.

For the two collinear slip zones shown in Figure $4 a$, the stress on the plane between the slip zones can be determined from expressions given by Erdogan [1962]. The result is

$$
\tau_{u s p}=\left(\tau_{\propto}-\tau_{f}\right) \frac{\left(b^{2} \lambda^{2}-x^{2}\right)}{\left[\left(a^{2}-x^{2}\right)\left(b^{2}-x^{2}\right)\right]^{1 / 2}}+\tau_{\zeta}
$$

where $-a<x<a, \tau_{\infty}$ is the stress applied in the far field, $\tau_{f}$ is the residual friction stress, and $\lambda^{2}=E(m) / F(m)$. Because the stress in the asperity is assumed to be reduced to $\tau_{f}$ by failure. the stress drop is simply given by the first term of (20). Consequently, the average stress drop in the asperity is simply

$$
\Delta \tilde{\tau}_{a, p}=\frac{\tau}{a} \int_{0}^{a} \frac{\left(b^{2} \lambda^{2}-x^{2}\right) d x}{\left[\left(a^{2}-x^{2}\right)\left(b^{2}-x^{2}\right)\right]^{1 / 2}}
$$


where $\tau=\tau_{\infty}-\tau_{f}$. This integral can be expressed as [Abramowitz and Stegun, 1964, p. 596, equation 17.3.13]

$$
\Delta \bar{\tau}_{\text {asp }}=\tau(b / a)[(2 / \pi) F(m)]^{-1}
$$

Because the stress on other portions of the slip zones has already been reduced to $\tau_{f}$, the stress drop here is zero (although there is, of course, additional slip caused by failure of the asperity). Consequently, the average stress drop for the entire slip zone is obtained by multiplying (22) by $a / b$ or

$$
\Delta \bar{\tau}_{f t}=\tau[(2 / \pi) F(m)]^{-1}
$$

This relation between the average stress drop in the asperity and the average stress drop of the slip zone is a special case of the more general relation established by Madariaga. (See equation (15) of Madariaga [1979].) It is straightforward to demonstrate from the properties of $F(m)$ [e.g., Abramowitz and Stegun, 1964, equation 17.3.26] that $\Delta \bar{\tau}_{\text {asp }}$ approaches infinity as $a / b$ approaches zero (or $c / l=1$ ), but that $\Delta \tilde{\tau}_{f t}=0$ in this same limit. Thus although the local stress drop in the asperity can be large, it occurs over such a small area that its contribution to the average stress drop of the entire fault is very small. $\Delta \bar{\tau}_{f t u} / \tau$ and $\left(\Delta \bar{\tau}_{\text {asp }} / \tau\right)^{-1}$ are plotted in Figure 9.

The stress drop defined by (20) is the true stress drop, that is, the stress on the fault plane prior to slip minus that after slip. As noted earlier, the true stress drop is generaily not known; rather an apparent stress drop is inferred from (7). Thus, letting $\tau=\Delta \tau_{a p p}$ and $l=2 b$ in the right-hand side of (7) and setting the left-hand side equal to $\Delta M$ yield

$$
\Delta \tau_{a p p}=\tau\left[2 \lambda^{2}-(a / b)^{2}\right]
$$

The ratio of the apparent stress drop to the true average stress drop is

$$
\frac{\Delta \tau_{\text {app }}}{\Delta \bar{\tau}_{f t t}}=\frac{2}{\pi}\left[2 E(m)-\left(1-m^{2}\right) F(m)\right]
$$

where $m^{2}=1-a^{2} / b^{2}$. This ratio varies monotonically from unity when $c / l=1$ (i.e., $a / b=0)$ to $4 / \pi$ when $c / l=\infty(a / b=$ 1). Hence the apparent stress drop calculated from (7) is a good estimate for the true average stress drop.

Although the apparent stress drop does not differ greatly from the true average stress drop, both quantities underestimate the effective stress for small values of $c / l$. For $c / l=$ 1.01, $\Delta \tau_{\text {app }}$ underestimates the effective stress by a factor of more than 3 and $\Delta \bar{\tau}_{f t}$ by a factor of more than 5 . Hence if the stress drop is assumed equal to the effective stress (as is the case for an isolated zone) for the purpose of computing $W_{0}$ from (10), this quantity may be underestimatcd by a significant amount. If, however, $c / l>1.2, \Delta \tau_{a p p}$ agrees with the effective stress to a factor of 2 , and the difference is probably not significant.

\section{CONCluding Discussion}

Although our model is too simple to describe a real fault in detail, it does make it possible to evaluate quantitatively the effects of the interaction of slip zones separated by asperities. The results are not surprising, but they do demonstrate the dramatic effects that small strong asperities can have on fault processes. In particular, the failure of a small strong asperity can cause a relatively large moment. This result is primarily due to the additional displacement, which is induced by failure of the asperity, on the adjacent already weakened slip

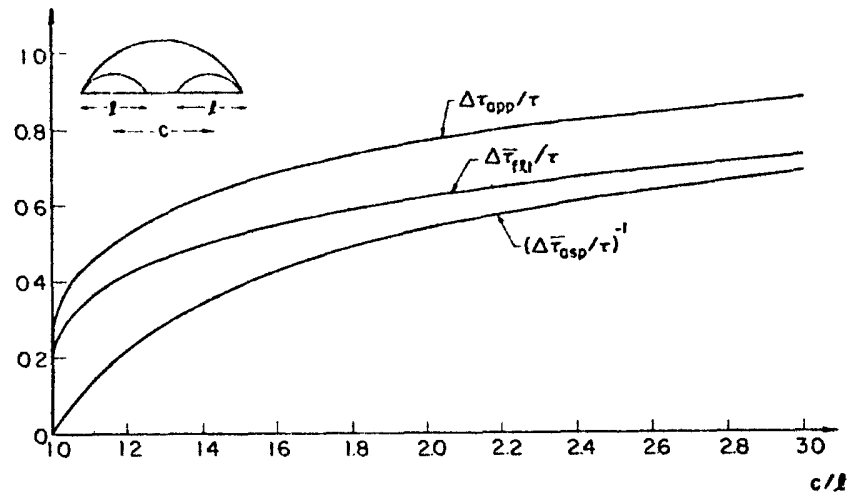

Fig. 9. Average stress drop due to failure of the asperity between two collinear slip zones divided by effective stress as a function of $c / l$. $\Delta \bar{\tau}_{\text {asp }}$ is the stress drop averaged over the width of the asperity (note that the reciprocal of $\delta \bar{\tau}_{a s p} / \tau$ is plotted) and $\Delta \bar{\tau}_{f l}$ is the stress drop averaged over the entire region which slips (see (22) and (23)). $\Delta \bar{\tau}_{\text {app }}$ is the apparent stress drop inferred by assuming uniform stress drop on an isolated fault of length $c+l$.

zones. Consequently, slip on these zones might be said to 'prepare' the fault for large relative displacements due to failure of the strong asperity. The failure of such an asperity can be associated with a large local stress drop, but the stress drop when averaged over the entire slipping region is approximately equal to the stress drop obtained by assuming slip occurred on an isolated fault of the same moment and length. This result suggests itself as a possible explanation for why the observed stress drops for large earthquakes (which are usually an average over the entire slipping region) are relatively constant despite possible heterogeneity of the fault zone [e.g., Kanamori and Anderson, 1975]. Moreover, unless the ratio of asperity size to fault length is very small, this stress drop is a good estimate (within a factor of 2 ) of the effective stress $\left(\tau_{\infty}\right.$ $-\tau_{f}$ ) and hence yields a good estimate of the strain energy change when used in the usual formula for an isolated fault.

For convenience of analysis the fault plane has been idealized as consisting of regions which slip freely at a uniform residual value of the friction stress and of regions which slip only at the time of an earthquake. In actuality, there is likely to be a distribution of strength on the fault plane and a relatively continuous variation of the slipping regions as the tectonic stress is increased. Of course, the interactions in this case will be much more complicated than those described here. Nevertheless, the idealization considered here is a limiting one and hence can reasonably be considered to yield bounds for intermediate situations. Moreover, there is evidence from direct observation of fault surfaces associated with seismic events in mines [McGarr et al., 1979a] that fault slip zones, at least in some instances, may not be planar. Again the analysis of this case is more complicated than that presented here, but some preliminary results have been obtained by Segall and Pollard [1980] and by McGarr et al. [1979b]. Three-dimensional effects may also be important, and Madariaga has given some general results which are applicable in this case. Although for the three-dimensional case there appears to be no simple calculation analogous to that presented here, McGarr [1981] has recently considered an approximate solution for an annular fault model. His predictions are comparable to those given here if the results are compared on the basis of fault area. More generally, the development of a fault plane may be a late stage in a process of inelastic deformation in a large re- 
gion. In this case an inclusion model like that suggested by Rudnicki [1977] may be appropriate, and the development of a fault may occur by the localization processes which were analyzed by Rudnicki and Rice [1975]. Indeed, Rice [1978] has suggested that the observations of Lindh et al. [1978] on the orientation of foreshocks relative to mainshock are consistent with this latter analysis. However, this misorientation may be related to the principal stress axes rotation caused by the foreshocks themselves [Rudnicki, 1979].

This analysis has also assumed that failure results in a reduction of the stress to the residual friction value. In general, the stress may not be reduced to this value upon failure, and consequently the friction stress on the fault plane will not be uniform. A limiting case is the model of Das and Aki [1977a, $b$ ] in which a propagating fault can leave unbroken ligaments behind its tip. The calculations given here for the barrier model are relevant to this case, but they are purely kinematic in nature, and for a more detailed analysis it would be necessary to introduce a fracture criteria. Moreover, for fault lengths which are comparable to the distance to the free surface or boundary of the seismogenic region, the details of propagation are likely to be affected by whether the boundary conditions are idealized as prescribed displacements or stresses [Freund, 1979].

In spite of all the limitations of the present analysis, it is useful in demonstrating the magnitude of the effects that interacting slip zones may have in fault processes.

\section{APPENDIX}

The relative displacement for the two collinear slip zones (Figure $4 b$ ) can be shown from expressions given by Erdogan [1962] to be

$$
\delta(x)=2(1-\nu) \tau \mu^{-1} \int_{x}^{b} \frac{\left(s^{2}-b^{2} \lambda^{2}\right) d s}{\left[\left(s^{2}-a^{2}\right)\left(b^{2}-s^{2}\right)\right]^{1 / 2}}
$$

where $\lambda^{2}=E(m) / F(m), m^{2}=1-a^{2} / b^{2}$, and $E(m)$ and $F(m)$ are the complete elliptic integrals defined following (11). This integral can be expressed as

$$
\delta(x)=2(1-\nu) \tau \mu^{-1} b\left[E(\phi, m)-\lambda^{2} F(\phi, m)\right]
$$

where

$$
\begin{aligned}
& F(\phi, m)=\int_{0}^{\phi}\left(1-m^{2} \sin ^{2} \phi\right)^{-1 / 2} d \theta \\
& E(\phi, m)=\int_{0}^{\phi}\left(1-m^{2} \sin ^{2} \theta\right)^{1 / 2} d \theta
\end{aligned}
$$

are elliptic integrals of the first and second kinds, respectively [Abramowitz and Stegun, 1964], and

$$
\sin ^{2} \phi=m^{-2}\left[1-(x / b)^{2}\right]
$$

The average displacement is simply the integral of $\delta(x)$ divided by $b-a$. Evaluation of the integral can be accomplished by using the results of Tranter [1961] or of Lowengrub and Srisvastava [1968] for the corresponding tensile crack problem, and the final expression can be written in the form of (15).

Acknowledgments. Helpful discussions of one of us (J.W.R.) with J. R. Rice are gratefully acknowledged. This work was supported by U.S. Geological Survey contract 14-08-0001-19146 at the University of Illinois, by the Earth Sciences Section, National Science Foundation grants EAR78-11973 and EAR77-13641, and U.S. Geological Survey contract 14-08-0001-18371 at the Seismological Laboratory of the California Institute of Technology, and by the U.S. Geological Survey while J. W. Rudnicki was geophysicist at the U.S. Geological Survey Center for Earthquake Studies, Menlo Park, California, during June 1979. Division of Geological and Planetary Sciences, California Institute of Technology contribution 3425 .

\section{REFERENCES}

Abramowitz, M., and I. A. Stegun (Eds), Handbook of Mathematical Functions, Appl. Math. Ser. 55, National Bureau of Standards, Washington, D. C., 1964.

Aki, K., Characterization of barriers on an earthquake fault, $J$. Geophys. Res., 84, 6140-6148, 1979.

Barenblatt, G. I., Mathematical theory of equilibrium cracks in brittle fracture, Adv. Appl Mech., 7, 55-129, 1962.

Barton, N., A model study of rock joint deformation, Intl. J. Rock Mech. Min. Sci., 9, 579-602, 1972.

Barton, N., Review of a new strength criterion for rock joints, Eng. Geol., 7, 287-332, 1973.

Bowden, F. P., and D. Tabor, Friction: An Introduction to Tribology, 178 pp., Anchor Books, Garden City, N. Y., 1973.

Das, S., and K. Aki, A numerical study of two dimensional spontaneous rupture propagation, Geophys. J. R. Astron. Soc., 50, 643-668, $1977 a$.

Das, S., and K. Aki, Fault plane with barriers: A versatile earthquake model, J. Geophys. Res., 82, 5658-5670, $1977 b$.

Erdogan, F., On stress distribution in plates with collinear cuts under arbitrary loads, in Proceedings of the 4th U.S. National Congress on Applied Mechanics, Vol. 1, edited by R. M. Rosenberg, pp. 547-553, American Society of Mechanical Engineers, Berkeley, Calif., 1962.

Freund, L. B., The mechanics of dynamic shear crack propagation, $J$. Geophys. Res., 84, 2199-2209, 1979.

Imamura, A., Theoretical and Applied Seismology, 348 pp., Maruzen, Tokyo, 1937.

Irwin, G. R., Analysis of stresses and strains near the end of a crack transversing a plate, J. Appl. Mech., 24, 361-364, 1957.

Ishida, M., and H. Kanamori, The foreshock activity of the 1971 San Fernando, California earthquake, Bull. Seismol. Soc. Am., 68, 1265-1279, 1978.

Ishida, M., and $\mathrm{H}$. Kanamori, Temporal variation of seismicity and spectrum of small earthquakes preceding the 1952 Kern County, California earthquake, Bull. Seismol. Soc. Am., 70, 509-528, 1980.

Jaeger, J. C., and N. G. W. Cook, Fundamentals of Rock Mechanics, 2nd ed., 585 pp., Halsted, New York, 1976.

Jones, L. M., and P. Molnar, Some characteristics of foreshocks and their possible relationship to earthquake prediction and premonitory slip on faults, J. Geophys. Res., 84, 3496-3608, 1979.

Kanamori, $H$., The energy release in great earthquakes, $J$. Geophys. Res., 82, 2981-2987, 1977.

Kanamori, H., Use of seismic radiation to infer source parameters, Proceedings of Conference III, Fault Mechanics and Its Relation to Earthquake Prediction, Geol. Surv. Open File Rep. U.S., 78-380, 283-318, 1978.

Kanamori, H., and D. L. Anderson, Theoretical basis of some empirical relations in seismology, Bull. Seismol. Soc. Am., 65, 1073-1095, 1975.

Kanamori, H., and G. S. Stewart, Seismological aspects of the Guatemala earthquake of February 4, 1976, J. Geophys. Res., 83, 3427$3434,1978$.

Knott, J. F., Fundamentals of Fracture Mechanics, 273 pp., Halsted, New York, 1973.

Koiter, W. T., An infinite row of collinear cracks in an infinite elastic sheet, Ing. Arch., 28, 168-172, 1959.

Kostrov, B. V., Seismic moment, energy of earthquakes, and seismic flow of rock, Izv. Acad. Sci. USSR Phys. Solid Earth, I, 23-40, 1974.

Lay, T., and H. Kanamori, Earthquake doublets in the Solomon Islands, Phys. Earth Planet. Inter., 2I, 283-304, 1980.

Lindh, A., G. Fuis, and C. Mantis, Seismic amplitude measurements suggest foreshocks have different focal mechanisms than aftershocks, Science, 201, 56-58, 1978.

Love, A. E. H., A Treatise on the Mathematical Theory of Elasticity, Dover, New York, 643 pp., 1927. (Reprint of 4th ed., Cambridge University Press, New York, 1944.) 
Lowengrub, M., and K. N. Srivastava, On two planar Griffith cracks in an infinite elastic solid, Intl. J. Eng. Sci., 6, 349-362, 1968.

Madariaga, R., Implications of stress-drop models of earthquakes for the inversion of stress drop from seismic observations, Pure Appl. Geophys., 115, 301-316, 1977.

Madariaga, $\mathbf{R}$., On the relation between seismic moment and stress drop in the presence of stress and strength heterogeneity, $J$. Geophys. Res., 84, 2243-2250, 1979.

McGarr, A., Analysis of peak ground motion in terms of a model of inhomogeneous faulting, $J$. Geophys. Res., in press, 1981.

McGarr, A., S. M. Spottiswoode, N. C. Gay, and W. D. Ortlepp, Observations relevant to seismic driving stress, stress drop, and efficiency, J. Geophys. Res., 84, 2251-2263, 1979a.

McGarr, A., D. Pollard, N. C. Gay, and W. D. Ortlepp, Observations and analysis of structures in exhumed mine-induced faults, Proceedings of Conference VIII, Analysis of Actual Fault Zones in Bedrock, Geol. Surv. Open File Rep. U.S., 79-1239, 101-120, 1979 b.

Mikumo, T., and T. Miyatake, Earthquake sequences on a frictional fault model with non-uniform strengths and relaxation times, Geophys. J. R. Astron. Soc., 59, 497-522, 1979.

Miyamura, S., S. Omote, R. Teisseyre, and E. Vesanen, Multiple shocks and earthquakes series pattern, Bull. Intl. Inst. Seismol. Earthquake Eng., 2, 71-92, 1964.

Muskhelishvili, N. I., Some Bosic Problems of the Mathematical Theory of Elasticity, translated from Russian by J. R. M. Radok, Noordhoff, Groningen, Netherlands, 1953.

Nagamune, T., Source regions of great earthquakes, Geophys. Mag., $35,333-399,1971$

Orowan, E., Mechanism of seismic faulting, Mem. Geol. Soc. Am., 79, $323-345,1960$

Rial, J. A., The Caracus, Venezuela, earthquake of July, 1962: multiple-source event, J. Geophys. Res., 83, 5405-5414, 1978.

Rice, J. R., An examination of the fracture mechanics energy balance from the point-of-view of continuum mechanics, in Proceedings of the 1st International Conference on Fracture, vol. I, edited by. T. Yokobori, pp. 283-308, Japanese Society for Strength and Fracture, Sendai, Japan, 1966

Rice, J. R., Mathematical analysis in the mechanics of fracture, in Fracture: An Advanced Treatise, vol. 2, eidted by H. Liebowitz, pp. 191-311, Academic, New York, 1968.
Rice, J. R., Models for the inception of earthquake rupture, Eos Trans. $A G U, 59,1204,1978$.

Rice, J. R., Theory of precursory processes in the inception of earthquake rupture, Gerlands Beitr. Geophys., 88, 91-127, 1979a.

Rice, J. R., The mechanics of earthquake rupture, in Proceedings of the International School of Physics, 'Enrico Fermi,' Italian Physical Society, Course LXX VIII on Physics of the Earth's Interior, edited by E. Boschi, North Holland, Amsterdam, in press, $1979 b$.

Richter, C. F., Elementary Seismology, 768 pp., W. F. Freeman, San Francisco, Calif., 1958.

Rudnicki, J. W., The inception of faulting in a rock mass with a weakened zone, J. Geophys. Res., 82, 844-854, 1977.

Rudnicki, J. W., The stabilization of slip on a narrow weakening fault zone by coupled deformation-pore fluid diffusion, Bull. Seismol. Soc. Am., 69, 1011-1026, 1979a.

Rudnicki, J. W., Rotation of principai stress axes caused by faulting, Geophys. Res. Lett., 6, 135-138, $1979 b$.

Rudnicki, J. W., Fracture mechanics applied to the earth's crust, Annu. Rev. Earth Planet Sci., 8, 489-525, 1980.

Rudnicki, J. W., and J. R. Rice, Conditions for the localization of deformation in pressure-sensitive dilatant materials, J. Mech. Phys. Solids, 23, 371-394, 1975.

Segall, P., and D. D. Pollard, Mechanics of discontinuous faults, $J$. Geophys. Res., 85, 4337, 1980.

Tada, H., P. C. Paris, G. R. Irwin, The Stress Analysis of Cracks Handhook, Del Research Corporation, Hellertown, Pa., 1973.

Tranter, C. J., The opening of a pair of coplanar Griffith cracks under internal pressure, Q. J. Mech. Appl. Math., 14, 283-292, 1961.

Trifunac, M. D., and J. N. Brune, Complexity of energy release during Imperial Valley, California earthquake of 1940, Bull. Seismol. Soc. Am., 60, 137-160, 1970.

Willmore, T. J., The distribution of stress in the neighborhond of a crack, Q. J. Mech. Appl. Math., 2, 53-63, 1949.

Wyss, M., and J. N. Brune, The Alaska earthquake of 28 March, 1964: A complex multiple rupture, Bull. Seismol. Soc. Am., 57, $1017-1023,1967$

(Received March 7, 1980;

revised September 2, 1980;

accepted September 22, 1980.) 
Appendix IV

On Energy Radiation from Seismic Sources

by

J. W. Rudnicki ${ }^{+}$and L. B. Freund ${ }^{*}$

Bulletin of the Seismological Society of America, 71, 583-596, 1981

+Department of Theoretical and Applied Mechanics, University of Illinois, Urbana, IL 61801

*

*Division of Engineering, Brown University, Providence, RI 02912 
Abstract

This note clarifies the relationships among various expressions for the energy radiated by elastodynamic seismic sources. The radiated energy can be expressed in terms of the far-field particle velocities provided that the stress-particle velocity relationship asymptotically approaches the plane wave relationship with increasing distance from the source. This condition is satisfied for all sources that can be characterized by a moment density tensor. For the case in which the source can be characterized as a point, i.e., the wave lengths of all emitted radiation are much greater than source dimensions, the radiated energy is expressed in terms of the moment tensor. The relation between these far-field representations and Kostrov's (1974) representation of radiated energy in terms of fault surface traction and particle velocity is established. Kostrov's representation is arranged in various forms to reveal the source of radiated energy as the deviations of the fault surface tractions and particle velocities from the values which would occur during quasistatic fault motion between the same end states. Moreover, the excess of the static strain energy change over the work done by the fault surface tractions, called $W_{0}$ by Kanamori (1977), is shown to be a good approximation to the radiated energy when fault propagation speed is near the Rayleigh wave velocity and the time rate of change of fault surface traction is small. 


\section{Introduction}

The determination of the energy released by an earthquake is one of the fundamental problems of seismology. Typically, empirical estimates of energy changes associated with earthquakes are obtained in two ways (Bath, 1966): from the transient dynamic displacements inferred from examination of seismograms or from measurements of the static deformation in the epicentral region. The first method yields an estimate of the seismic wave energy radiated to the farfield whereas the second yields an estimate of the energy which has gone into overall deformation of the region around the source. Although the energy determined by the second method is frequently used as an approximation to that determined by the first, these energies are not, in general, equal and the relationship between them is often unclear.

The purpose of this note is to present various expressions for the radiated energy in order to illuminate its origin in seismic faulting and to clarify the relationship of radiated energy to other energies involved in earthquake faulting. The treatment is, however, limited to infinite linear elastic solids which are isotropic and homogeneous. Although such conditions are, of course, not satisfied in the earth, this idealization has nevertheless proven to be useful both as an approximation and in providing guidance in more realistic problems. 


\section{Definition of Radiated Energy}

The radiated energy is defined here as

$$
E_{R}=\int_{-\infty}^{\infty} \int_{S}\left[-\sigma_{i j} \gamma_{j} \dot{u}_{i}\right] d S d t
$$

where $u_{i}$ is the change in displacement and $\sigma_{i j}$ is the change in stress associated with $u_{i}$. The remaining symbols in (1) are defined as follows: $t$ is time, the superposed dot denotes time differentiation, $S$ is a spherical surface of radius $r$ centered at the source with $r>l$, where $l$ is a characteristic source dimension, and $\gamma_{i}$ is the unit normal to $S$ directed outward from the source. The integrand of (1) is simply the rate of work by the material inside $S$ on the material outside $S$ and this agrees with the fundamental definition of energy flow. If the stress change $\sigma_{i j}$ and the particle velocity $\dot{\mathrm{u}}_{i}$ are proportional to $\mathrm{r}^{-1}$ as $r$ goes to infinity in any fixed direction, then $E_{R}$ has a finite nonzero value in this limit.

An expression for radiated energy which is frequently used in seismology (e.g., Haske11, 1964) is

$$
E_{R}=\int_{-\infty}^{\infty} \int_{S} \rho\left[c_{d}\left(\dot{u}_{i} \gamma_{i}\right)^{2}+c_{S}\left(\dot{u}_{i} m_{i}\right)^{2}\right] d S d t
$$

where $\rho$ is the density, $c_{d}$ and $c_{s}$ are the dilatational and shear wave speeds, respectively, and $\mathrm{m}_{i}$ is the unit tangent vector to $S$ in the direction of the shear traction. The definition (2) follows from (1) if

$$
\dot{\mathrm{u}}_{i}=0\left(\mathrm{r}^{-1}\right)
$$

and

$$
\sigma_{i j} \gamma_{j}+\rho \dot{u}_{j} \gamma_{j} \gamma_{i} c_{d}+\rho \dot{u}_{j} m_{j} m_{i} c_{s}=0\left(r^{-2}\right)
$$

as $r \rightarrow \infty$ in any fixed direction: Because the area of $S$ is proportional 
to $r^{2}$, terms which decay faster than $r^{-2}$ do not contribute to (1) in the limit of $r \rightarrow \infty$. The condition (4) implies that far from the source waves become asymptotic to plane waves in the sense that the traction and particle velocity are related as they are for plane waves or, in other words, that near the wavefront the curvature of the wave front is negligible. The equivalence of (1) and (2) has been discussed for the special cases of harmonic plane waves (Bullen, 1963; Sagisaka, 1945) and spherical waves (Yoshiyama, 1963) and it has been demonstrated by direct calculation for a double couple source (e.g., Dahlen, 1974), However, in the next section, it will be shown that (4) is satisfied, and hence (1) and (2) are equivalent, for any source which can be characterized by a moment density tensor. 


\section{Far-Field Representation}

The usual approach of source theory (e.g., Archambeau, 1968; Backus and Mulcahy, 1976) is to model inelastic processes due to faulting, explosions, etc. as causing a region of an elastic body (the source) to undergo a stressfree change of size and shape without altering the elastic properties of the region. If this change of size and shape is expressed as a strain $\varepsilon_{i j}^{T}(\underset{\sim}{x}, t)$ then the seismic moment density tensor is defined by (e.g., Kostrov, 1970; Aki and Richards, 1980, p. 59)

$$
\mathrm{m}_{i j}(\underset{\sim}{\mathrm{x}}, t)=\mathrm{C}_{i j k \ell} \varepsilon_{\mathrm{kl}}^{\mathrm{T}}(\underset{\sim}{\mathrm{x}}, t)
$$

where $C_{i j k l}$ is the tensor of elastic moduli. For an isotropic elastic solid the modulus tensor is given by

$$
C_{i j k \ell}=\mu\left(\delta_{i k} \delta_{j \ell}+\delta_{i \ell} \delta_{j k}\right)+\Lambda \delta_{i j} \delta_{k \ell}
$$

where $\mu$ is the shear modulus and $\Lambda$ is the Lame constant.

The displacements due to a given $m_{i j}(\underset{\sim}{x}, t)$ are then calculated as those due to an effective body force

$$
f_{i}^{e f f}=-\partial m_{j i} / \partial x_{j}
$$

The displacement field can be written as the sum

$$
\left.u_{i}(\underset{\sim}{x}, t)=u_{i}^{d}(\underset{\sim}{x}, t)+u_{i}^{s} \underset{\sim}{x}, t\right)
$$

where $u_{i}^{d}(\underset{\sim}{x}, t)$ and $\left.u_{i}^{s} \underset{\sim}{x}, t\right)$ are the separate contributions from dilatational and shear waves, respectively. The far-field representations of $u_{i}^{d}(\underset{\sim}{x}, t)$ and $u_{i}^{s}\left(x_{\sim}, t\right)$ are as follows: 


$$
\begin{aligned}
& u_{i}^{d}=\frac{1}{4 \pi \rho r c_{d}^{3}} \gamma_{i} \gamma_{j} \gamma_{k} \int_{v} \dot{m}_{j k}\left(\underset{\sim}{\xi}, t-r / c_{d}+\underset{\sim}{\gamma} \cdot \underset{\sim}{\xi} / c_{d}\right) d^{3} \underset{\sim}{\xi} \\
& u_{i}^{s}=\frac{1}{4 \pi \rho r c_{s}^{3}}\left[\frac{1}{2}\left(\delta_{i j} \gamma_{k}+\delta_{i k} \gamma_{j}\right)-\gamma_{i} \gamma_{j} \gamma_{k}\right] \\
& \mathrm{x} \int_{\mathrm{v}} \dot{\mathrm{m}}_{j k}\left(\underline{\xi}, \mathrm{t}-\mathrm{r} / \mathrm{c}_{\mathrm{s}}+\underset{\sim}{\gamma} \cdot \underset{\xi}{\xi} / \mathrm{c}_{\mathrm{s}}\right) \mathrm{d}^{3} \underset{\xi}{\xi}
\end{aligned}
$$

where $r$ is the distance from a point in the source region to the observation point, and the integral is over the volume of the source region. The expressions (9) are accurate to terms of order $\ell / r$ and $\lambda / \mathrm{r}$ where $\ell$ is a characteristic source dimension and $\lambda=2 \pi \mathrm{c} / \omega$ is the wavelength of the emitted radiation associated with the frequency $\omega$.

It is apparent from (9) that the particle velocity $\dot{u}_{i}$ decays as $r^{-1}$ and, consequently that (3) is satisfied. To verify (4) it is only necessary to show that the left hand side of (4) vanishes when evaluated from displacements given by (9) since only terms of order $r^{-1}$ have been included in (9). The stresses corresponding to (9) are, to the same order of approximation,

$$
\begin{aligned}
\sigma_{i j}^{d}= & -\gamma_{k} \gamma_{\ell}\left[2 \mu \gamma_{i} \gamma_{j}+\Lambda \delta_{i j}\right] I_{k \ell}\left(t, r ; c_{d}\right) \\
\sigma_{i j}^{s}= & -\mu I_{k \ell}\left(t, r ; c_{s}\right)\left[\frac{1}{2} \gamma_{i}\left(\gamma_{k} \delta_{\ell j}+\gamma_{\ell} \delta_{k j}\right)\right. \\
& \left.+\frac{1}{2} \gamma_{j}\left(\gamma_{k} \delta_{\ell i}+\gamma_{\ell} \delta_{k i}\right)-2 \gamma_{i} \gamma_{j} \gamma_{k} \gamma_{\ell}\right]
\end{aligned}
$$

where the contributions from $u_{i}^{d}$ and $u_{i}^{s}$ have been given separately, and

$$
\left.I_{k l}(t, r ; c)=\frac{1}{4 \pi \rho r c^{4}} \int_{v} \underset{m_{k l}}{.} \underset{\sim}{\xi}, t-r / c+\underset{\sim}{\gamma} \cdot \underset{\sim}{\xi} / c\right) d^{3} \underset{\sim}{\xi} .
$$


Substitution into the left-hand side of (4) yields

$$
\begin{aligned}
& {\left[\rho c_{d}^{2}-(2 \mu+\Lambda)\right] \gamma_{i} \gamma_{k} \gamma_{l} I_{k \ell}\left(t, r ; c_{d}\right)} \\
& \quad+\left[\rho c_{s}^{2} m_{i} m_{\ell}-\mu\left(\delta_{l i}-\gamma_{\ell} \gamma_{i}\right)\right] \gamma_{k} I_{k \ell}\left(t, r ; c_{s}\right)
\end{aligned}
$$

where the symmetry of $I_{k l}$. (due to the symmetry of $m_{k \ell}(\underset{\sim}{x}, t)$ ) has been used. From the definition of $c_{d}=[(2 \mu+\Lambda) / \rho]^{1 / 2}$, it is immediately clear that the first term, due to the contribution of $u_{i}^{d}$ vanishes. That the second vanishes as well can be seen from the definition of $c_{s}=(\mu / \rho)^{1 / 2}$ and by noting that $Q_{\ell}=\gamma_{k} I_{k \ell}$ is a vector with a component in the direction of $\underset{\sim}{m}$ that is given by

$$
\underset{\sim}{\mathrm{m}}(\underset{\sim}{\mathrm{m}} \cdot \underset{\sim}{Q})=\underset{\sim}{Q}-\underset{\sim}{\gamma}(\underset{\sim}{\gamma} \cdot \underset{\sim}{Q})
$$

where $\underset{\sim}{\gamma} \cdot \underset{\sim}{Q}=Q_{p} \gamma_{p} \cdot$ The component of $\underset{\sim}{Q}$ orthogonal to both $\underset{\sim}{m}$ and $\underset{\sim}{\gamma}$ vanishes because there is zero shear traction on $S$ in this direction. Thus, the terms of order $r^{-1}$ in the left hand side of (4) vanish and consequently (2) is a precise expression for the radiated energy in the limit that the surface $S$ recedes to infinity. The radiated energy can be expressed as

$$
\begin{aligned}
E_{R} & =\frac{1}{16 \pi^{2} \rho c_{d}^{5}} \int_{-\infty}^{\infty} \int_{S}\left\{\frac{\gamma_{j} \gamma_{k}}{r} \int_{v} \ddot{m}_{j k}\left(\underset{\sim}{\xi}, t-r / c_{d}+\underset{\sim}{\gamma} \cdot \underset{\sim}{\xi} / c_{d}\right) d^{3} \underset{\sim}{\xi}\right\}^{2} d S d t \\
& +\frac{1}{16 \pi^{2} \rho c_{s}^{5}} \int_{-\infty}^{\infty} \int_{S}\left\{\frac{1}{2} \frac{\left(m_{j} \gamma_{k}+m_{k} \gamma_{j}\right)}{r} \int_{v} \ddot{m}_{j k}\left(\underset{\sim}{\xi}, t-r / c_{s}+\underset{\sim}{\gamma} \cdot \underset{\sim}{\xi} / c_{s}\right) d^{3} \underset{\sim}{\xi}\right\}^{2} d S d t
\end{aligned}
$$

Also, if the Fourier transform of a function $g(t)$ is defined by

$$
\tilde{g}(\omega)=\int_{-\infty}^{\infty} g(t) e^{-i \omega t} d t,
$$

then the Parseval identity

$$
\int_{-\infty}^{\infty}|\tilde{g}(\omega)|^{2} d \omega=2 \pi \int_{-\infty}^{\infty}[g(t)]^{2} d t
$$


can be used to express $E_{R}$ in terms of integrals of the spectral density of $\mathrm{m}_{\mathrm{jk}}$ - (Boatright (1980) has recently used this representation to advantage in a study of spectral theory for circular seismic sources). It should be emphasized that the equivalence of (1) and (2) does not require that the source be a "point source", that is, that the dimensions of the source be much smaller than the wavelengths of any waves emitted by the source $(\ell<\lambda)$. Rather, it is required only that the distance between $S$ and the source be large compared to source dimensions and wavelengths $(r>l, r>\lambda)$. The operations indicated by (2) seem possible to perform observationa11y (assuming, of course, that propagation effects due to departure of earth from a homogeneous elastic medium can be handled and neglecting effects due to finite boundaries): particle velocities can be measured in the far field and integrated to get $E_{R}$. However, as demonstrated by Kostrov (1968) (also see Rice, 1980) in the case of slip on a planar fault, the complete spatial variation of slip over the fault plane cannot be resolved from far-field observations. Evidently, the shorter wavelength information necessary to resolve completely the fault slip and not transmitted to the farfield does not contribute to $E_{R}$. Nevertheless, it is not possible to express the radiated energy in terms of the seismic moment tensor, which is defined by

$$
M_{i j}(t)=\int_{V} m_{i j}(\xi, t) d^{3} \xi
$$

If, however, attention is restricted to wavelengths which are large by comparison to source dimensions (i.e., sufficiently low frequencies) the source is perceived in the far-field as a point and the radiated energy can be expressed in terms of $M_{i j}$. 


\section{Point Source Representation}

As shown by Kostrov (1970), the point source representation corresponds to neglecting the time differences $\underset{\sim}{\gamma} \cdot \underset{\sim}{\xi} / c$ between different points in the source region. In this case the integrals in (9) simply define the time derivative of the seismic moment tensor (17) and (9) can be rewritten as

$$
\begin{aligned}
& u_{i}^{d}=\frac{1}{4 \pi \rho c_{d}^{3} r} \gamma_{i} \gamma_{k} \gamma_{l} \dot{M}_{k \ell}\left(t-r / c_{d}\right) \\
& u_{i}^{s}=\frac{1}{4 \pi \rho c_{s}^{3} r}\left[\frac{1}{2}\left(\gamma_{\ell} \delta_{k i}+\gamma_{k} \delta_{l i}\right)-\gamma_{i} \gamma_{k} \gamma_{l}\right] \dot{M_{k l}}\left(t-r / c_{s}\right)
\end{aligned}
$$

Substituting these expressions into (2) yields

$$
\begin{aligned}
E_{R} & =\frac{1}{16 \pi^{2} \rho c_{s}^{5}} \int_{-\infty}^{\infty} \int_{\Omega}\left\{\left(\frac{c_{s}}{c_{d}}\right)^{5}\left[\gamma_{k} \gamma_{\ell} \ddot{M}_{k \ell}\left(t-r / c_{d}\right)\right]^{2}\right. \\
& \left.+\left[\frac{1}{2}\left(\gamma_{k} m_{\ell}+\gamma_{\ell} m_{k}\right) \ddot{M}_{k \ell}\left(t-r / c_{s}\right)\right]^{2}\right\} d \Omega d t
\end{aligned}
$$

where dS has been replaced by $r^{2} d \Omega$. The second square bracket can be rewritten by using the relation

$$
\frac{1}{2}\left(\gamma_{k} m_{\ell}+m_{k} \gamma_{\ell}\right) \ddot{M}_{k \ell}=\left[\left(\gamma_{k} \ddot{M}_{k \ell}\right)\left(\gamma_{p} \ddot{M}_{p l}\right)-\left(\gamma_{k} \gamma_{\ell} \ddot{M}_{k \ell}\right)^{2}\right]^{\frac{1}{2}}
$$

which follows from (13) by using the symmetry of $\ddot{M}_{k \ell}$, setting $Q=\underset{\sim}{\gamma} \cdot \underset{\sim}{M}$, and taking the magnitude of both sides. Using (20) in (19) yields

$$
\begin{aligned}
E_{R} & =\frac{1}{16 \pi^{2} \rho c_{s}^{5}} \int_{-\infty}^{\infty} \int\left(\frac{c s}{c_{d}}\right)^{5}\left[\gamma_{k} \gamma_{\ell} \ddot{M}_{k \ell}\left(t-r / c_{d}\right)\right]^{2} \\
& \left.+\gamma_{k} \gamma_{\ell} \ddot{M}_{k p}\left(t-r / c_{s}\right) \ddot{M}_{\ell p}\left(t-r / c_{s}\right)-\left[\gamma_{k} \gamma_{\ell} \ddot{M}_{k \ell}\left(t-r / c_{s}\right)\right]^{2}\right\} d \Omega d t
\end{aligned}
$$


Because the arguments of $\mathrm{M}_{\mathrm{k} \ell}$ depend only on time for a fixed radius $r$, the integrals over the spherical surface involve only the components of the unit normal. These integrals have the form

$$
I_{i j k \ell}=\int_{\Omega} \gamma_{i} \gamma_{j} \gamma_{k} \gamma_{\ell} d \Omega
$$

The integration is most easily accomplished by recognizing that the result must be an isotropic tensor and, thus, must comprise sums and products of Kronecker delta's:

$$
I_{i j k \ell}=c_{1} \delta_{i j} \delta_{k \ell}+c_{2} \delta_{i k} \delta_{j l}+c_{3} \delta_{i \ell} \delta_{j k}
$$

where $c_{1}, c_{2}, c_{3}$ are constants. Furthermore, because (22) is symmetric with respect to interchange of any two indices, $c_{1}=c_{2}=c_{3}=c$. The constant $c$ can be determined by integration for a particular component, say $I_{1122}=c$ and the result is

$$
I_{i j k \ell}=\frac{4 \pi}{15}\left(\delta_{i j} \delta_{k \ell}+\delta_{i k} \delta_{j \ell}+\delta_{i \ell} \delta_{j k}\right)
$$

Using this result and the corollary,

$$
I_{i j k k}=\int \gamma_{i} \gamma_{j} d \Omega=\frac{4}{3} \pi \delta_{i j}
$$

in (21) yields

$$
\begin{aligned}
& 60 \pi \rho c_{s}^{5} E_{R}=\left(\frac{c_{s}}{c_{d}}\right)^{5} \int_{-\infty}^{\infty}\left\{2 \ddot{M}_{i j}\left(t-r / c_{d}\right) \ddot{M}_{i j}\left(t-r / c_{d}\right)+\left[\ddot{M}_{k k}\left(t-r / c_{d}\right)\right]^{2}\right\} d t \\
& +\int_{-\infty}^{\infty}\left\{3 \ddot{M}_{i j}\left(t-r / c_{s}\right) \ddot{M}_{i j}\left(t-r / c_{s}\right)-\left[\ddot{M}_{k k}\left(t-r / c_{s}\right)\right]^{2}\right\} d t
\end{aligned}
$$

This expression can also be rewritten by introducing the deviatoric part of $M_{i j}$, that is, 


$$
M_{i j}^{\prime}=M_{i j}-\delta_{i j} M_{k k} / 3
$$

so that $\operatorname{tr} \underset{\sim}{\mathrm{M}^{\prime}}=\mathrm{M}_{\mathrm{kk}}^{\prime}=0$. Then $(26)$ becomes

$$
\begin{aligned}
60 \pi \rho c_{s}^{5} E_{R} & =\left(\frac{c_{s}}{c_{d}}\right)^{5} \int_{-\infty}^{\infty}\left\{2 \ddot{M}_{i j}^{\prime}\left(t-r / c_{d}\right) \ddot{M}_{i j}^{\prime}\left(t-r / c_{d}\right)+\frac{5}{3}\left[\ddot{M}_{k k}\left(t-r / c_{d}\right)\right]^{2}\right\} d t \\
& +3 \int_{-\infty}^{\infty} \ddot{M}_{i j}^{\prime}\left(t-r / c_{s}\right) \ddot{M}_{i j}^{\prime}\left(t-r / c_{s}\right) d t
\end{aligned}
$$

If the source disturbance is confined to a surface of displacement discontinuity, as is typically the case in modelling fault rupture, the seismic moment density tensor has the form (Burridge and Knopoff, 1964; Kostrov, 1970; Aki and Richards, 1980, p. 52)

$$
m_{i j}(\underset{\sim}{x}, t)=C_{i j k \ell} \underset{\sim}{(x)} n_{k}(\underset{\sim}{x}) \Delta u_{\ell} \underset{\sim}{(\underset{x}{x}, t)} \delta_{D}\left(S_{F}\right)
$$

where $\underset{\sim}{\mathrm{n}}$ is the local unit normal to the surface, $\Delta \mathbf{u}_{\ell}$ is the displacement discontinuity, and $\delta_{D}\left(S_{F}\right)$ is a Dirac singular function which converts an integral over a volume containing $\mathrm{S}_{\mathrm{F}}$ to an integral over the surface $\mathrm{S}_{\mathrm{F}}$. In a homogeneous, isotropic elastic solid the modulus tensor is given by (6) and, in this case, the moment density tensor is

$$
m_{i j}(\underset{\sim}{x}, t)=\left[\mu\left(n_{j} \Delta u_{i}+n_{i} \Delta u_{j}\right)+\Lambda n_{k} \Delta u_{k} \delta_{i j}\right] \delta_{D}\left(s_{F}\right)
$$

For shear faulting $n_{k} \Delta u_{k}=0$ and if the fault surface is assumed to be flat and to have a normal in the $x_{2}$ direction,

$$
\mathrm{m}_{i j}(\underset{\sim}{\mathrm{x}}, t)=\mu\left(\delta_{2 j} \Delta \mathbf{u}_{i}+\delta_{2 i} \Delta \mathbf{u}_{j}\right) \delta_{D}\left(S_{F}\right)
$$

The seismic moment (17) is

$$
M_{i j}(t)=\mu \int_{S_{F}}\left[\delta_{2 j} \Delta u_{i}\left(\xi_{1}, \xi_{3}, t\right)+\delta_{2 i} \Delta u_{j}\left(\xi_{1}, \xi_{3}, t\right)\right] d \xi_{1} d \xi_{3}
$$


If it is further assumed that slip is only in the $x_{1}$ direction, $\Delta \mathrm{u}_{i}=\Delta \mathrm{u} \delta_{i 1}$, and $\mathrm{M}_{12}=\mathrm{M}_{21}=\mathrm{M}$ are the only non-zero components of the seismic moment tensor:

$$
M(t)=\mu \int_{S_{F}} \Delta u\left(\xi_{1}, \xi_{3}, t\right) d \xi_{1} d \xi_{3}
$$

The expression for the energy radiated by a point source (28) then reduces to

$$
30 \pi \rho c_{s}^{5} E_{R}=2\left(\frac{c_{s}}{c_{d}}\right)^{5} \int_{-\infty}^{\infty} \ddot{M}^{2}\left(t-r / c_{d}\right) d t+3 \int_{-\infty}^{\infty} \ddot{M}^{2}\left(t-r / c_{s}\right) d t
$$

Alternatively, $E_{R}$ can be expressed in terms of the Fourier transform of $M$ by using (15) and (16). 


\section{Fault Surface Representation}

If the source can be idealized as a propagating crack, then, as shown by Kostrov (1974) the radiated energy can be expressed in terms of the fault surface traction and particle velocity. The resulting form for $E_{R}$ is useful for assessing the physical sources of radiated energy. Let $\mathrm{S}_{f}$ be a surface coinciding with the fault surfaces (excluding the extending edges), $\mathrm{S}_{\mathrm{o}}$ be a tube enclosing the crack edges and $\underset{\sim}{\mathrm{n}}$ be the unit normal directed into the material bounded by $\mathrm{S}, \mathrm{S}_{\mathrm{f}}$ and $\mathrm{S}_{\mathrm{o}}$ (Hence $\underset{\sim}{\mathrm{n}}=-\underset{\sim}{\gamma}$ on $\mathrm{S}$ and $\underset{\sim}{\mathbf{n}}$ is the outward normal to the crack surfaces on $\mathrm{S}_{f}$ ). A general power balance for this material requires that

$$
-\int_{S} \bar{\sigma}_{i j} n_{j} \dot{u}_{i} d S-\dot{K}-\dot{\bar{U}}=\bar{F}(t)+\int_{S_{F}} \bar{\sigma}_{i j} n_{j} \dot{u}_{i} d S
$$

where $\dot{\mathrm{K}}$ and $\dot{\overline{\mathrm{U}}}$ are the rates of increase of kinetic energy and strain energy, respectively; and $\bar{F}(t)$ is the time rate of energy flow to the extending fault edges. The stress $\bar{\sigma}_{i j}$ is the total stress, that is

$$
\bar{\sigma}_{i j}=\sigma_{i j}+\bar{\sigma}_{i j}^{\circ}
$$

and the total displacement is

$$
\bar{u}_{i}=u_{i}+\bar{u}_{i}^{\circ}
$$

with $\dot{\bar{u}}_{i}^{\circ}=0$. The left hand side of (35) is the excess of the total power input over the increase in total internal energy. The right hand side can be regarded as dissipation due to energy flux to the extending fault edges (first term) and dissipation due to the work of the fault surface tractions (second term). More precisely, the energy flux to the extending edges is defined as (Freund, 1972a; Kostrov, 1974)

$$
\bar{F}(t)=\lim _{S \rightarrow 0} \int_{S_{0}}\left\{\bar{\sigma}_{i j} n_{j} \dot{u}_{i}+\left[\frac{1}{2} \bar{\sigma}_{i j} \bar{u}_{i, j}+\frac{1}{2} \dot{u}_{i} \dot{u}_{i}\right] v\right\} d S
$$


where $v$ is the normal velocity of the fault edge in its own plane. The contribution from $\bar{F}(t)$ arises because the stress and particle velocity are singular at the edge of an extending crack in a linear elastic solid. Although this singularity is sometimes regarded as unrealistic, the energy flux can be given a simple physical interpretation as the negative of the work of unloading the fracture surfaces, or the energy which is needed to overcome the fracture resistance of the material just ahead of the fault edge (Freund, 1972a; Kostrov, 1974). Specific forms for $\bar{F}(t)$ have been given for semi-infinite cracks (or finite cracks prior to reflected waves) by Freund (1972b) for plane strain extension (Mode I), by Fossum and Freund (1975) for plane strain shear (Mode II) and by Atkinson and Eshelby (1968 for antiplane shear (Mode III). Because the initial stress is nonsingular at a point on the fault edge for any nonzero amount of fault expansion at that point, the energy flux into the edge depends only on the incremental stress and displacement, i.e., $\bar{F}(t)$ may be replaced by $F(t)$ in all cases.

Equation (35) can be rearranged by substituting (36) and using the definition of radiated energy (1). The result is

$$
-\dot{\mathrm{E}}_{\mathrm{R}}-\int_{S} \bar{\sigma}_{i j}^{0} \mathrm{n}_{j} \dot{\mathrm{u}}_{i} \mathrm{dS}-\dot{\mathrm{K}}-\dot{\overline{\mathrm{U}}}=\overline{\mathrm{F}}(\mathrm{t})+\int_{S_{F}} \bar{\sigma}_{i j} \mathrm{n}_{j} \dot{\mathrm{u}}_{i} \mathrm{dS}
$$

Integrating in time and recognizing that the kinetic energy vanishes in the initial and final static states yield the following expression:

$$
\begin{gathered}
E_{R}+\int_{S} \bar{\sigma}_{i j}^{0} n_{j} u_{i}^{f i n a l} d S+\left(\bar{U}_{f i n a l}-\bar{U}_{\text {initial }}\right) \\
=-\int_{-\infty}^{\infty} \bar{F}(t) d t-\int_{-\infty}^{\infty} \int_{S_{F}} \bar{\sigma}_{i j} n_{j} \dot{u}_{i} d S d t
\end{gathered}
$$

For quasistatic propagation $E_{R}=0$ and (37) reduces to a formula for the change in strain energy:

$$
\bar{U}_{\text {final }}-\bar{U}_{\text {initial }}+\int_{S} \bar{\sigma}_{i j}^{\circ} n_{j} u_{i}^{f i n a l} d S=-\int_{-\infty}^{\infty} \int_{S_{F}}\left[\bar{\sigma}_{i j} n_{j} \dot{u}_{i}\right]_{0} d S d t-\int_{-\infty}^{\infty} \bar{F}_{0}(t) d t
$$


where the subscript "o" denotes the value during quasistatic extension. The integral over $S$ is frequently neglected by assuming $S$ coincides with the earth's surface and, hence, is traction-free. However, this assumption is not needed to obtain the following expressions for radiated energy. Subtracting (38) from (37) yields

$E_{R}=\int_{-\infty}^{\infty} \int_{S_{F}}\left\{\left[n_{j} \bar{\sigma}_{i j} \dot{u}_{i}\right]_{0}-\cdot\left[n_{j} \bar{\sigma}_{i j} \dot{u}_{i}\right]\right\} d S d t+\int_{-\infty}^{\infty}\left\{\bar{F}_{0}(t)-\bar{F}(t)\right\} d t$

where the superposed bars can be omitted in this equation. The integrand of the second term is the difference in energy flux to the crack-tip during quasistatic and dynamic propagation of the fault edge and the integrand of the first term is the difference in the work rate of the fault surface tractions (behind the fault edge) during quasistatic and dynamic extension. If the fault surface tractions are bounded the first term of (39) can be integrated by parts. The result is

$E_{R}=\int_{-\infty}^{\infty} \int_{S_{F}}\left\{\left[n_{j} \dot{\bar{\sigma}}_{i j} u_{i}\right]-\left[n_{j} \dot{\bar{\sigma}}_{i j} u_{i}\right]_{0}\right\} d S d t+\int_{-\infty}^{\infty}\left[\bar{F}_{0}(t)-\bar{F}(t)\right] d t$

where the integrated terms vanish because the initial and final static states are fixed and the superposed bars can again be omitted. This expression is essentially that given by Kostrov (1974, eq. 2.26), although he does not write it in this way. This form for $E_{R}$ makes clear the role of the time-dependent surface tractions in radiating energy.

An alternative form for the radiated energy can be obtained by using (36) in the first term on the right hand side of (38). The result is $\int_{-\infty}^{\infty} \int_{S_{F}}\left[\bar{\sigma}_{i j} n_{j} \dot{u}_{i}\right]_{0} d S d t=\int_{-\infty}^{\infty} \int_{S_{F}}\left[\sigma_{i j} n_{j} \dot{u}_{i}\right]_{0} d S d t+\int_{S_{F}} \bar{\sigma}_{i j}^{0} n_{j} u_{i}^{f i n a l} d S$

If $\sigma_{i j}$ depends on time only through $u_{i}$, that is

$\left.\sigma_{i j}(\underset{\sim}{x}, t)=\sigma_{i j}\left[\underset{\sim}{x}, u_{i} \underset{\sim}{x}, t\right)\right]$, then 


$$
\int_{-\infty}^{\infty} \int_{S_{F}}\left[\sigma_{i j} n_{j} \dot{u}_{i}\right]_{0} d S d t=\int_{S_{F}}\left[\left(\sigma_{i j} n_{j}\right) \text { ave } u_{i}^{f i n a l}\right] d S
$$

where this equation can be used to define the average traction $\left(\sigma_{i j} n_{j}\right)$ ave In the special case where $\sigma_{i j} n_{j}$ is proportional to $u_{i}$

$$
\left(\sigma_{i j} n_{j}\right)_{\text {ave }}=\frac{1}{2}\left[\left(\sigma_{i j} n_{j}\right)_{f i n a l}+\left(\sigma_{i j} n_{j}\right)_{i n i t i a l}\right] \text {. }
$$

Note that $\left(\sigma_{i j} n_{j}\right)_{\text {initial }}$ is not the traction existing ahead of the extending fault edge, but rather that which exists on the fault surface just after the passage of the edge. Noting that

$$
\left(\sigma_{i j} n_{j}\right)_{\text {ave }}=\left(\bar{\sigma}_{i j} n_{j}\right)_{\text {ave }}-\bar{\sigma}_{i j}^{\circ} n_{j}
$$

and using (42) in (41) yield

$$
\int_{-\infty}^{\infty} \int_{F}\left[\bar{\sigma}_{i j} n_{j} \dot{u}_{i}\right]_{0} d S d t=\int_{-\infty}^{\infty} \int_{S_{F}}\left[\left(\bar{\sigma}_{i j} n_{j}\right)_{\text {ave }} \dot{u}_{i}\right]_{0} d S d t
$$

Consequently, (38) can be written as follows:

$\overline{\mathrm{U}}_{\text {final }}-\overline{\mathrm{U}}_{\text {initial }}+\int_{S} \bar{\sigma}_{i j}^{\circ} \mathrm{n}_{j} \mathrm{u}_{i}^{\mathrm{final}} \mathrm{dS}=\int_{-\infty}^{\infty} \int_{S_{F}}\left[\left(\bar{\sigma}_{i j} \mathrm{n}_{j}\right)_{\text {ave }} \dot{\mathrm{u}}_{i}\right]_{0} \mathrm{dS} \mathrm{dt}-\int_{-\infty}^{\infty} \overline{\mathrm{F}}_{0}(\mathrm{t}) \mathrm{dt}$

If $\overline{\mathrm{F}}_{\mathrm{O}}$ and the integral over $\mathrm{S}$ are assumed to be negligible, this equation reduces to the usual formula for strain energy change (e.g. Dahlen, 1974; Aki and Richards, 1980, p. 57). Subtracting (44) from (37) reveals that an alternative expression for the radiated energy is

$$
\left.E_{R}=\int_{-\infty}^{\infty} \int_{S_{F}}\left\{\left[\bar{\sigma}_{i j} n_{j}\right)_{\text {ave }} \dot{u}_{i}\right]_{0}-\left[\bar{\sigma}_{i j} n_{j} \dot{u}_{i}\right]\right\} d S d t+\int_{-\infty}^{\infty}\left\{\bar{F}_{0}(t)-\bar{F}(t)\right\} d t
$$

where again the superposed bars can be omitted.

If the fault surface tractions are time-independent (except possibly in a region near the fault edge which is small compared with the overall 
length), the first term of (39), (40) and (45) vanishes and the radiated energy is simply

$$
E_{R}=\int_{-\infty}^{\infty}\left[\bar{F}_{0}(t)-\bar{F}(t)\right] d t
$$

The expression for the radiated energy used by Husseini and Randa11 (1976) is a special case of (46). When the crack propagation speeds are near the limiting velocities (Rayleigh wave speed in plane strain or shear wave speed in antiplane strain), as is typical for observed earthquake faults, $\bar{F}(t)$ is small compared with $\bar{F}_{0}(t)$. Thus, in this case, the radiated energy is approximately equal to

$$
E_{R}=\int_{-\infty}^{\infty} \bar{F}_{0}(t) d t
$$

The meaning of this equation can be clarified by returning to (38) and recognizing that the first term on the right hand side is the work done by the fault surface tractions during quasistatic fault extension. In the simplest models of shear faults, these tractions are assumed to be equal to a uniform value of the friction stress and the corresponding work done is taken to be consumed in the generation of heat (e.g., Orowan, 1960). Thus, the right hand side of (47) is the excess of the strain energy change over the quasistatic work of the fault surface tractions at least if the integral over $S$ in (38) can be neglected. In the case of a shear fault with uniform stress drop, Kanamori (1977) has called this excess $W_{0}$ and has argued on an empirical basis that for large earthquakes $\mathrm{W}_{\mathrm{o}}$ is a good approximation to the radiated energy.

If the stress near the fault edge is not idealized as singular, the terms $\bar{F}(t)$ and $\bar{F}_{0}(t)$ vanish and the entire contribution to $E_{R}$ is from the first term of (39), (40) and (45). This is typically the case in 
dislocation models for which the rise time is finite (e.g., Haskell, 1964). Hence, the radiated energy is due to the deviation of the actual fault surface tractions during the dynamic event from the average fault plane tractions. It is interesting to note that for the configuration considered by Haskell (1964) (introduction of a rectangular dislocation loop of constant relative displacement) the strain energy change $U_{\text {final }}-U_{\text {initial }}$ is unbounded but the radiated energy (which Haskell computed by using (2)) is finite. Because $\bar{F}(t)$ and $\bar{\sigma}_{i j}^{0}$ are zero, the remaining term in (37) must also be unbounded. However, the sum of this term and strain energy change must be finite in order that $E_{R}$ is finite. 


\section{Concluding Discussion}

Various expressions for the energy radiated by seismic sources in linear elastic solids have been examined for the purpose of clarifying the meaning of radiated energy and its relation to seismic parameters. The term "earthquake energy" often seems to be applied either to $E_{R}$, the radiated energy, or to $W_{0}$, the excess of the strain energy change over the work done by the fault surface tractions during quasistatic fault extension (see (38) and (47)). However, these two quantities are equal only in special cases. As indicated by (40), $E_{R}$ depends on the time dependence of the fault surface tractions and particle velocities and, in particular, on the difference between the values during the actual dynamic process and those which would occur if the same fault extension occurred quasistatically. Hence, as pointed out by Kostrov (1974), the radiated energy cannot, in general, be determined from differences in the static end states. Nevertheless, $E_{R}$ can be determined by the measurement and integration of farfield particle velocities (2) even though this information is not sufficient to resolve the spatial distribution of fault slip. If, however, the fault surface tractions are assumed to be time independent, as in the case of many simple fault models, and the fault rupture speed is such that the second term in (46) is sma11, then $E_{R}$ can be approximated as in (47), where the righthand side of this equation is the quantity Kanamori (1977) has labelled $W_{0}$. This quantity does depend only on the difference between static end states.

Kanamori (1977) has argued that for large earthquakes $W_{0}$ is a good approximation to the energy computed from the Gutenberg-Richter energy magnitude relationship $(E=1.5 M+11.8$ where $M$ is the magnitude). More specifically, Kanamori (1977) showed that a magnitude computed using $W_{0}$ as the 
energy in the Gutenberg-Richter relation agrees well with the surface wave magnitude. The Gutenberg-Richter relationship (Gutenberg and Richter, 1942) was apparently established by estimating empirically the integral in (2) and hence the energy in this relation is an estimate for $E_{R}$. However, it is not clear whether Kanamori's (1977) results should be construed as evidence that the conditions for which (47) applies (time independent fault surface tractions and rupture speed near the limiting value) are satisfied for large earthquakes. Certainly, it seems unlikely that fault surface tractions would be time independent during faulting but it is possible that the contribution to the radiated energy is small.

Although the dislocation model of Haskell (1964) is often used as a basis for discussing energy radiation in earthquakes, it would seem to have some deficiencies in this regard. (Related deficiencies of the Haske11 model have been discussed by Madariaga (1978).) As mentioned earlier, the strain energy change for this configuration is infinite and hence the ratio of radiated energy to strain energy change is finite would be more useful for studying the relation between $W_{0}$ and $E_{R}$.

Acknowledgement

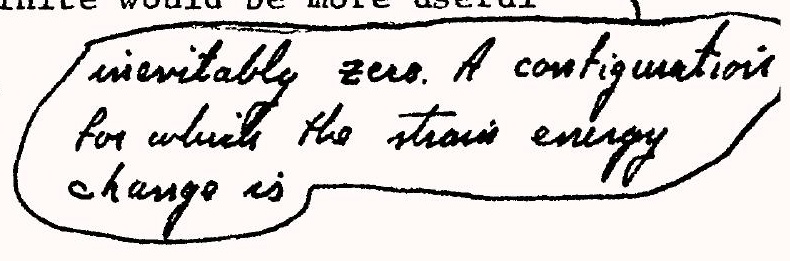

H. Kanamori and F. A. Dahlen read an earlier draft of the paper and provided helpful comments. J. R. Rice and P. G. Richards also made suggestions that improved the manuscript and independently pointed out the importance of distinguishing between incremental and total tractions in defining radiated energy. A portion of the work by J.W.R. was supported by the U.S. Geological Survey through contract GS 14-08-0001-19146 to the University of I1linois at Urbana. 


\section{$\underline{\text { References }}$}

Aki, K. and P. G. Richards, Quantitative Seismology: Theory and Methods, W. H. Freeman, San Francisco, 557 pp., 1980 .

Archambeau, C. B., General Theory of elastodynamic source fields, Rev. Geophys. Space Phys., $6,241-288,1968$.

Atkinson, C. and J. D. Eshelby, The flow of energy into the tip of a moving crack, J. Fracture Mech., 4, 3-8, 1968.

Backus, G. E. and M. Mulcahy, Moment tensors and other phenomenological descriptions of seismic sources - I. Continuous displacements, Geophys. J., 46, 341-361, 1976 .

Bath, M., Earthquake energy and magnitude, in Physics and Chemistry of the Earth, ed. by L. H. Ahrens, pp. 117-165, Pergamon Press, New York, 1966.

Boatwright, J., A spectral theory for circular seismic sources; simple estimates of source dimension, dynamic stress drop, and radiated seismic energy. Bul1. Seism. Soc. Am., 70, 1-27, 1980.

Bullen, K., An Introduction to the Theory of Seismology, 3rd Edition, Cambridge University Press, Cambridge, 1963.

Burridge, R. and L. Knopoff, Body force equivalents for seismic dislocations, Bull. Seism. Soc. Am., 54, 1875-1888, 1964.

Dahlen, F. A., On the ratio of $P$-wave to $S$-wave corner frequencies for shallow earthquake sources, Bul1. Seism. Soc. Am., 64, 1159-1180, 1974.

Dahlen, F. A., The balance of energy in earthquake faulting. Geophys. J. R. astr. Soc., 48, 239-261, 1977.

Freund, L. B., Energy flux into the tip of an extending crack in an elastic solid, J. of Elasticity, 2, 341-349, 1972a.

Freund, L. B., Crack propagation in an elastic solid subjected to general loading - I. Constant rate of extension, J. Mech. Phys. Solids, 20, 129-140, $1972 b$.

Fossum, A. F. and L. B. Freund, Nonuniform1y moving shear crack model of a shallow focus earthquake mechanism, J. Geophys. Res., 80, 3343-3347, 1975.

Gutenberg, B. and C. F. Richter, Earthquake magnitude, intensity, energy and acceleration, Bull. Seism. Soc. Am., 32, 163-191, 1942.

Haske11, N. A., Total energy and energy spectral density of elastic wave radiation from propagating faults. Bull. Seism. Soc. Am., 54, 1811-1841, 1964. 
Husseini, M. I. and M. J. Randall, Rupture velocity and radiation efficiency. Bull. Seism. Soc. Am., 66, 1173-1187, 1976.

Kanamori, H., The energy release in great earthquakes, J. Geophys. Res., 82 , 2981-2987, 1977.

Kostrov, B. V., The inverse problems of the theory of tectonic foci, Izv. Earth Physics, 9, 18-29, 1968.

Kostrov, B. V., The theory of the focus for tectonic earthquakes, Izv. Earth Physics, 4, 84-101, 1970.

Kostrov, B. V., Seismic moment and energy of earthquakes and seismic flow of rock. Izv. Earth Physics, 1, 23-40, 1974.

Madariaga, R., The dynamic field of Haskell's rectangular dislocation fault mode1, Bu11. Seism. Soc. Am., 68, 869-887, 1978.

Orowan, E., Mechanism of seismic faulting. Geol. Soc. Am. Mem., 79, 323-345, 1960 .

Rice, J. R., Mechanics of earthquake rupture. Proceedings of the International School of Physics, "Enrico Fermi", Italian Physical Society, Course LXX VIII (1979, Varenna on Lake Como, Italy) on Physics of the Earth's Interior, edited by E. Boshi, North Holland Publishing Company (publication expected 1980-1981) 1980.

Sagisaka, K., On the energy of earthquakes, Geophysical Magazine, 26, 53-82, 1954 .

Yoshiyama, R., Note on earthquake energy, Bulletin Earthquake Res. Inst., 41, $687-697,1963$. 